\title{
Stochastic processes and their spectral representations over non-archimedean fields
}

\author{
S.V. Ludkovsky
}

25.10 .2007

\begin{abstract}
The article is devoted to stochastic processes with values in finiteand infinite-dimensional vector spaces over infinite fields $\mathbf{K}$ of zero characteristics with non-trivial non-archimedean norms. For different types of stochastic processes controlled by measures with values in $\mathbf{K}$ and in complete topological vector spaces over $\mathbf{K}$ stochastic integrals are investigated. Vector valued measures and integrals in spaces over $\mathbf{K}$ are studied. Theorems about spectral decompositions of nonarchimedean stochastic processes are proved.
\end{abstract}

1

\section{Introduction}

Stochastic integrals and spectral representations of stochastic processes are widely used over the fields of real and complex numbers $[7,16,17,18,39$, 40]. If consider stochastic processes in topological groups or metric spaces it gives some generalization, but many specific features of topological vector spaces and results in them may naturally be missed $[35,34,18,40]$. At the same time non-archimedean analysis is being fast developed in recent years $[22,37,38,41,11]$. It has found applications in non-archimedean

\footnotetext{
${ }^{1}$ key words and phrases: stochastic processes, non-archimedean field, zero characteristic, random process, linear space, stochastic integral, spectral representation Mathematics Subject Classification 2000: 60G50, 60G51, 30G06
} 
quantum mechanics and quantum field theory $[41,3,9,6,19]$. These parts of mathematical physics heavily depend on probability theory [2]. Then it is very natural in the analysis on totally disconnected topological spaces and totally disconnected topological groups [37, 28]. Stochastic processes on such groups also permit to investigate their isometric representations in non-archimedean spaces.

Remind that non-archimedean fields $\mathbf{K}$ have non-archimedean norms, for example, for the field of $p$-adic numbers $\mathbf{Q}_{\mathbf{p}}$, where $p>1$ is a prime number $[22,37,42]$. Multiplicative norms in such fields $\mathbf{K}$ satisfy the strong triangle inequality: $|x+y| \leq \max (|x|,|y|)$ for each $x, y \in \mathbf{K}$.

Besides locally compact fields we consider also non locally compact fields. For example, the algebraic closure of $\mathbf{Q}_{\mathbf{p}}$ can be supplied with the multiplicative non-archimedean norm and its completion relative to this norm gives the field $\mathbf{C}_{\mathbf{p}}$ of complex $p$-adic numbers. The field $\mathbf{C}_{\mathbf{p}}$ is algebraically closed and complete relative to its norm [22]. Its valuation group $\Gamma_{\mathbf{C}_{\mathbf{p}}}:=\{|z|: z \in$ $\left.\mathbf{C}_{\mathbf{p}}, z \neq 0\right\}$ is isomorphic with the multiplicative group $\left\{p^{x}: x \in \mathbf{Q}\right\}$. There exist larger fields $\mathbf{U}_{\mathbf{p}}$ being extensions of $\mathbf{Q}_{\mathbf{p}}$ such that $\Gamma_{\mathbf{U}_{\mathbf{p}}}=\left\{p^{x}: x \in \mathbf{R}\right\}$. There are known extensions with the help of the spherical completions also, if an initial field is not such [37, 38, 8, 11].

Stochastic processes on spaces of functions with domains of definition in a non-archimedean linear space and with ranges in the field of real $\mathbf{R}$ or complex numbers $\mathbf{C}$ were considered in works [4, 12]-[15, 21, 23]. Another types of non-archimedean stochastic processes are possible depending on a domain of definition, a range of values of functions, values of measures in either the real field or a non-archimedean field [24, 30, 32, 43]. Moreover, a time parameter may be real or non-archimedean and so on, that is a lot of problems for investigations arise.

Stochastic processes with values in non-archimedean spaces appear while their studies for non-archimedean Banach spaces, totally disconnected topological groups and manifolds [25]-[29]. Very great importance branching processes in graphs also have $[1,17,18]$. For finite or infinite graphs with finite degrees of vertices there is possible to consider their embeddings into $p$-adic graphs, which can be embedded into locally compact fields. Considerations of such processes reduce to processes with values in the field $\mathbf{Q}_{\mathbf{p}}$ of $p$-adic numbers. Stochastic processes on $p$-adic graphs also have applications in analysis of flows of information, mathematical psychology and biology [20].

More specific features arise, when measures with values in non-archimedean 
fields are considered, so this article continuous previous works of the author in this area $[25,30,31,32]$.

In this article representations of stochastic processes with values in finiteand infinite-dimensional vector spaces over infinite fields with non-trivial non-archimedean norms are investigated. Below different types of stochastic processes controlled by measures with values in non-archimedean fields of zero characteristic and stochastic integrals are studied. Theorems about spectral decompositions of non-archimedean stochastic processes are proved (see, for example, $\S \S 20-29,75-82$, Lemmas 27 and 29, Theorems 20, 79, 81). Moreover, special features of the non-archimedean case are elucidated. These features arise from many differences of the classical over $\mathbf{R}$ and $\mathbf{C}$ analysis and the non-archimedean analysis. General constructions of the paper are illustrated in Examples 9, 31.1, 40, 74, Theorem 41, etc., where applications to totally disconnected topological groups are discussed as well.

Some necessary facts from non-archimedean probability theory and nonarchimedean analysis are recalled that to make reading easier (see, for example, $\S \S 1-6$ in section 2), as well as developed below, when it is essential. The main results of this paper are obtained for the first time. It is necessary to note that in this article measures and stochastic processes with values not only in non-archimedean fields (see section 2), but also with values in topological linear spaces which may be infinite dimensional over non-archimedean fields are studied (see $§ \S 44-74$ in section 3).

Stochastic processes with values in $\mathbf{Q}_{\mathbf{p}}^{\mathbf{n}}$ have natural interesting applications, for which a time parameter may be either real or $p$-adic. A random trajectory in $\mathbf{Q}_{\mathbf{p}}^{\mathbf{n}}$ may be continuous relative to the non-archimedean norm in $\mathbf{Q}_{\mathbf{p}}$, but its trajectory in $\mathbf{Q}^{\mathbf{n}}$ relative to the usual metric induced by the real metric may be discontinuous. This gives new approach to spasmodic or jump or discontinuous stochastic processes with values in $\mathbf{Q}^{\mathbf{n}}$, when the latter is considered as embedded into $\mathbf{R}^{\mathbf{n}}$.

\section{Scalar spectral functions}

To avoid misunderstandings we first present our notations and definitions and recall the basic facts.

1. Definitions. Let $G$ be a completely regular totally disconnected topological space, let also $\mathcal{R}$ be its covering ring of subsets in $G, \bigcup\{A: A \in$ 
$\mathcal{R}\}=G$. We call the ring separating, if for each two distinct points $x, y \in G$ there exists $A \in \mathcal{R}$ such that $x \in A, y \notin A$. A subfamily $\mathcal{S} \subset \mathcal{R}$ is called shrinking, if an intersection of each two elements from $\mathcal{A}$ contains an element from $\mathcal{A}$. If $\mathcal{A}$ is a shrinking family, $f: \mathcal{R} \rightarrow \mathbf{K}$, where $\mathbf{K}=\mathbf{R}$ or $\mathbf{K}$ is the field with the non-archimedean norm, then it is written $\lim _{A \in \mathcal{A}} f(A)=0$, if for each $\epsilon>0$ there exists $A_{0} \in \mathcal{A}$ such that $|f(A)|<\epsilon$ for each $A \in \mathcal{A}$ with $A \subset A_{0}$.

A measure $\mu: \mathcal{R} \rightarrow \mathbf{K}$ is a mapping with values in the field $\mathbf{K}$ of zero characteristic with the non-archimedean norm satisfying the following properties:

(i) $\mu$ is additive;

(ii) for each $A \in \mathcal{R}$ the set $\{\mu(B): B \in \mathcal{R}, A \subset B\}$ is bounded;

(iii) if $\mathcal{A}$ is the shrinking family in $\mathcal{R}$ and $\bigcap_{A \in \mathcal{A}} A=\emptyset$, then $\lim _{A \in \mathcal{A}} \mu(A)=$ 0 .

Measures on $\operatorname{Bco}(G)$ are called tight measure, where $\operatorname{Bco}(G)$ is the ring of clopen (simultaneously open and closed) subsets in $G$.

For each $A \in \mathcal{R}$ there is defined the norm: $\|A\|_{\mu}:=\sup \{|\mu(B)|: B \subset$ $A, B \in \mathcal{R}\}$. For functions $f: G \rightarrow X$, where $X$ is a Banach space over $\mathbf{K}$ and $\phi: G \rightarrow[0,+\infty)$ define the norm $\|f\|_{\phi}:=\sup \{|f(x)| \phi(x): x \in G\}$.

More generally for a complete locally $\mathbf{K}$-convex space $X$ with a family of non-archimedean semi-norms $\mathcal{S}=\{u\}$ [33] define the family of semi-norms $\|f\|_{\phi, u}:=\sup \{u(f(x)) \phi(x): x \in G\}$. Recall that a subset $V$ in $X$ is called absolutely $\mathbf{K}$-convex or a $\mathbf{K}$-disc, if $V B+V B \subseteq V$, where $B:=\{x \in \mathbf{K}$ : $|x| \leq 1\}$. Translates $x+V$ of absolutely $\mathbf{K}$-convex sets are called $\mathbf{K}$-convex, where $x \in X$. A topological vector space over $\mathbf{K}$ is called $\mathbf{K}$-convex, if it has a base of $\mathbf{K}$-convex neighborhoods of zero (see 5.202 and 5.203 [33]). A seminorm $u$ in $X$ is called non-archimedean, if $u(x+y) \leq \max [u(x), u(y)]$ for each $x, y \in X$. A topological vector space $X$ over $\mathbf{K}$ is locally $\mathbf{K}$-convex if and only if its topology is generated by a family of non-archimedean semi-norms. Therefore, a complete $\mathbf{K}$-convex space is the projective limit of Banach spaces over $\mathbf{K}$ (see 6.204, 6.205 and 12.202 [33]).

Put also $N_{\mu}(x):=\inf \left\{\|U\|_{\mu}: x \in U \in \mathcal{R}\right\}$ for each $x \in G$. If a function $f$ is a finite linear combination over the field $\mathbf{K}$ of characteristic functions $C h_{A}$ of subsets $A \subset G$ from $\mathcal{R}$, then it is called simple. A function $f: G \rightarrow X$ is called $\mu$-integrable, if there exists a sequence $f_{1}, f_{2}, \ldots$ of simple functions such that there exists $\lim _{n \rightarrow \infty}\left\|f-f_{n}\right\|_{N_{\mu}, u}=0$ for each $u \in \mathcal{S}$.

The space $L(\mu, X)=L(G, \mathcal{R}, \mu, X)$ of all $\mu$-integrable functions with 
values in $X$ is $\mathbf{K}$-linear. At the same time $\int_{G} \sum_{j=1}^{n} a_{j} C h_{A_{j}}(x) \mu(d x):=$ $\sum_{j=1}^{n} a_{j} \mu\left(A_{j}\right)$ for simple functions extends onto $L(\mu, X)$, where $a_{j} \in X$, $A_{j} \in \mathcal{R}$ for each $j$.

Put $\mathcal{R}_{\mu}:=\left\{A: A \subset G, C h_{A} \in L(\mu, \mathbf{K})\right\}$. For $A \in \mathcal{R}_{\mu}$ let $\bar{\mu}(A):=$ $\int_{G} \chi_{A}(x) \mu(d x)$.

For $1 \leq q<\infty$ denote by

$\|f\|_{q}:=\left[\sup _{x \in G}|f(x)|^{q} N_{\mu}(x)\right]^{1 / q}$ for a simple function $f: G \rightarrow X$, when $X$ is the Banach space, or

$\|f\|_{q, u}:=\left[\sup _{x \in G} u(f(x))^{q} N_{\mu}(x)\right]^{1 / q}$ for each $u \in \mathcal{S}$, when $X$ is the complete $\mathbf{K}$-convex space. The completion of the space of all simple functions by $\|*\|_{q}$ or by $\left\{\|*\|_{q, u}: u \in \mathcal{S}\right\}$ denote by $L^{q}(\mu, X)$, where $L(\mu, X)=L^{1}(\mu, X)$.

Let $G$ be a totally disconnected completely regular space, let also $\mathrm{B}_{\mathrm{c}}(G)$ be a covering ring of clopen compact subsets in $G$, suppose that $\mu: \mathrm{B}_{\mathrm{c}}(G) \rightarrow \mathbf{K}$ is a finitely-additive function such that its restriction $\left.\mu\right|_{A}$ for each $A \in \mathrm{B}_{\mathrm{c}}(G)$ is a measure on a separating covering ring $\left.\mathcal{R}(G)\right|_{A}$, where $\left.\mathrm{B}_{\mathrm{c}}(G)\right|_{A}=\left.\mathcal{R}\right|_{A}$, $\left.\mathcal{R}\right|_{A}:=\{E \in \mathcal{R}: E \subseteq A\}$.

A measure $\eta: \mathcal{R} \rightarrow \mathbf{K}$ is called absolutely continuous relative to a measure $\mu: \mathcal{R} \rightarrow \mathbf{K}$, if there exists a function $f \in L(\mu, \mathbf{K})$ such that $\eta(A)=\int_{G} C h_{A}(x) f(x) \mu(d x)$ for each $A \in \mathcal{R}$, denote it by $\eta \preceq \mu$. If $\eta \preceq \mu$ and $\mu \preceq \eta$, then we say that $\eta$ and $\mu$ are equivalent $\eta \sim \mu$.

A $\mathbf{K}$-valued measure $P$ on $\mathcal{R}(X)$ we call a probability measure if $\|X\|_{P}=$ : $\|P\|=1$ and $P(X)=1$ (see $[32]$ ).

The following statements from the non-archimedean functional analysis proved in [37] are useful.

2. Lemma Let $\mu$ be a measure on $\mathcal{R}$. There exists a unique function $N_{\mu}: G \rightarrow[0, \infty)$ such that

(1) $\left\|C h_{A}\right\|_{N_{\mu}}=\|A\|_{\mu}$;

(2) if $\phi: G \rightarrow[0, \infty)$ and $\left\|C h_{A}\right\|_{\phi} \leq\|A\|_{\mu}$ for each $A \in \mathcal{R}$, then $\phi \leq N_{\mu}$; $N_{\mu}(x)=\inf _{x \in A, A \in \mathcal{R}}\|A\|_{\mu}$ for each $x \in X$.

3. Theorem. Let $\mu$ be a measure on $\mathcal{R}$. Then $\mathcal{R}_{\mu}$ is a covering ring of $G$ and $\bar{\mu}$ is a measure on $\mathcal{R}_{\mu}$ that extends $\mu$.

4. Lemma. If $\mu$ is a measure on $\mathcal{R}$, then $N_{\mu}=N_{\bar{\mu}}$ and $\mathcal{R}_{\mu}=\mathcal{R}_{\bar{\mu}}$.

5. Theorem. Let $\mu$ be a measure on $\mathcal{R}$, then $N_{\mu}$ is upper semicontinuous and for every $A \in \mathcal{R}_{\mu}$ and $\epsilon>0$ the set $\left\{x \in A: N_{\mu}(x) \geq \epsilon\right\}$ is $\mathcal{R}_{\mu}$-compact.

6. Theorem. Let $\mu$ be a measure on $\mathcal{R}$, let also $\mathcal{S}$ be a separating covering ring of $G$ which is a sub-ring of $\mathcal{R}_{\mu}$ and let $\nu$ be a restriction of $\mu$ 
onto $\mathcal{S}$. Then $\mathcal{S}_{\nu}=\mathcal{R}_{\mu}$ and $\bar{\nu}=\bar{\mu}$.

7. Notations and definitions. Let $(\Omega, \mathcal{A}, P)$ - be a probability space, where $\Omega$ is a space of elementary events, $\mathcal{A}$ is a separating covering ring of events in $\Omega, \mathcal{R}(\Omega) \subseteq \mathcal{A} \subseteq \mathcal{R}_{P}(\Omega), P: \mathcal{A} \rightarrow \mathbf{K}$ is a probability, $\mathbf{K}$ is a non-archimedean field of zero characteristic, $\operatorname{char}(\mathbf{K})=0$, complete relative to its multiplicative norm, $\mathbf{K} \supset \mathbf{Q}_{\mathbf{p}}, 1<p$ is a prime number, $\mathbf{Q}_{\mathbf{p}}$ is the field of $p$-adic numbers.

Denote by $\xi$ a random vector (a random variable for $n=1$ ) with values in $\mathbf{K}^{\mathbf{n}}$ or in a linear topological space $X$ over $\mathbf{K}$ such that it has the probability distribution $P_{\xi}(A)=P(\{\omega \in \Omega: \xi(\omega) \in A\})$ for each $A \in \mathcal{R}(X)$, where $\xi: \Omega \rightarrow \mathbf{X}, \xi$ is $(\mathcal{A}, \mathcal{R}(X))$-measurable, where $\mathcal{R}(X)$ is a separating covering ring of $X$ such that $\mathcal{R}(X) \subset \operatorname{Bco}(X), \operatorname{Bco}(X)$ denotes the separating covering ring of all clopen (simultaneously closed and open) subsets in $X$. That is, $\xi^{-1}(\mathcal{R}(X)) \subset \mathcal{A}$. If $T$ is a set and $\xi(t)$ is a random vector for each $t \in T$, then $\xi(t)$ is called a random function (or stochastic function). Particularly, if $T$ is a subset in a field, then $\xi(t)$ is called a stochastic process, while $t \in T$ is interpreted as the time parameter.

As usually put $M\left(\xi^{k}\right):=\int_{\Omega} \xi^{k}(\omega) P(d \omega)$ for a random variable $\xi$ and $k \in \mathbf{N}$ whenever it exists.

Random vectors $\xi$ and $\eta$ with values in $X$ are called independent, if $P(\{\xi \in A, \eta \in B\})=P(\{\xi \in A\}) P(\{\eta \in B\})$ for each $A, B \in \mathcal{R}(X)$.

8. Definition. Let $\{\Omega, \mathcal{R}, P\}$ be a probability space with a probability measure with values in a non-archimedean field $\mathbf{K}$ complete relative to its multiplicative norm, $\mathbf{K} \supset \mathbf{Q}_{\mathbf{p}}$. Consider a set $G$ and a ring $J$ of its subsets. Let $\xi(A)=\xi(\omega, A), \omega \in \Omega$, be a $\mathbf{K}$ - valued random variable for each $A \in J$ such that

$(M 1) \xi(A) \in Y, \xi(\emptyset)=0$, where $Y=L^{2}(\Omega, \mathcal{R}, P, \mathbf{K})$;

$(M 2) \xi\left(A_{1} \cup A_{2}\right)=\xi\left(A_{1}\right)+\xi\left(A_{2}\right) \bmod (P)$ for each $A_{1}, A_{2} \in J$ with $A_{1} \cap A_{2}=\emptyset$;

(M3) $M\left(\xi\left(A_{1}\right) \xi\left(A_{2}\right)\right)=\mu\left(A_{1} \cap A_{2}\right)$;

(M4) $M\left(\xi\left(A_{1}\right) \xi\left(A_{2}\right)\right)=0$ for each $A_{1} \cap A_{2}=\emptyset, A_{1}, A_{2} \in J$, that is $\xi\left(A_{1}\right)$ and $\xi\left(A_{2}\right)$ are orthogonal random variables, where $\mu(A) \in \mathbf{K}$ for each $A, A_{1}, A_{2} \in J$.

The family of random variables $\{\xi(A): A \in J\}$ satisfying Conditions $(M 1-M 4)$ we shall call the elementary orthogonal $\mathbf{K}$-valued stochastic measure.

9. Example. If $\xi(A)$ has a zero mean value $M \xi(A)=0$ for each 
$A \in J$, while $\xi\left(A_{1}\right)$ and $\xi\left(A_{2}\right)$ are independent random variables for $A_{1}, A_{2} \in$ $J$ with $A_{1} \cap A_{2}=\emptyset$, then they are orthogonal, since $M\left(\xi\left(A_{1}\right) \xi\left(A_{2}\right)\right)=$ $\left(M \xi\left(A_{1}\right)\right)\left(M \xi\left(A_{2}\right)\right)$.

10. Lemma. The function $\mu$ from Definition 8 is additive.

Proof. Since $\xi(A) \in Y=L^{2}(\Omega, \mathcal{R}, P, \mathbf{K})$ for each $A \in J$, then there exists $M \xi(A)=\int_{\Omega} \xi(\omega, A) P(d \omega)$, since $\sup _{x \in G}|\xi(\omega, A)|^{2} N_{P}^{2}(\omega) \leq \sup _{x \in G}|\xi(\omega, A)|^{2} N_{P}(\omega)$ for the probability measure $P$ having $N_{p}(x) \leq 1$ for each $x \in G$, that is, $L^{1}(\Omega, \mathcal{R}, P, \mathbf{K}) \subset L^{2}(\Omega, \mathcal{R}, P, \mathbf{K})$.

Therefore, from Conditions $(M 2, M 4)$ for each $A_{1}, A_{2} \in J$ with the void intersection $A_{1} \cap A_{2}=\emptyset$ the equalities follow:

$$
M\left(\xi^{2}\left(A_{1} \cup A_{2}\right)\right)=M\left[\left(\xi\left(A_{1}\right)+\xi\left(A_{2}\right)\right)^{2}\right]
$$

$=M\left[\xi^{2}\left(A_{1}\right)+2 \xi\left(A_{1}\right) \xi\left(A_{2}\right)+\xi^{2}\left(A_{2}\right)\right]=M \xi^{2}\left(A_{1}\right)+M \xi^{2}\left(A_{2}\right)$. In view of $(M 3)$ this gives

$\mu\left(A_{1} \cup A_{2}\right)=\mu\left(A_{1}\right)+\mu\left(A_{2}\right)$.

11. Note. Suppose that $\mu$ has an extension to a measure on the separating covering ring $\mathcal{R}(G), G$ is a totally disconnected completely regular space, where $J \subset \mathcal{R}_{\mu}(G)$.

12. Definitions. Let a random function $\xi(t)$ be with values in a complete linear locally $\mathbf{K}$-convex space $X$ over $\mathbf{K}, t \in T$, where $(T, \rho)$ is a metric space with a metric $\rho$. Then $\xi(t)$ is called stochastically continuous at a point $t_{0}$, if for each $\epsilon>0$ there exists $\lim _{\rho\left(t, t_{0}\right) \rightarrow 0} P\left(\left\{u\left(\xi(t)-\xi\left(t_{0}\right)\right)>\epsilon\right\}\right)=0$ for each $u \in \mathcal{S}$. If $\xi(t)$ is stochastically continuous at each point of a subset $E$ in $T$, then it is called stochastically continuous on $E$.

If $\lim _{R \rightarrow \infty} \sup _{t \in E} P(\{u(\xi(t))>R\})=0$ for each $u \in \mathcal{S}$, then a random function $\xi(t)$ is called stochastically bounded on $E$.

Let $L^{0}(\mathcal{R}(G), X)$ denotes the class of all step (simple) functions $f(x)=$ $\sum_{k=1}^{m} c_{k} C h_{A_{k}}(x)$, where $c_{k} \in X, A_{k} \in \mathcal{R}(G)$ for each $k=1, \ldots, m \in \mathbf{N}$, $A_{k} \cap A_{j}=\emptyset$ for each $k \neq j$. Then the non-archimedean stochastic integral by the elementary orthogonal stochastic measure $\xi(A)$ of $f \in L^{0}(\mathcal{R}(G), X)$ is defined by the formula:

$(S I) \eta(\omega):=\int_{G} f(x) \xi(\omega, d x):=\sum_{k=1}^{m} c_{k} \xi\left(\omega, A_{k}\right)$.

13. Lemma. Let $f, g \in L^{0}(\mathcal{R}(G), \mathbf{K})$, where $f(x)=\sum_{k=1}^{m} c_{k} C h_{A_{k}}(x)$ and $g(x)=\sum_{k=1}^{m} d_{k} C h_{A_{k}}(x)$, then $M\left(\int_{G} f(x) \xi(d x) \int_{G} g(y) \xi(d y)\right)=\sum_{k=1}^{m} c_{k} d_{k} \mu\left(A_{k}\right)$ and there exists a $\mathbf{K}$-linear embedding of $L^{0}(\mathcal{R}(G), \mathbf{K})$ into $L^{2}(\mu, \mathbf{K})$.

Proof. In view of Conditions $(M 1, M 2)$ there exists $\int_{G} f(x) \xi(\omega, d x) \in$ $Y=L(P)$. Since $\left.\int_{G} f(x) \xi(d x) \int_{G} g(y) \xi(d y)=\sum_{k, j=1}^{m} c_{k} d_{j} \xi A_{k}\right) \xi\left(A_{j}\right.$, then 
$M\left(\int_{G} f(x) \xi(d x) \int_{G} g(y) \xi(d y)\right)=\sum_{k, j=1}^{n} c_{k} d_{j} M\left(\xi\left(A_{k}\right) \xi\left(A_{j}\right)\right)=\sum_{k=1}^{m} c_{k} d_{k} \mu\left(A_{k}\right)$ due to Conditions $(M 3, M 4)$, since $A_{k} \cap A_{j}=\emptyset$ for each $j \neq k$. This gives the $\mathbf{K}$-linear embedding $\theta$ of $L^{0}(\mathcal{R}(G), \mathbf{K})$ into $L^{2}(\mu, \mathbf{K})$ such that $\theta(f)=\sum_{k=1}^{n} c_{k} C h_{A_{k}}(x)$ and

(i) $\|\theta(f)\|_{2}=\left[\max _{k=1}^{m}\left|c_{k}\right|^{2} \sup _{x \in A_{k}} N_{\mu}(x)\right]^{1 / 2}=\left[\max _{k=1}^{m}\left|c_{k}\right|^{2}\left\|A_{k}\right\|_{\mu}\right]^{1 / 2}<$ $\infty$ due to Lemma 2.

14. Note. Denote by $L^{2}(\mathcal{R}(G), \mathbf{K})$ the completion of $L^{0}(\mathcal{R}(G), \mathbf{K})$ by the norm $\|*\|_{2}$ induced from $L^{2}(\mu, \mathbf{K})$.

15. Definition. Let $L^{0}(\xi, X)$ denotes the class of all step (simple) functions $f(x)=\sum_{k=1}^{m} c_{k} \xi\left(A_{k}\right)$, where $c_{k} \in X, A_{k} \in \mathcal{R}(G)$ for each $k=$ $1, \ldots, m \in \mathbf{N}, A_{k} \cap A_{j}=\emptyset$ for each $k \neq j$. Then the non-archimedean stochastic integral by the elementary orthogonal stochastic measure $\xi(A)$ of $f \in L^{0}(\xi, X)$ is defined by the formula:

(SI) $\eta(\omega):=\int_{G} f(x) \xi(\omega, d x):=\sum_{k=1}^{m} c_{k} \xi\left(\omega, A_{k}\right)$.

16. Lemma. Let $f, g \in L^{0}(\xi, \mathbf{K})$, where $f(x)=\sum_{k=1}^{m} c_{k} \xi\left(A_{k}\right)$ and $g(x)=\sum_{k=1}^{m} d_{k} \xi\left(A_{k}\right)$, then $M\left(\int_{G} f(x) \xi(d x) \int_{G} g(y) \xi(d y)\right)=\sum_{k=1}^{m} c_{k} d_{k} \mu\left(A_{k}\right)$ and there exists a $\mathbf{K}$-linear embedding of $L^{0}(\mathcal{R}(G), \mathbf{K})$ into $L^{2}(P, \mathbf{K})$.

Proof. In view of Conditions $(M 1, M 2)$ there exists $\int_{G} f(x) \xi(\omega, d x) \in$ $Y=L(P, \mathbf{K})$. But $f$ is the step function, hence

(i) $\|f\|_{L^{2}(P, \mathbf{K})}=\left[\max _{k=1}^{m}\left|c_{k}\right|^{2} \sup _{\omega \in \Omega}\left|\xi^{2}\left(\omega, A_{k}\right)\right| N_{P}(x)\right]^{1 / 2}$ $=\left[\max _{k=1}^{m}\left|c_{k}\right|^{2}\left\|\xi\left(*, A_{k}\right)\right\|_{L^{2}(P)}^{2}\right]^{1 / 2}<\infty$ and inevitably $f \in L^{2}(P)$. Thus the mapping $\psi(f):=\sum_{k=1}^{m} c_{k} C h_{A_{k}}(x)$ gives the $\mathbf{K}$-linear embedding of $L^{0}(\xi, \mathbf{K})$ into $L^{2}(P, \mathbf{K})$. The second statement is verified as in Lemma 13 due to Formulas 12, 15(SI).

17. Note. Denote by $L^{2}(\xi, \mathbf{K})$ the completion of $L^{0}(\xi, \mathbf{K})$ by the norm $\|*\|_{2}$ induced from $L^{2}(P, \mathbf{K})$.

18. Corollary. The mappings $12(S I)$ and $15(S I)$ and Conditions (M1M4) induce an isometry between $L^{2}(\mathcal{R}(G), \mathbf{K})$ and $L^{2}(\xi)$.

Proof. The valuation group $\Gamma_{\mathbf{K}}:=\{|z|: z \in \mathbf{K}, z \neq 0\}$ is contained in $(0, \infty)$. In view of Theorem 5 , Lemma 10 and Note 11 without loss of generality for a step function $f$ we take a representation with $A_{k} \in \mathcal{R}(G)$ such that $\left\|A_{k}\right\|_{\mu}=\left|\mu\left(A_{k}\right)\right|$ for each $k=1, \ldots, m$. The family of all such step functions is everywhere dense in $L^{2}(\mathcal{R}(G), \mathbf{K})$.

Since $M\left(\xi^{2}(A)\right)=\mu(A)$ for each $A \in \mathcal{R}(G)$, then $N_{\mu}(x)=\inf _{A \in \mathcal{R}(G), x \in A}\|A\|_{\mu}$, where $\|A\|_{\mu}=\sup \{|\mu(B)|: B \in \mathcal{R}(G), B \subset A\}=\sup \left\{\left|M\left(\xi^{2}(B)\right)\right|\right.$ : $B \in \mathcal{R}(G), B \subset A\}$. On the other hand, $M\left(\xi^{2}(B)\right)=\int_{\Omega} \xi^{2}(\omega, B) P(d \omega)$, 
$\left|M\left(\xi^{2}(B)\right)\right| \leq \sup _{\omega \in \Omega}\left|\xi^{2}(\omega, B)\right| N_{P}(\omega)$. By our supposition $\mu$ is the measure, hence taking a shrinking family $\mathcal{S}$ in $\mathcal{R}(G)$ such that $\bigcap_{A \in \mathcal{S}} A=\{x\}$ we get

$$
N_{\mu}(x)=\inf _{A \in \mathcal{R}(G), x \in A}\left[\sup _{B \in \mathcal{R}(G), B \subset A} \sup _{\omega \in \Omega}|\xi(\omega, B)|^{2} N_{P}(\omega)\right] .
$$

Thus $N_{\mu}(x)=\inf _{A \in \mathcal{R}(G), x \in A}\left[\sup _{B \in \mathcal{R}(G), B \subset A}\left\|\xi^{2}(*, B)\right\|_{L^{2}(P)}\right]$ and $\left\|A_{k}\right\|_{\mu}=$ $\left\|\xi\left(*, A_{k}\right)\right\|_{L^{2}(P)}^{2}$ for each $k=1, \ldots, m$ due to Lemma 2 and due to the choice $\left\|A_{k}\right\|_{\mu}=\left|\mu\left(A_{k}\right)\right|$ above.

The mapping $\psi$ from $\S 16$ also is $\mathbf{K}$-linear from $L^{0}(\xi)$ into $L^{0}(\mathcal{R}(G), \mathbf{K})$ such that $\psi$ is the isometry relative to $\|*\|_{L^{2}(P)}$ and $\|*\|_{L^{2}(\mu)}$ due to Formulas $13(i)$ and $16(i)$ and Lemma 2. Two spaces $L^{2}(P)$ and $L^{2}(\mu)$ are complete by their definitions, consequently, $\psi$ has the $\mathbf{K}$-linear extension from $L^{2}(\mathcal{R}(G), \mathbf{K})$ onto $L^{2}(\xi)$ which is the isometry between $L^{2}(\mathcal{R}(G), \mathbf{K})$ and $L^{2}(\xi)$.

19. Definition. If $f \in L^{2}(\mathcal{R}(G), \mathbf{K})$, then put by the definition:

$\eta=\psi(f)=\int_{G} f(x) \xi(d x)$.

The random variable $\eta$ we call the non-archimedean stochastic integral of the function $f$ by measure $\xi$.

Taking a limit in $L^{2}(P, X)$ we denote also by l.i.m.

20. Theorems. 1. For a step function $f(x)=\sum_{k=1}^{n} a_{k} C h_{A_{k}}(x)$, where $a_{k} \in \mathbf{K}, A_{k} \in \mathcal{R}(G), n=n(f) \in \mathbf{N}$, the stochastic integral is given by the formula:

$\eta=\int f(x) \xi(d x)=\sum_{k=1}^{n} a_{k} \xi\left(A_{k}\right)$.

2. For each $f, g \in L^{2}(\mathcal{R}(G), \mathbf{K})$ there is the identity:

$M\left(\int_{G} f(x) \xi(d x) \int_{G} g(y) \xi(d y)\right)=\int_{G} f(x) g(x) \mu(d x)$.

3. For each $f, g \in L^{2}(\mathcal{R}(G), \mathbf{K})$ and $\alpha, \beta \in \mathbf{K}$ the stochastic integral is $\mathbf{K}$-linear:

$\int_{G}[\alpha f(x)+\beta g(x)] \xi(d x)=\alpha \int_{G} f(x) \xi(d x)+\beta \int_{G} g(x) \xi(d x)$.

4. For each sequence of functions $f_{n} \in L^{2}(G, \mathcal{R}(G), \mu, \mathbf{K})$ such that $\lim _{n \rightarrow \infty}\left\|f-f_{n}\right\|_{L^{2}(\mu, \mathbf{K})}=0$ there is exists the limit:

$\int_{G} f(x) \xi(d x)=$ l.i.m.n . $_{\text {. }} \int_{G} f_{n}(x) \xi(d x)$.

5. There exists an extension of $\xi$ from $\mathcal{R}$ onto $\mathcal{R}_{\mu}(G)$.

Proof. Statements of (1) and (3) follow from the consideration above. To finish the proof of (2) it is sufficient to show that $f g \in L^{1}(\mu, \mathbf{K})$, if $f$ and $g \in L^{2}(\mu, \mathbf{K})$, where $\mu$ is the measure on $G$. Since $2|f(x) g(x)| \leq|f(x)|^{2}+$ $|g(x)|^{2}$ for each $x \in G$, then $2 \sup _{x \in G}|f(x) g(x)| N_{\mu}(x) \leq \sup _{x \in G}\left(|f(x)|^{2}+\right.$ $\left.|g(x)|^{2}\right) N_{\mu}(x) \leq\|f\|_{L^{2}(\mu)}^{2}+\|g\|_{L^{2}(\mu)}^{2}$, consequently, $f(x) g(x)$ is $\mu$-integrable.

4. From $\lim _{n \rightarrow \infty}\left[\sup _{x \in G}\left|f(x)-f_{n}(x)\right|^{2} N_{\mu}(x)\right]=0$ and $M\left[\int_{G}\left(f-f_{n}\right)(x) \xi(d x) \int_{G}(f-\right.$ 
$\left.\left.f_{n}\right)(y) \xi(d y)\right]=\int_{G}\left(f-f_{n}\right)^{2}(x) \mu(d x)$ it follows, that $\lim _{n \rightarrow \infty} M\left[\left(\int_{G}\left(f-f_{n}\right)(x) \xi(d x)\right)^{2}\right]=$ 0, that is l.i.m.n $\cdot_{n \rightarrow \infty} \int_{G} f_{n}(x) \xi(d x)=\int_{G} f(x) \xi(d x)$ due to Corollary 18.

5. Extend now the stochastic measure $\xi$ from $\mathcal{R}(G)$ to $\tilde{\xi}$ on $\mathcal{R}_{\mu}(G)$. If $A \in \mathcal{R}_{\mu}(G)$, then $C h_{A} \in L(G, \mathcal{R}(G), \mu, \mathbf{K})$. Since $C h_{A} \in L(G, \mathcal{R}(G), \mu, \mathbf{K})$, then $\sup _{x \in A} N_{\mu}(x)<\infty$. Put $\tilde{\xi}(A):=\int_{G} C h_{A}(x) \xi(d x)=\int_{A} \xi(d x)$ for each $A \in \mathcal{R}_{\mu}(G)$, consequently,

(1) $\tilde{\xi}$ is defined on $\mathcal{R}_{\mu}(G)$.

Therefore, $\tilde{\xi}(A)=\xi(A)$ for each $A \in \mathcal{R}(G)$. For each $A, B \in \mathcal{R}_{\mu}(G)$ there exist sequences of simple functions $f_{n}=\sum_{k} a_{k, n} C h_{A_{k, n}}, g_{m}=\sum_{l} b_{l, m} C h_{B_{l, m}}$ with $a_{k, n}, b_{l, m} \in \mathbf{K}, A_{k, n}, B_{l, m} \in \mathcal{R}(G)$ such that $\lim _{n \rightarrow \infty}\left\|C h_{A}-f_{n}\right\|_{L(\mu)}=$ 0 and $\lim _{m \rightarrow \infty}\left\|C h_{B}-g_{m}\right\|_{L(\mu)}=0$. Since $M\left(a_{k, n} \tilde{\xi}\left(A_{k, n}\right) b_{l, m} \tilde{\xi}\left(B_{l, m}\right)\right)=$ $a_{k, n} b_{l, m} \mu\left(A_{k, n} \cap B_{l, m}\right)$ for each $k, n, l, m$, then

(2) $M(\tilde{\xi}(A) \tilde{\xi}(B))=\bar{\mu}(A \cap B)$ for each $A, B \in \mathcal{R}_{\mu}(G)$, where $\bar{\mu}$ is the extension of the measure $\mu$ from $\mathcal{R}(G)$ on $\mathcal{R}_{\mu}(G)$. If $\mathcal{S} \subset \mathcal{R}_{\mu}(G)$ is a shrinking family such that $\bigcap_{A \in \mathcal{S}} A=\emptyset$, then

(3) l.i.m. A $_{\mathcal{S}} \tilde{\xi}(A)=0$ due to Corollary 18, since $M[\tilde{\xi}(A)]^{2}=\bar{\mu}(A)$ and $\lim _{A \in \mathcal{S}} \bar{\mu}(A)=0$ due to Theorem 3 .

21. Definition. A random function of sets satisfying conditions $20.5(1-$ 3 ) is called the orthogonal stochastic measure.

22. Corollary. Let $\xi$ and $\tilde{\xi}$ be as in Theorem 20.5, then $L^{2}(\xi, \mathbf{K})=$ $L^{2}(\tilde{\xi}, \mathbf{K})$.

23. Note. If $\xi$ is an orthogonal stochastic measure with a structure measure $\mu$ on $\mathcal{R}_{\mu}(G)$ and $g \in L^{2}(\mu, \mathbf{K})$, then put $\rho(A):=\int_{G} C h_{A}(x) g(x) \xi(d x)$ for each $A \in \mathcal{R}_{\mu}(G)$ and $\nu(A):=\int_{A} g^{2}(x) \mu(d x)$.

24. Lemma. If $f \in L^{2}(\nu, \mathbf{K})$, then $f(x) g(x) \in L^{2}(\mu, \mathbf{K})$ and $\int_{G} f(x) \rho(d x)=$ $\int_{G} f(x) g(x) \xi(d x)$.

Proof. In view of Theorems 20 for each $A, B \in \mathcal{R}_{\mu}(G)$ there is the equality

$$
M[\rho(A) \rho(B)]=M\left[\int_{G} C h_{A}(x) g(x) \xi(d x) \int_{G} C h_{B}(y) g(y) \xi(d y)\right.
$$

$=\int_{A \cap B} g^{2}(x) \mu(d x)=\nu(A \cap B)$.

Since $g \in L^{2}(\mu, \mathbf{K})$, then $\nu$ is the measure absolutely continuous relative to $\mu$ on $\mathcal{R}_{\mu}(G)$. If $f(x)=\sum_{k} a_{k} C h_{A_{k}}(x)$ is a simple function with $a_{k} \in$ $\mathbf{K}$ and $A_{k} \in \mathcal{R}_{\mu}(G)$, then $\int_{G} f(x) \rho(d x)=\sum_{k} a_{k} \int_{G} C h_{A_{k}}(x) g(x) \xi(d x)=$ $\sum_{k} a_{k} \rho\left(A_{k}\right)=\int_{G} f(x) g(x) \xi(d x)$, since

$\sup _{x \in G}|f(x) g(x)|^{2} N_{\mu}(x) \leq\left[\max _{k}|a|_{k}^{2}\right] \sup _{x \in G}|g(x)|^{2} N_{\mu}(x)<\infty$.

If $f_{n}$ is a fundamental sequence of simple functions in $L^{2}(\nu, \mathbf{K})$, then $M\left[\left(\int_{G}\left(f_{n}-\right.\right.\right.$ 
$\left.\left.\left.f_{m}\right)(x) \rho(d x)\right)^{2}\right]=\int_{G}\left[\left(f_{n}-f_{m}\right)(x)\right]^{2} g^{2}(x) \mu(d x)$, hence $f_{n} g$ is the fundamental sequence in $L^{2}(\mu, \mathbf{K})$. Therefore, there exists

$$
\lim _{n \rightarrow \infty} \int_{G} f_{n}(x) \rho(d x)=\lim _{n \rightarrow \infty} \int_{G} f_{n}(x) g(x) \xi(d x),
$$
consequently, $\int_{G} f(x) \rho(d x)=\int_{G} f(x) g(x) \xi(d x)$.

25. Lemma. If $A \in \mathcal{R}_{\mu}(G)$, then $\xi(A)=\int_{G}\left[C h_{A}(x) / g(x)\right] \rho(d x)$.

Proof. Since $\nu(\{x: g(x)=0\})=0$, then $1 / g(x)$ is defined $\nu$-almost everywhere on $G$, hence $\int_{G}\left[C h_{A}(x) / g^{2}(x)\right] \nu(d x)=\int_{G}\left[g^{2}(x) / g^{2}(x)\right] \mu(d x)=$ $\mu(A)$. In view of Lemma $24 \int_{G}\left[C h_{A}(x) / g(x)\right] \rho(d x)=\int_{G}\left[C h_{A}(x) g(x) / g(x)\right] \xi(d x)=$ $\xi(A)$.

26. Notation and Remark. Let $T$ be a totally disconnected Hausdorff topological space with a separating covering $\operatorname{ring} \mathcal{R}(T)$ and with a non-trivial measure $h: \mathcal{R}(T) \rightarrow \mathbf{K}$. Denote by $B(X, x, R):=\{y \in X: \rho(x, y) \leq R\}$ the ball in a metric space $(X, \rho)$ with a metric $\rho, 0<R<\infty$. In particular, $T$ may be either a clopen subset in $\mathbf{K}_{\mathbf{r}}$ or a segment in $\mathbf{R}, h$ may be a nontrivial $\mathbf{K}$-valued measure on a separating covering ring $\mathcal{R}\left(B\left(T, t_{0}, R\right)\right)$ for each $t_{0} \in T$ and every $0<R<\infty$, where $\mathcal{R}\left(B\left(T, t_{0}, R_{1}\right)\right) \subset \mathcal{R}\left(B\left(T, t_{0}, R_{2}\right)\right)$ for each $0<R_{1}<R_{2}<\infty$ and each $t_{0} \in T, \mathbf{K} \supset \mathbf{Q}_{\mathbf{p}}, \mathbf{K}_{\mathbf{r}} \supset \mathbf{Q}_{\mathbf{p}^{\prime}}, r=p^{\prime}, r$ and $p$ are primes, $\mathbf{K}$ and $\mathbf{K}_{\mathbf{r}}$ are non-archimedean fields complete relative to their multiplicative norms.

There exists a continuous mapping from a clopen subset in $\mathbf{Q}_{\mathbf{p}}^{\prime}$ onto $[a, b]$ in $\mathbf{R},-\infty<a<b<\infty$ (see [10]), hence a suitable separating covering ring $\mathcal{R}(T)$ and $h$ exist on $[a, b]$.

Recall that a measure $h: \mathbf{B}_{\mathrm{c}}\left(\mathbf{K}_{\mathbf{r}}\right) \rightarrow \mathbf{K}$ is called the Haar measure, if $h(t+B)=h(B)$ for each clopen compact subset $B$ in $\mathbf{K}_{\mathbf{r}}$ and each $t \in \mathbf{K}_{\mathbf{r}}$. The Haar measure exists due to the Monna-Springer Theorem 8.4 [37], when $r \neq p$ are mutually prime, $(r, p)=1$, since $B\left(\mathbf{K}_{\mathbf{r}}, 0, R\right)$ is $p$-free. For example, there can be taken a non-trivial $\mathbf{K}$-valued Haar measure $h$ on $\mathbf{B}_{\mathrm{c}}\left(\mathbf{K}_{\mathbf{r}}\right)$ such that $h\left(B\left(\mathbf{K}_{\mathbf{r}}, 0,1\right)\right)=1$, but generally we do not demand, that $h$ is a Haar measure or $r \neq p$.

Suppose that a function $g(t, x)$ on $T \times G$ is $\mathcal{R}_{h} \times \mathcal{R}_{\mu}$-measurable and $g \in L^{2}\left(T \times G, \mathcal{R}_{h} \times \mathcal{R}_{\mu}, h \times \mu, \mathbf{K}\right)$, where $\mathcal{R}_{h}:=\mathrm{B}_{c h}\left(\mathbf{K}_{\mathbf{r}}\right)$.

27. Lemma. The stochastic integral

(1) $\rho(t)=\int_{G} g(t, x) \xi(d x)$ is defined for each $t \in T$ for P-almost all $\omega \in \Omega$ and it can be defined such that the stochastic function $\rho(t)$ would be measurable.

Proof. If $g(t, x)=\sum_{k} a_{k} C h_{B_{k}}(t) C h_{A_{k}}(x)$ is a simple function with $A_{k} \in \mathcal{R}_{\mu}$ and $B_{k} \in \mathcal{R}_{h}$ and $a_{k} \in \mathbf{K}$ for each $k=1, \ldots, m, m \in \mathbf{N}$, 
then $\rho(t)=\sum_{k} a_{k} C h_{B_{k}}(t) \xi\left(A_{k}\right)$ is $\mathcal{R}_{h} \times \mathcal{A}$-measurable function of variables $(t, \omega) \in T \times \Omega$ (see also Definitions 7$)$. For each $g \in L^{2}(h \times \mu, \mathbf{K})$ there exists a sequence of simple functions $g_{n}(t, x)$ such that $\lim _{n \rightarrow \infty} \sup _{t \in T, x \in G} \mid g(t, x)-$ $\left.g_{n}(t, x)\right|^{2} N_{h}(t) N_{\mu}(x)=0$.

Let $\rho_{n}(t):=\int_{G} g_{n}(t, x) \xi(d x)$, then there exists a stochastic function $\tilde{\rho}(t)$ such that $\lim _{n \rightarrow \infty} \sup _{t \in T}\left|M\left[\left(\tilde{\rho}(t)-\rho_{n}(t)\right)^{2}\right]\right| N_{h}(t)=0$. There is the equality $\int_{T} M\left[\left(\tilde{\rho}(t)-\rho_{n}(t)\right)^{2}\right] h(d t)=\int_{T} \int_{G}\left[g(t, x)-g_{n}(t, x)\right]^{2} \mu(d x) h(d t)$, hence $\sup _{t \in T}\left|M\left[\left(\tilde{\rho}(t)-\rho_{n}(t)\right)^{2}\right]\right| N_{h}(t) \leq \sup _{t \in T, x \in G}\left|g(t, x)-g_{n}(t, x)\right|^{2} N_{h}(t) N_{\mu}(x)<$ $\infty$ and inevitably the stochastic function $\tilde{\rho}(t)$ is $\mathcal{R}_{h} \times \mathcal{A}$-measurable by $(t, \omega) \in T \times \Omega$ and it exists with the unit probability. Thus $h(\{t \in A$ : $\left.\left.M\left[(\rho(t)-\tilde{\rho}(t))^{2}\right]=0\right\}\right)=h(A)$ for each $A \in \mathcal{R}(T)$.

Finally put $\eta(t)=\tilde{\rho}(t)$ if $P(\{\rho(t) \neq \tilde{\rho}(t)\})=0$, while $\eta(t)=\rho(t)$ if $P(\{\rho(t) \neq \tilde{\rho}(t)\}) \neq 0$. Therefore, the stochastic function $\eta$ is $\mathcal{R}_{h} \times \mathcal{A}$ measurable, since $\eta$ differs from $\mathcal{R}_{h} \times \mathcal{A}$-measurable function $\tilde{\rho}(t)$ on a set of zero $h \times P$-measure and $\eta$ is stochastically equivalent with $\rho$.

28. Remark. Henceforth, due to Lemma 27 we shall suppose that the stochastic integrals $27(1)$ are $\mathcal{R}_{h} \times \mathcal{A}$-measurable.

29. Lemma. If $g(t, y)$ and $z(t)$ are $\mathcal{R}_{h} \times \mathcal{R}_{\mu}$ and $\mathcal{R}_{h}$-measurable functions, $g \in L^{2}\left(T \times \mathbf{K}, \mathcal{R}_{h} \times \mathcal{R}_{\mu}, h \times \mu, \mathbf{K}\right)$ and $z \in L^{2}\left(T, \mathcal{R}_{h}, h, \mathbf{K}\right)$, $\xi$ is an orthogonal stochastic measure on $\left(\mathbf{K}, \mathcal{R}_{\mu}\right)$, then

(1) $\int_{T} z(t) \int_{\mathbf{K}} g(t, y) \xi(d y) h(d t)=\int_{\mathbf{K}} q(y) \xi(d y)$, where $q(y)=\int_{T} z(t) g(t, y) h(d t)$.

Proof. Since $z \in L^{2}(h)$ and $g \in L^{2}(h \times \mu)$, then

(2) $\sup _{t \in T, y \in \mathbf{K}}|z(t) g(t, y)|^{2} N_{h}^{2}(t) N_{\mu}(y) \leq$

$\left[\sup _{t \in T}|z(t)|^{2} N_{h}(t)\right] \sup _{t \in T, y \in \mathbf{K}}|g(t, y)|^{2} N_{h}(t) N_{\mu}(y)<\infty$.

Consider $g \in L^{2}(h \times \mu)$ and a sequence $g_{n}(t, y)=\sum_{k} a_{k, n} C h_{B_{k, n}}(t) C h_{A_{k, n}}(y)$ of step functions converging to $g$ in $L^{2}(h \times \mu, \mathbf{K})$, where $a_{k, n} \in \mathbf{K}, A_{k, n} \in \mathcal{R}_{\mu}$, $B_{k, n} \in \mathcal{R}_{h}$ for each $k, n$. The mean value of the square of the left side of Equation (1) is:

(3) $M\left(\left[\int_{T} z(t) \int_{\mathbf{K}} g(t, y) \xi(d y) h(d t)\right]^{2}\right)$

$=\left(\int_{T} \int_{T} z\left(t_{1}\right) z\left(t_{2}\right) \int_{\mathbf{K}} g\left(t_{1}, y\right) g\left(t_{2}, y\right) \mu(d y) h\left(d t_{1}\right) h\left(d t_{2}\right)\right.$

$=\int_{\mathbf{K}}\left[\int_{T} z(t) g(t, y) h(d t)\right]^{2} \mu(d y)$.

Equation (1) is satisfied for step functions. In view of (3) the left an the right sides of (1) are continuous relative to taking a limit by $g_{n}(t, y)$ in $L^{2}(h \times \mu, \mathbf{K})$ in the mean square sense relative to the probability $P$ as well as in the space $L^{2}(P, \mathbf{K})$. Since the family of step functions is dense in $L^{2}(h \times \mu, \mathbf{K})$, then the statement of this Lemma follows. 
30. Remark and Notation. If conditions of Lemma 29 are satisfied for each $T=B\left(\mathbf{K}_{\mathbf{r}}, 0, R\right)$, or $T=[-R, R]$ respectively, $0<R<\infty$ and if there exists

$$
\int_{\mathbf{K}_{\mathbf{r}}} z(t) g(t, y) h(d t)=\lim _{R \rightarrow \infty} \int_{B\left(\mathbf{K}_{\mathbf{r}}, 0, R\right)} z(t) g(t, y) h(d t)
$$

in $L^{2}(\mu)$, then

(1) $\int_{\mathbf{K}_{\mathbf{r}}} z(t) \int_{\mathbf{K}} g(t, y) \xi(d y) h(d t)=\int_{\mathbf{K}} s(y) \xi(d y)$,

where $s(y):=\int_{\mathbf{K}_{\mathbf{r}}} z(t) g(t, y) h(d t)$. This follows from Lemma 29, since the left side of 30(1) is the limit of the left side of 29(1), when $R$ tends to the infinity. In the right side of $29(1)$ it is possible to take the limit under the sign of the stochastic integral in the mean square sense relative to the probability $P$ as well as in the space $L^{2}(P)$.

Describe now the generalization of the above construction onto K-linear spaces $X$, which may be infinite-dimensional over $\mathbf{K}$.

\section{$3 \quad$ Vector spectral functions}

31. Let $X$ be a complete locally $\mathbf{K}$-convex space over an infinite field $\mathbf{K}$ of zero characteristic, $\operatorname{char}(\mathbf{K})=0$, with a non-archimedean multiplicative norm relative to which $\mathbf{K}$ is complete. Then the space $\operatorname{Lin}(X, X)=\operatorname{Lin}(X)$ of all $\mathbf{K}$-linear continuous operators $F: X \rightarrow X$ is locally $\mathbf{K}$ convex and complete. A K-linear continuous operator $F$ is called compact, if for each $\epsilon>0$ there exists a finite-dimensional over $\mathbf{K}$ vector subspace $X_{\epsilon}$ such that it has a complement $Z_{\epsilon}:=X \ominus X_{\epsilon}$ in $X$ and $u(F x) \leq \epsilon u(x)$ for each $x \in Z_{\epsilon}$ and each semi-norm $u$ in $X$, where $Z_{\epsilon}$ is the $\mathbf{K}$-vector subspace in $X$ such that $Z_{\epsilon} \cap X_{\epsilon}=\{0\}, Z_{\epsilon} \oplus X_{\epsilon}=X$. Consider the subspace $\operatorname{Lc}(X)$ in $\operatorname{Lin}(X)$ of all compact operators in $X$.

Let $W_{1}: X \rightarrow X^{T}$ and $W_{2}: \operatorname{Lin}(X) \rightarrow \operatorname{Lin}(X)$ be linear isomorphisms of transposition denoted simply by $W$ such that a restriction of $W$ on each finite-dimensional subspace $\mathbf{K}^{\mathbf{n}}$ in $X$ or $\operatorname{Mat}_{n}(\mathbf{K})$ in $\operatorname{Lin}(\mathbf{K})$ gives $W(F)$ a transposed vector or matrix, where $\operatorname{Mat}_{n}(\mathbf{K})$ denotes the $\mathbf{K}$-linear space of all $n \times n$ matrices with entries in $\mathbf{K}$. Suppose that there exist $\mathbf{K}$-linear continuous multiplications

(T1) $X \times X \ni\left\{a, b^{T}\right\} \mapsto(a, b) \in \mathbf{K}$ and

(T2) $X \times X \ni\left\{a^{T}, b\right\} \mapsto[a, b] \in L c(X)$, where $b^{T}:=W(b)$.

31.1. Examples. Let $X=c_{0}(\alpha, \mathbf{K})$ be the Banach space consisting 
of vectors $x=\left(x_{j}: j \in \alpha, x_{j} \in \mathbf{K}\right)$ such that for each $\epsilon>0$ the set $\left\{j:\left|x_{j}\right|>\epsilon\right\}$ is finite with the norm $\|x\|_{c_{0}}:=\sup _{j \in \alpha}\left|x_{j}\right|$, where $\alpha$ is a set. Due to the Zermelo Theorem (see [10]) as $\alpha$ it can be taken an ordinal. This Banach space $c_{0}(\alpha, \mathbf{K})$ has the standard basis $\left\{e_{j}: j \in \alpha\right\}$, where $e_{j}=(0, \ldots, 0,1,0, \ldots)$ with 1 in the $j$-th place and others entries zero. This basis is orthonormal in the non-archimedean sense [37].

Then for each $F \in \operatorname{Lin}(X)$ there are $F_{i, j} \in \mathbf{K}$ such that

(1) $F e_{i}=\sum_{j \in \alpha} F_{i, j} e_{j}$ for each $i \in \alpha$.

If $F \in L c(X)$, then for each $\epsilon>0$ the set $\beta(F):=\left\{(i, j):\left|F_{i, j}\right|>\epsilon, i, j \in \alpha\right\}$ is finite, where $X_{\epsilon}=\operatorname{span}_{\mathbf{K}}\left\{e_{j}: \exists(i, j) \vee(j, i) \in \beta(F)\right\}, \operatorname{span}_{\mathbf{K}}\left\{y_{j}: j \in\right.$ $\beta\}:=\left\{z=a_{1} y_{j_{1}}+\ldots+a_{k} y_{j_{k}}: a_{1}, \ldots, a_{k} \in \mathbf{K}, k \in \mathbf{N}, j_{1}, \ldots, j_{k} \in \beta\right\}$ denotes the $\mathbf{K}$-linear span of vectors.

If $x$ is a row-vector, then $W(x)$ is a column-vector. If $F \in \operatorname{Lin}\left(c_{0}(\alpha, \mathbf{K})\right)$, then $[W(F)]_{i, j}=F_{j, i}$ for all $i, j \in \alpha$. Taking $\epsilon_{n}=p^{-n}, n \in \mathbf{N}$, gives that $x$ has non-zero entries only in a countable subset $\beta(x) \subset \alpha$ and there exists $\lim _{j \in \alpha} x_{j}=0$. If $a \in X$ and $b \in X$, then $(a, b)=\sum_{j \in \alpha} a_{j} b_{j}$ converges due to the non-archimedean inequality for the norm and $\lim _{j \in \alpha} a_{j} b_{j}=0$. If $a, b \in X$, then $[a, b]=F$ with $F_{l, j}=a_{l} b_{j}$ for all $l, j \in \alpha$, consequently, $F \in \operatorname{Lc}\left(c_{0}(\alpha, \mathbf{K})\right)$.

If the field $\mathbf{K}$ is spherically complete, then a Banach space over $\mathbf{K}$ is isomorphic with $c_{0}(\alpha, \mathbf{K})$ for some set $\alpha$ and each closed $\mathbf{K}$-linear subspace $Z$ in $X$ is complemented (see Theorems 5.13 and 5.16 [37]). Then certain closed $\mathbf{K}$-linear subspaces of products of Banach spaces $c_{0}(\alpha, \mathbf{K})$ can serve as further examples.

32. Note. Henceforth, we shall suppose that

$(D)$ a complete $\mathbf{K}$-convex space $X$ (see $\S 31)$ has an everywhere dense linear subspace $X_{0}$ isomorphic with $c_{0}(\alpha, \mathbf{K})$ such that a topology $\tau_{0}$ in $X_{0}$ inherited from the topology $\tau$ in $X$ is weaker or equal to that of the norm topology $\tau_{c}$ in $c_{0}(\alpha, \mathbf{K})$.

In the particular case of $\tau_{0}=\tau_{c}$ we can take $X=c_{0}(\alpha, \mathbf{K})$.

33. Lemma. Let $X$ be a complete locally $\mathbf{K}$-convex space satisfying Condition 32(D). Then there exists a continuous linear mapping $\operatorname{Tr}: \operatorname{Lc}(X) \mapsto$ K.

Proof. Consider an arbitrary $F \in L c(X)$ and a semi-norm $u$ in $X$. If a finite-dimensional over $\mathbf{K}$ subspace $X_{\epsilon}$ is complemented in $X$, then there exists $X_{0, \epsilon}:=X_{0} \cap X_{\epsilon}, X_{0} \ominus X_{\epsilon}=X_{0} \cap Z_{\epsilon}=: Z_{0, \epsilon}$, where $Z_{\epsilon}=X \ominus X_{\epsilon}$ and $X_{0, \epsilon} \cap Z_{0, \epsilon}=\{0\}$, since $X_{\epsilon} \cap Z_{\epsilon}=\{0\}$. Thus $X_{0}=X_{0, \epsilon} \oplus Z_{0, \epsilon}$. 
Hence there exists the continuous compact restriction of $F$ on $X_{0}$. Then for each $\epsilon>0$ there exists a finite-dimensional over $\mathbf{K}$ subspace $X_{0, \epsilon}$ in $X_{0}$ such that $u(F x) \leq \epsilon u(x)$ for each $x \in Z_{0, \epsilon}$ and each semi-norm $u$ in $X_{0}$. Therefore,

(1) $\lim _{i \in \alpha} \sup _{j \in \alpha}\left|F_{i, j}\right|=0$,

since the family of semi-norms $\{u\}$ separates points in $X$, where $\left\{e_{j}: j \in \alpha\right\}$ is the basis in $X_{0}$ inherited from $c_{0}(\alpha, \mathbf{K})$ and by the Zermelo theorem we take as $\alpha$ an ordinal (see Example 31.1). Consequently, $\sum_{j \in \alpha} F_{j, j}$ converges in $\mathbf{K}$, since $\mathbf{K}$ is complete relative to its non-archimedean norm. Put

(2) $\left.\operatorname{Tr} F\right|_{X_{0}}:=\sum_{j \in \alpha} F_{j, j}$.

The space $X_{0, \epsilon}$ is isomorphic with $\mathbf{K}^{\mathbf{m}}$ for some $m \in \mathbf{N}$, where the norm in $\mathbf{K}^{\mathbf{m}}$ is equivalent to that of inherited from $c_{0}(\alpha, \mathbf{K})$. Then each basic vector $v_{k}$ in $X_{0, \epsilon}$ has an expansion over $\mathbf{K}$ by the basis $\left\{e_{j}: j \in \alpha\right\}$, consequently, for each $\delta>0$ there exists a finite subset $\beta$ in $\alpha$ such that $\left\|v_{k}-y_{k}\right\|_{c_{0}}<\delta$ for each $k$, where $y_{k} \in \operatorname{span}_{\mathbf{K}}\left\{e_{j}: j \in \beta\right\}$.

Thus for a suitable finite subset $\beta$ in $\alpha$ for each $x \in X_{0, \epsilon}$ there exists $y \in \operatorname{span}_{\mathbf{K}}\left\{e_{j}: j \in \beta\right\}$ such that $u(x-y) \leq \epsilon u(x)$, since $0 \leq u(a x+$ $b y) \leq \max (|a| u(x),|b| u(y))$ for each $x, y \in X$ and $a, b \in \mathbf{K}$. Therefore, $\operatorname{Tr}: \operatorname{Lc}\left(X_{0}\right) \rightarrow \mathbf{K}$ is the continuous $\mathbf{K}$-linear mapping relative to the topology $\tau_{0}$ in $X_{0}$ provided by the family of semi-norms $\{u\}$ and inevitably $\operatorname{Tr}$ has the continuous $\mathbf{K}$-linear extension on the completion $X$ of $X_{0}$ relative to the locally $\mathbf{K}$-convex topology $\tau$ in $X$.

34. Corollary. Let the conditions of Lemma 33 be satisfied and $F \in$ $\operatorname{Lc}(X)$. Then $\left\|\left.F\right|_{X_{0}}\right\|_{c_{0}(\alpha, \mathbf{K})}<\infty$.

Proof. In view of 33(1) it follows that $\sup _{i, j \in \alpha}\left|F_{i, j}\right|<\infty$, but $\sup _{i, j \in \alpha}\left|F_{i, j}\right|=$ $\left\|\left.F\right|_{X_{0}}\right\|_{c_{0}(\alpha, \mathbf{K})}$.

35. Definition. Suppose that for each $A \in \mathcal{R}(G)$ there is a random vector $\xi(A) \in X$. Let it be satisfying the conditions:

$(M 1) \xi(A) \in Y, \xi(\emptyset)=0$, where $Y=L^{2}(\Omega, \mathcal{R}, P, X)$

$(M 2) \xi\left(A_{1} \cup A_{2}\right)=\xi\left(A_{1}\right)+\xi\left(A_{2}\right) \bmod (P)$ for each $A_{1}, A_{2} \in \mathcal{R}(G)$ with $A_{1} \cap A_{2}=\emptyset$;

(M3) $M\left[\xi\left(A_{1}\right), \xi\left(A_{2}\right)\right]=\mu\left(A_{1} \cap A_{2}\right)$;

(M4) $M\left[\xi\left(A_{1}\right), \xi\left(A_{2}\right)\right]=0$ for each $A_{1} \cap A_{2}=\emptyset, A_{1}, A_{2} \in \mathcal{R}(G)$, that is $\xi\left(A_{1}\right)$ and $\xi\left(A_{2}\right)$ are orthogonal random variables, where $\mu(A) \in L c(X)$ for each $A, A_{1}, A_{2} \in \mathcal{R}(G)$.

The family of random vectors $\{\xi(A): A \in \mathcal{R}(G)\}$ satisfying Conditions $(M 1-M 4)$ we shall call the (elementary) orthogonal $X$-valued stochastic 
measure, the compact operator $\mu(A)$ is called the structural operator.

36. Lemma. If $A_{1}, A_{2} \in \mathcal{R}(G), A_{1} \cap A_{2}=\emptyset$, then $\mu\left(A_{1} \cup A_{2}\right)=$ $\mu\left(A_{1}\right)+\mu\left(A_{2}\right)$.

Proof. Generalizing the proof of Lemma 10 we get the statement of this lemma, since the product in $X$ with values in $L c(X)$ is continuous and $L c(X)$ is the locally $\mathbf{K}$-convex space having also the structure of the algebra over $\mathbf{K}$, while $L^{1}(P, X) \subset L^{2}(P, X)$ :

$\mu\left(A_{1} \cup A_{2}\right)=M\left[\xi\left(A_{1} \cup A_{2}\right), \xi\left(A_{1} \cup A_{2}\right)\right]=M\left[\xi\left(A_{1}\right)+\xi\left(A_{2}\right), \xi\left(A_{1}\right)+\right.$ $\left.\xi\left(A_{2}\right)\right]=M\left[\xi\left(A_{1}\right), \xi\left(A_{1}\right)\right]+M\left[\xi\left(A_{2}\right), \xi\left(A_{2}\right)\right]=\mu\left(A_{1}\right)+\mu\left(A_{2}\right)$, since

$M\left[\xi\left(A_{1}\right), \xi\left(A_{2}\right)\right]=0$ and $M\left[\xi\left(A_{2}\right), \xi\left(A_{1}\right)\right]=0$ for $A_{1} \cap A_{2}=\emptyset$.

37. Note. Generalize Definitions 1 . Let $Z$ be a locally $\mathbf{K}$-convex space with a family of semi-norms $\mathcal{S}(Z)$ defining its topology. If $\mathcal{A}$ is a shrinking family, $f: \mathcal{R} \rightarrow Z$, then we shall write $\lim _{A \in \mathcal{A}} f(A)=0$, if for each $\epsilon>0$ and each $u \in \mathcal{S}(Z)$ there exists $A_{0} \in \mathcal{A}$ such that $u(f(A))<\epsilon$ for each $A \in \mathcal{A}$ with $A \subset A_{0}$.

A measure $\mu: \mathcal{R} \rightarrow Z$ is a mapping with values in $Z$ satisfying the following properties:

(i) $\mu$ is additive;

(ii) for each $A \in \mathcal{R}$ the set $\{\mu(B): B \in \mathcal{R}, A \subset B\}$ is bounded;

(iii) if $\mathcal{A}$ is the shrinking family in $\mathcal{R}$ and $\bigcap_{A \in \mathcal{A}} A=\emptyset$, then $\lim _{A \in \mathcal{A}} \mu(A)=$ 0 .

Henceforth, we suppose that $\mu$ has an extension to a $L c(X)$-valued measure on the separating covering ring $\mathcal{R}(G)=\mathcal{R}$.

38. Lemma. If $X$ is a complete locally $\mathbf{K}$-convex space satisfying Condition 32(D) and $\mu$ is as in $\S 37$, then there exists the trace $\operatorname{Tr} \mu(A)$ of $\mu$ for each $A \in \mathcal{R}(G)$. Moreover, $\operatorname{Tr} \mu$ is the $\mathbf{K}$-valued measure.

Proof. In view of Lemma 33 there exists the continuous mapping $\mathrm{Tr}$ : $L c(X) \rightarrow \mathbf{K}$, hence $\operatorname{Tr} \mu(A) \in \mathbf{K}$ for each $A \in \mathcal{R}(G)$, since $\mu(A)$ is the compact operator. Then $\|\mu(A)\|_{u}:=\sup _{u(x) \neq 0, x \in X}, u(\mu(A) x) / u(x) \leq \sup _{i, j \in \alpha}\left|[\mu(A)]_{i, j}\right|=$ $\left\|\left.[\mu(A)]\right|_{X_{0}}\right\|_{c_{0}}<\infty$ due to Corollary 34 , consequently,

(1) $|\operatorname{Tr} \mu(A)| \leq\left\|\left.[\mu(A)]\right|_{X_{0}}\right\|_{c_{0}}$ for every $A \in \mathcal{R}(G)$. Therefore, if $\mathcal{A}$ is the shrinking family in $\mathcal{R}$ and $\bigcap_{A \in \mathcal{A}} A=\emptyset$, then $\lim _{A \in \mathcal{A}} \mu(A)=0$, hence $\lim _{A \in \mathcal{A}}\|\mu(A)\|_{u}=0$ for each semi-norm $u$ in $X$ and inevitably $\lim _{A \in \mathcal{A}} \operatorname{Tr} \mu(A)=0$ due to Inequality (1) and $\mu(A) \in L c(X)$ and 33(1).

39. Definition. Let $\mathrm{g}$ be a complete locally $\mathbf{K}$-convex algebra with a unit 1 and $X$ be a complete locally $\mathbf{K}$-convex space satisfying Condition $32(D)$ 
and let simultaneously $X$ be a unital left g-module, where $g$ also satisfies Conditions $31(T 1, T 2)$. This means, that there exists a mapping $\mathrm{g} \times X \rightarrow X$ satisfying conditions $(1-5)$ :

(1) $b\left(x_{1}+x_{2}\right)=b x_{1}+b x_{2}$,

(2) $\left(b_{1}+b_{2}\right) x=b_{1} x+b_{2} x$,

(3) $b_{1}\left(b_{2} x\right)=\left(b_{1} b_{2}\right) x$

(4) $1 x=x$,

(5) there exists a family of consistent semi-norms $\mathcal{S}=\{u\}$ in $\mathrm{g}$ and $X$ defining their Hausdorff topologies such that $u(b x) \leq u(b) u(x), u(a b) \leq$ $u(a) u(b)$,

(6) $(a x, b y)=\left(b^{T} a x, y\right)=\left(x, a^{T} b y\right) \in \mathbf{K}$ and

(7) $[a x, b y]=[a, b][x, y] \in L c(X)$ such that $L c(X)$ is the left $L c(\mathrm{~g})$-module for each $a, b, b_{1}, b_{2} \in \mathrm{g}$ and every $x, y, x_{1}, x_{2} \in X$.

For each $f \in L^{0}(\mathcal{R}, \mathrm{g})$ define the stochastic integral:

$(S I) \eta=\int_{G} f(x) \xi(d x):=\sum_{k} a_{k} \xi\left(A_{k}\right)$,

where $f(x)=\sum_{k} a_{k} C h_{A_{k}}(x), a_{k} \in \mathrm{g}$.

By $L^{0}(\xi, \mathrm{g})=L^{0}(\xi)$ denote the family of all random vectors $\eta$ of the form $(S I)$.

40. Examples. Consider either $\mathbf{g}=\mathbf{K}$ or a subalgebra $\mathbf{g}$ in $\operatorname{Lin}(X)$ and $X$ is a complete locally $\mathbf{K}$-convex space, each semi-norm $v$ in $X$ induces the consistent semi-norm $\|F\|_{v}:=\sup _{x \in X, v(x) \neq 0} v(F x) / v(x)$ for each $F \in L(X)$. For simplicity of the notation we can denote $\|F\|_{v}$ also by $v(F)$ and these semi-norms in $X$ and in $\operatorname{Lin}(X)$ are consistent, since $v(F x) \leq\|F\|_{v} v(x)$, where we distinguish $v(F)$ and $v(F x)$, each multiple $b I$ of the unit operator $I$ also belongs to $\operatorname{Lin}(X)$ for $b \in \mathbf{K}$.

Take now a group $H$ with a $\mathbf{K}$-valued measure $\nu$ on $\mathcal{R}(H)$ or particularly $\nu$ on $\mathrm{B}_{\mathrm{c}}(H)$ and $H$ may be a topological totally disconnected group such that $\sup _{x \in H} N_{\nu}(x)=1$. Let $L_{b}^{q}\left(H, \mathrm{~B}_{\mathrm{c}}(H), \nu, \mathrm{b}\right)$ be a completion of the family of all step functions $f: H \rightarrow \mathrm{b}$ with supports in $A \in \mathrm{B}_{\mathrm{c}}(H)$ on which $\left.\nu\right|_{A}$ is the measure relative to the family of all non-archimedean semi-norms $\|f\|_{q, b, v}=\left[\sup _{x \in H, y \in H} v\left[f\left(y^{-1} x\right)\right]^{q} N_{\nu}(x)\right]^{1 / q}<\infty$, where $1 \leq q<\infty$, b is a complete locally convex algebra over $\mathbf{K}$ with a family of semi-norms $\{v\}$ in it and $\mathrm{b}$ satisfies Conditions $31(T 1, T 2)$. Certainly $v(x y) \leq v(x) v(y)$ for each $x, y \in \mathrm{b}$ and every semi-norm $v$. In particular, this space is defined for the measure $\nu$ on $\mathcal{R}(H)$.

If $f_{1}, f_{2} \in L_{b}^{1}(H, \nu, \mathrm{b})$, then define the convolutions

(1) $\operatorname{conv}\left\{f_{1}, f_{2}\right\}:=\left\{f_{1} * f_{2}\right\}(x):=\int_{H} f_{1}\left(y^{-1} x\right) f_{2}(x) \nu(d x)$ and 
(2) $\operatorname{conv}\left[f_{1}, f_{2}\right]:=\left[f_{1} * f_{2}\right](x):=\int_{H}\left[f_{1}\left(y^{-1} x\right), f_{2}(x)\right] \nu(d x)$ and

(3) $\operatorname{conv}\left(f_{1}, f_{2}\right):=\left(f_{1} * f_{2}\right)(x):=\int_{H}\left(f_{1}\left(y^{-1} x\right), f_{2}(x)\right) \nu(d x)$.

They are defined for simple functions. If they exist then

(4) $\sup _{x \in H, z \in H} v\left[\left\{f_{1} * f_{2}\right\}\left(z^{-1} x\right)\right] N_{\nu}(x) \leq$

$\sup _{x \in H, y \in H, z \in H} v\left[f_{1}\left(y^{-1} z^{-1} x\right)\right] v\left[f_{2}\left(z^{-1} x\right)\right] N_{\nu}(x) N_{\nu}\left(z^{-1} x\right) \leq\left\|f_{1}\right\|_{1, b, v}\left\|f_{2}\right\|_{1, b, v}<$ $\infty$ and

(5) $\sup _{x \in H, z \in H} v\left[\left[f_{1} * f_{2}\right]\left(z^{-1} x\right)\right] N_{\nu}(x) \leq$

$\sup _{x \in H, y \in H, z \in H} C_{v} v\left[f_{1}\left(y^{-1} z^{-1} x\right)\right] v\left[f_{2}\left(z^{-1} x\right)\right] N_{\nu}(x) N_{\nu}\left(z^{-1} x\right) \leq C_{v}\left\|f_{1}\right\|_{1, b, v}\left\|f_{2}\right\|_{1, b, v}<$ $\infty$ and

(6) $\sup _{x \in H, z \in H}\left|\left(f_{1} * f_{2}\right)\left(z^{-1} x\right)\right| N_{\nu}(x) \leq$

$\sup _{x \in H, y \in H, z \in H} J_{v} v\left[f_{1}\left(y^{-1} z^{-1} x\right)\right] v\left[f_{2}\left(z^{-1} x\right)\right] N_{\nu}(x) N_{\nu}\left(z^{-1} x\right) \leq J_{v}\left\|f_{1}\right\|_{1, b, v}\left\|f_{2}\right\|_{1, b, v}<$

$\infty$,

since the mappings $31(T 1, T 2)$ are continuous, where the semi-norm in $\operatorname{Lin}(\mathrm{b})$

induced by the semi-norm $v$ in b is also denoted by $v, J_{v}$ and $C_{v}$ are finite semi-norms of the mappings $31(T 1, T 2)$ correspondingly relative to the seminorm $v$ in $\mathrm{g}$.

Therefore, the convolutions have the continuous extensions on $L_{b}^{1}(H, \nu, \mathrm{b})=$ :

$X$ such that $\operatorname{conv}\left\{f_{1}, f_{2}\right\} \in L_{b}^{1}(H, \nu, \mathrm{b}), \operatorname{conv}\left[f_{1}, f_{2}\right] \in L_{b}^{1}(H, \nu, L c(\mathbf{b})), \operatorname{conv}\left(f_{1}, f_{2}\right) \in$ $L_{b}^{1}(H, \nu, \mathbf{K})$. The space $X$ is $\mathbf{K}$-linear and complete and it is the algebra with the multiplication being the convolution $\operatorname{conv}\left\{f_{1}, f_{2}\right\}$. If 1 is not in this space adjoin it and we get the complete locally $\mathbf{K}$-convex algebra with the unit $1(x)=1$ for each $x \in H$.

This is the group algebra $X$ of $H$ over b. Particularly, we can take $\mathrm{b}=\operatorname{Mat}_{m}(\mathbf{K})$ also or more general algebras as above. Then the transposition in b induces it in $X=L_{b}^{1}(H, \nu, \mathrm{b})$ such that

$\left(f_{1}, f_{2}\right):=\int_{H}\left(f_{1}(x), f_{2}(x)\right) \nu(d x) \in \mathbf{K}$ and

$\left[f_{1}, f_{2}\right]:=\left[f_{1} * f_{2}\right] \in \operatorname{Lin}(X)$ can be considered as the linear operator $F$ on $X$ such that $F \in \operatorname{Lin}(X)$,

(7) $F f(x)=<\left[f_{1} * f_{2}\right] * f>(x)$, where

(8) $<g * f>(x):=\int_{H} g\left(y^{-1} x\right) f(x) \nu(d x)$

for each $g \in L_{b}^{1}(H, \mathcal{R}, \nu, \operatorname{Lin}(\mathrm{b}))$ and each $f \in X$ and every $x \in H$. If $\mathrm{b}$ satisfies Condition 39(1-7), then $X$ also satisfies 39(1-7). If $\mathrm{b}$ is the Banach algebra, then $X$ also is the Banach algebra.

41. Theorem. Let $F f(x)=<\left[f_{1} * f_{2}\right] * f>(x)$, where $f, f_{1}, f_{2} \in$ $L_{b}^{1}(H, \nu, \mathrm{b})=X$ and $H$ is the topological group, $\mathcal{R}(H) \subset \operatorname{Bco}(H)$ as in Example 40. Then $F$ is the compact operator $F \in L c(X)$ and the mapping $X^{2} \ni\left\{f_{1}, f_{2}\right\} \mapsto<\left[f_{1} * f_{2}\right] *>\in L c(X)$ is continuous. 
Proof. It was demonstrated in Example 40, that the convolution conv $\left[f_{1}, f_{2}\right]$ is continuous from $X^{2}$ into $L_{b}^{1}(H, \nu, L c(\mathbf{b}))$, where $\nu$ is the $\mathbf{K}$-valued measure. The space $L_{b}^{1}(H, \nu, L c(\mathrm{~b}))$ is the completion of the family of all step functions $g(x)=\sum_{k} C h_{A_{k}}(x) a_{k}$ relative to the family of semi-norms $\|g\|_{1, b, v}=$ $\left[\sup _{x \in H, y \in H} v\left[g\left(y^{-1} x\right)\right] N_{\nu}(x)\right]<\infty$, where $\|Y\|_{v, \mathrm{~b}}:=\sup _{v(t) \neq 0, t \in \mathrm{b}} v(Y t) / v(t)$ is the semi-norm in $\operatorname{Lin}(\mathrm{b})$ denoted also by $v(Y), A_{k} \in \mathcal{R}(H), a_{k} \in L c(\mathrm{~b})$. Therefore, it is sufficient to demonstrate that $F \in L c\left(X_{s}\right)$ and the mapping $X_{s}^{2} \ni\left\{f_{1}, f_{2}\right\} \mapsto\left[f_{1} * f_{2}\right] \in L c\left(X_{s}\right)$ is continuous, where $X_{s}:=L_{b}^{1}(H, \nu, \mathbf{K})$.

Theorem 7.12 [37] states, that $f \in L(H, \mathcal{R}, \nu, \mathbf{K})$ if and only if it has two properties: $(i) f$ is $\mathcal{R}_{\nu}$-continuous, $(i i)$ for every $\epsilon>0$ the set $\{x$ : $\left.|f(x)| N_{\nu}(x) \geq \epsilon\right\}$ is $\mathcal{R}_{\nu}$-compact, hence contained in $\left\{x: N_{\nu}(x) \geq \delta\right\}$ for some $\delta>0$. For vector valued functions see Theorem 56 below, which is proved independently from $\S \S 40,41$. Thus if $f_{1}, f_{2} \in X_{s}$, then $f_{1} * f_{2}$ is $\mathcal{R}_{\nu}$-continuous and for each $\epsilon>0$ and every semi-norm $v$ in $\mathrm{b}$ there exists $\delta>0$ such that $\left\{x: v\left(f_{1} * f_{2}(x)\right) N_{\nu, v}(x) \geq \epsilon\right\} \subset\left\{x: N_{\nu, v}(x) \geq \delta\right\}$, where $\left\{x: v\left(f_{1} * f_{2}(x)\right) N_{\nu, v}(x) \geq \epsilon\right\}$ and $\left\{x: N_{\nu, v}(x) \geq \delta\right\}$ are $\mathcal{R}_{\nu}$-compact hence $\mathcal{R}$-compact sets.

If $f \in L(H, \mathcal{R}, \nu, \mathrm{b})$, then for each $\epsilon>0$ and each semi-norm $v$ in $\mathrm{b}$ and every $x \in H$ there exists an open symmetric neighborhood $U_{x}$ of the unit element $e$ in the topological group $H$ such that $v\left(f\left(y^{-1} x\right)-f(x)\right)<\epsilon$ for each $y \in U_{x}^{3}$. From the covering $\left\{x U_{x}: x \in H, N_{\nu, v}(x) \geq \delta\right\}$ of $\left\{x: N_{\nu, v}(x) \geq \delta\right\}$ extract a finite covering $\left\{x_{j} U_{x_{j}}: j=1, \ldots, q\right\}$ and take $U=\bigcap_{j=1}^{q} U_{x_{j}}$, since $\mathcal{R} \subset \operatorname{Bco}(H)$. Then $U$ is open symmetric $U^{-1}=U$ and $e \in U$.

If $y \in U$ and $N_{\nu, v}(x) \geq \delta$, then there exists $j$ such that $x \in x_{j} U_{x_{j}}$, hence $v\left(f\left(y^{-1} x\right)-f(x)\right) \leq \max \left(v\left(f\left(y^{-1} x\right)-f\left(y^{-1} x_{j}\right)\right), v\left(f\left(y^{-1} x_{j}\right)-f\left(x_{j}\right)\right), v\left(f\left(x_{j}\right)-\right.\right.$ $f(x)))<\epsilon$, since $\left(y^{-1} x\right)\left(y^{-1} x_{j}\right)^{-1} \in U^{3} \subset U_{x_{j}}^{3}$. Consequently, $v\left(f\left(y^{-1} z\right)-\right.$ $f(z)) \leq \max \left(v\left(f\left(y^{-1} z\right)-f\left(y^{-1} t\right)\right), v\left(f\left(y^{-1} t\right)-f(t)\right)\right)<\epsilon$ for each $z \in$ $\left[U\left\{x \in H: N_{\nu, v}(x) \geq \delta\right\}\right]$, where $t \in\left\{x \in H: N_{\nu, v}(x) \geq \delta\right\}$ is such that $z t^{-1} \in U$, since $\left(y^{-1} z\right)\left(y^{-1} t\right)^{-1} \in U^{3}$. At the same time $\{x \in H$ : $\left.N_{\nu, v}(x) \geq \delta\right\} \subset\left[U\left\{x \in H: N_{\nu, v}(x) \geq \delta\right\}\right]$ and $v(f(z)) N_{\nu, v}(z)<\epsilon$ for each $z \in H \backslash\left\{x \in H: N_{\nu, v}(x) \geq \delta\right\}$. If $z \in H \backslash\left[U\left\{x \in H: N_{\nu, v}(x) \geq \delta\right\}\right]$, then $U z \cap\left\{x \in H: N_{\nu, v}(x) \geq \delta\right\}=\emptyset$. Thus for each $f \in L(H, \mathcal{R}, \nu, \mathrm{b})$ and every $\epsilon>0$ and each semi-norm $v$ in b there exists an open symmetric neighborhood $U$ of $e$ in $H$ such that $v\left(f\left(y^{-1} x\right)-f(x)\right)<\epsilon$ for each $x \in H$ and $y \in U$.

Suppose that $f, f_{1}, f_{2} \in X_{s}$, then for each $\epsilon>0$ there exists an open 
symmetric neighborhood $U$ of $e$ in $H$ such that

(iii) $v\left(<\left[f_{1} * f_{2}\right] * f>\left(y^{-1} x\right)-<\left[f_{1} * f_{2}\right] * f>(x)\right) N_{\nu, v}(x)<\epsilon \sup _{x \in H} v(f(x)) N_{\nu, v}(x)$ for each $x \in H$ and every $y \in U$. Take the sets $\left\{x: v\left(\left[f_{1} * f_{2}\right](x)\right) N_{\nu, v}(x) \geq\right.$ $\epsilon\} \subset\left\{x: N_{\nu, v}(x) \geq \delta\right\}=: A$ for $\left[f_{1} * f_{2}\right]$ as above. Decompose $F=F_{1}+F_{2}$, where $F_{1} f:=<\left[f_{1} * f_{2}\right] *\left(C h_{A} f\right)>$ and $F_{2} f:=<\left[f_{1} * f_{2}\right] *\left(\left(1-C h_{A}\right) f>\right.$. Then $\left\|F_{2}\right\|_{v} \leq \epsilon$.

In view of Inequality (iii) for each $\epsilon>0$ there exists a finite covering family of subsets $A_{1}, \ldots, A_{m} \in \mathcal{R}$ in $A, \cup_{j=1}^{m} A_{j}=A$, such that for each $f \in X_{s}$ there exists a simple function $g(x)=\sum_{j=1}^{m} b_{k} C h_{A_{k}}(x)$ for which $\| F_{1}(f-$ $g) \|_{v}<\epsilon$. But the family of such simple functions $g$ is finite-dimensional over $\mathbf{K}$, consequently, $F$ is the compact operator.

In view of $40(2,5)$ the mapping $X^{2} \ni\left\{f_{1}, f_{2}\right\} \mapsto<\left[f_{1} * f_{2}\right] *>\in L c(X)$ is continuous.

42. Lemma. Let for a natural number $k$ there exists $M \xi^{k}$, where $\xi$ is a random vector with values in a locally $\mathbf{K}$-convex algebra $\mathbf{g}$, then for each $1 \leq l<k$ there exists $M \xi^{l}$.

Proof. If $\xi$ is a random vector with values in $\mathrm{g}$, then $\xi$ by the definition it is $(\mathcal{A}, \mathcal{R}(\mathrm{g}))$-measurable and for each semi-norm $u$ in $\mathrm{g}$ there exists $\sup _{\omega \in \Omega}\left[u(\xi)^{k} N_{P}(\omega)\right]^{1 / k}=\|\xi\|_{k, u}<\infty$, where $\mathcal{R}(\mathrm{g})$ is a separating covering ring of $\mathrm{g}$ such that $\mathcal{R}(\mathrm{g}) \subset \mathrm{Bco}(\mathrm{g})$. Therefore, $\sup _{\omega \in \Omega}\left[u(\xi)^{l} N_{P}(\omega)\right]^{1 / l} \leq$ $\sup _{\omega \in \Omega}\left[u(\xi)^{k} N_{P}(\omega)\right]^{1 / k}$ for each $1 \leq l<k$, since $N_{P}(\omega) \leq 1$ for each $\omega \in \Omega$.

43. Remark. Denote by $L^{2}(\xi, \mathrm{g})$ the completion of $L^{0}(\xi, \mathrm{g})$ by the family of semi-norms

(1) $\|f\|_{2, P, u}:=\left[\sup _{x \in G, \omega \in \Omega} u^{2}(f(x) \xi(\omega, x)) N_{P}(\omega)\right]^{1 / 2}$ induced from $L^{2}(\Omega, \mathcal{A}, P, X)$, where $L^{0}(\xi, \mathrm{g})$ is the space of all step functions $\sum_{k=1}^{l} a_{k} \xi\left(A_{k}\right), a_{k} \in \mathrm{g}, A_{k} \in \mathcal{R}(G), A_{j} \cap A_{k}=\emptyset$ for each $k \neq j, l \in \mathbf{N}$.

44. Definition. Suppose that $Z$ is a locally $\mathbf{K}$-convex space and $\mu$ : $\mathcal{R} \rightarrow Z$ is a measure, where $\mathcal{R}=\mathcal{R}(G)$ is a separating covering ring of a set $G$. For each semi-norm $u$ in $Z$ and any $A \in \mathcal{R}(G)$ define

(1) $\|A\|_{\mu, u}:=\sup \{u(\mu(B)): B \in \mathcal{R}, B \subset A\}$.

45. Lemma. If $\mu: \mathcal{R} \rightarrow Z$ is a measure, then for each semi-norm $u$ in $Z$ there exists a unique function $N_{\mu, u}: G \rightarrow[0, \infty)$ such that

(1) $\left\|C h_{A}\right\|_{N_{\mu, u}}=\|A\|_{\mu, u}$ for each $A \in \mathcal{R}$ (see Definition 44);

(2) if $\phi: G \rightarrow[0, \infty)$ and $\left\|C h_{A}\right\|_{\phi} \leq\|A\|_{\mu, u}$ for all $A \in \mathcal{R}$, then $\phi \leq N_{\mu, u}$; moreover,

(3) $N_{\mu, u}(x)=\inf _{A: x \in A \in \mathcal{R}}\|A\|_{\mu, u}$ for each $x \in G$. 
Proof. If $N_{\mu, u}(x)$ is defined by the formula (3), then (2) is evident, since $\left\|C h_{A}\right\|_{\phi} \leq\|A\|_{\mu, u}$ for all $A \in \mathcal{R}$. Take $A \in \mathcal{R}$ and consider the family $\mathcal{E}:=\left\{B \in \mathcal{R}: B \subset A,\|A \backslash B\|_{\mu, u} \leq\left\|C h_{A}\right\|_{N_{\mu, u}}+\epsilon\right\}$.

On the other hand, from 44(1) it follows that $\left\|A_{1} \cup A_{2}\right\|_{\mu, u} \leq \max \left(\left\|A_{1}\right\|_{\mu, u},\left\|A_{2}\right\|_{\mu, u}\right\}$ for each $A_{1}, A_{2} \in \mathcal{R}$, since $\mu$ is additive and for each $B \subset A_{1} \cup A_{2}$ we have $B=B_{1} \cup B_{2}$, while $u\left(\mu\left(A_{1} \cup A_{2}\right)\right) \leq \max \left\{u\left(\mu\left(A_{1} \backslash A_{2}\right)\right), u\left(\mu\left(A_{2} \backslash\right.\right.\right.$ $\left.\left.\left.A_{1}\right)\right), u\left(\mu\left(A_{1} \cap A_{2}\right)\right)\right\}$, where $B_{1}:=A_{1} \cap B$ and $B_{2}:=A_{2} \cap B$. Therefore, $\mathcal{E}$ is the shrinking family.

Then for each $x \in A$ there exists $B \in \mathcal{R}$ such that $x \in B$ and $\|B\|_{\mu, u} \leq$ $N_{\mu, u}(x)+\epsilon \leq\left\|C h_{B}\right\|_{N_{\mu, u}}+\epsilon$, consequently, $A \backslash B \in \mathcal{E}$. Then $\bigcap_{A \in \mathcal{E}} A=$ $\emptyset$, consequently, there exists $B \in \mathcal{E}$ such that $\|B\|_{\mu, u} \leq \epsilon$ and inevitably $\|A\|_{\mu, u} \leq \max \left\{\|B\|_{\mu, u},\|A \backslash B\|_{\mu, u}+\epsilon\right\}$.

46. Definitions. Let $\mu$ be a $X$-valued measure as in $\S \S 37,39$. For $f: G \rightarrow \mathrm{g}$ consider the family of semi-norms

$\|f\|_{q, \mu, u}:=\left[\sup _{x \in G} u^{q}(f) N_{\mu, u}(x)\right]^{1 / q}$

whenever it exists, where $1 \leq q<\infty$ and $u$ is a semi-norm in $X$. Define the space $L^{q}(G, \mathcal{R}, \mu, X ; \mathrm{g})$ as the completion of the family of all step (simple) functions $f$ relative to the family of semi-norms $\left\{\|*\|_{q, \mu, u}: u \in \mathcal{S}\right\}$. If $f \in L^{1}(G, \mathcal{R}, \mu, X ; \mathrm{g})$, then it is called $\mu$-integrable.

In the particular case of $\mathbf{g}=\mathbf{K}$ we can omit it from the notation. When $G, \mathcal{R}, \mu, X$ and $\mathrm{g}$ are specified it also can be written shortly $L^{q}(\mu)$, for $q=1$ it can be omitted writing $L(\mu)$.

A function $f$ is called $\mu$-integrable, if $f \in L(\mu)$. Put $\mathcal{R}_{\mu}:=\{A \subset$ $\left.G: C h_{A} \in L(\mu)\right\}$ for the $X$-valued measure $\mu$ and extend it by $\bar{\mu}(A):=$ $\int_{G} C h_{A}(x) \mu(d x)$, since $1 \in \mathrm{g}$.

47. Lemma. If $\mu$ and $\mathcal{R}_{\mu}$ are as in $\S 46$, then $A \in \mathcal{R}_{\mu}$ if and only if for each $\epsilon>0$ and each semi-norm $u$ in $X$ there exists $B \in \mathcal{R}$ such that $N_{\mu, u}(x) \leq \epsilon$ for every $x \in A \triangle B$.

Proof. If for each $\epsilon>0$ and a semi-norm $u$ in $X$ there exists $B \in \mathcal{R}$ such that $\left\|C h_{A}-C h_{B}\right\|_{N_{\mu, u}}<\epsilon$, then taking a sequence $\epsilon_{n}=p^{-n}$ we get, that $C h_{A} \in L(\mu)$, hence $A \in \mathcal{R}_{\mu}$.

On the other hand, if $A \in \mathcal{R}_{\mu}$, then for each $1>\epsilon>0$ there exists a simple function $f \in L(\mu)$ such that $\left\|C h_{A}-f\right\|_{N_{\mu, u}}<\epsilon$. Consider $B:=$ $\{x: u(f(x)-1)<1\}$ which is in $\mathcal{R}, B \in \mathcal{R}$. Then $u\left(f(x)-C h_{B}(x)\right) \leq$ $\min [u(f(x)), u(f(x)-1)] \leq u\left(f(x)-C h_{A}(x)\right)$ for each $x \in G$ and inevitably $\left\|C h_{A}-C h_{B}\right\|_{N_{\mu, u}} \leq \max \left[\left\|f-C h_{A}\right\|_{N_{\mu, u}},\left\|f-C h_{B}\right\|_{N_{\mu, u}}\right]=\left\|f-C h_{A}\right\|_{N_{\mu, u}} \leq \epsilon$.

48. Lemma. If $\mu: \mathcal{R} \rightarrow X$ satisfies Conditions $37(i, i i)$, then $37($ iii $)$ is 
equivalent to:

$($ iii $)$ if $\mathcal{A} \subset \mathcal{R}$ is a shrinking family and $\bigcap_{A \in \mathcal{A}} A=\emptyset$, then $\lim _{A \in \mathcal{A}}\|A\|_{\mu, u}=$ 0 for each semi-norm $u \in \mathcal{S}$ in $X$.

Proof. Since $\|A\|_{\mu, u} \geq u(\mu(A))$ for each $A \in \mathcal{R}$, then $\left(i i i^{\prime}\right)$ implies $37($ iii $)$.

Prove now the converse statement supposing that $\mu$ satisfies $37(i-i i i)$. Suppose that $\mathcal{A}$ is a shrinking family with $\bigcap_{A \in \mathcal{A}} A=\emptyset$. For each $\epsilon>0$ and every $u \in \mathcal{S}$ there exist $E \in \mathcal{A}$ such that $u(\mu(A))<\epsilon$ for each $A \in \mathcal{A}$ such that $A \subset E$. Take a semi-norm $u \in \mathcal{S}$ in $X$. For every $A \in \mathcal{A}$ choose $V_{A} \in \mathcal{R}$ such that $V_{A} \subset A$ and $u\left(\mu\left(V_{A}\right)\right)>\min \left(\epsilon,\|A\|_{\mu, u} / 2\right)$. If $A \in \mathcal{A}$, then the family $\mathcal{C}:=\left\{V_{A} \cap B: B \in \mathcal{A}, B \subset A\right\}$ is shrinking and $\bigcap_{U \in \mathcal{C}} U=\emptyset$, hence for each $A \in \mathcal{A}$ there exists $W_{A} \in \mathcal{A}$ such that $W_{A} \subset A$ and $u\left(\mu\left(V_{A} \cap W_{A}\right)\right)<\epsilon$.

Take the family $\mathcal{V}:=\left\{V_{A} \cup W_{A}: A \in \mathcal{A}, A \subset E\right\}$. If $A, B \in \mathcal{A}$, then there exists $C \in \mathcal{A}$ with $C \subset W_{A} \cap W_{B}$. Then $V_{C} \cup W_{C} \subset C \subset W_{A} \subset V_{A} \cup W_{A}$, also $V_{C} \cup W_{C} \subset V_{B} \cup W_{B}$, consequently, the family $\mathcal{V}$ is shrinking. Moreover, $\bigcap_{C \in \mathcal{V}} C=\emptyset$. Thus there exists $A \in \mathcal{A}$ with $A \subset E$ and $u\left(\mu\left(V_{A} \cup W_{A}\right)\right)<\epsilon$, also $u\left(\mu\left(V_{A} \cup W_{A}\right)\right)<\epsilon$ and $u\left(\mu\left(V_{A} \cap W_{A}\right)\right)<\epsilon$ due to the definition of $W_{A}$, as well as $u\left(\mu\left(W_{A}\right)\right)<\epsilon$, since $W_{A} \in \mathcal{A}$ and $W_{A} \subset A \subset E$. Hence $u\left(\mu\left(V_{A}\right)\right)=u\left(\mu\left(V_{A} \cup W_{A}\right)+\mu\left(V_{A} \cap W_{A}\right)-\mu\left(W_{A}\right)\right)<\epsilon$. Therefore, $\|A\|_{\mu, u} \leq 2 \epsilon$, since $u\left(\mu\left(V_{A}\right)\right)>\min \left(\epsilon,\|A\|_{\mu, u} / 2\right)$.

49. Theorem. Let $\mu$ be a $X$-valued measure on $\mathcal{R}$. Then $\mathcal{R}_{\mu}$ is a covering ring of $G$ and $\bar{\mu}$ is a $X$-valued measure extending $\mu$.

Proof. In view of Lemma $47 \mathcal{R}_{\mu}$ is a covering ring of $G$ and $\bar{\mu}$ is additive. If $A \in \mathcal{R}_{\mu}$, then for each $B \subset A$ such that $B \in \mathcal{R}$ and each semi-norm $u$ in $X$ the inequalities are satisfied:

$u(\bar{\mu}(B)) \leq\left\|C h_{B}\right\|_{N_{\mu, u}} \leq\left\|C h_{A}\right\|_{N_{\mu, u}}<\infty$, hence $\bar{\mu}$ has property $37(i i)$.

Consider now any shrinking family $\mathcal{A} \subset \mathcal{R}_{\mu}$ having empty intersection. For $\epsilon>0$ and a semi-norm $u$ in $X$ take $\mathcal{E}:=\{B \in \mathcal{R}: \exists A \in \mathcal{A}$ such that $\left.A \cap G_{\epsilon, u}=B \cap G_{\epsilon, u}\right\}$, where $G_{\epsilon, u}:=\left\{x \in G: N_{\mu, u}(x) \geq \epsilon\right\}$. Then $\mathcal{E}$ is shrinking. If $x \notin G_{\epsilon, u}$, then there exists $V \in \mathcal{R}$ such that $x \in V$ and $\epsilon>\|V\|_{\mu, u}$, hence $B \backslash V \in \mathcal{E}$ for each $B \in \mathcal{E}$, consequently, $V \cap\left(\bigcap_{B \in \mathcal{E}} B\right)=$ $\emptyset$ and inevitably $\bigcap_{B \in \mathcal{E}} B \subset G_{\epsilon, u}$. Then by the construction of $\mathcal{E}$ we get: $\bigcap_{B \in \mathcal{E}} B=\bigcap_{B \in \mathcal{E}} B \cap G_{\epsilon, u}=\bigcap_{A \in \mathcal{A}} A \cap G_{\epsilon, u}=\emptyset$.

In view of Lemma 48 there exists $B \in \mathcal{E}$ such that $\|B\|_{\mu, u}<\epsilon$, hence $B \cap G_{\epsilon, u}=\emptyset$. Then there exists $A \in \mathcal{A}$ such that $A \cap G_{\epsilon, u}=B \cap G_{\epsilon, u}=\emptyset$, consequently, $\|A\|_{\mu, u}<\epsilon$. Again by Lemma $48 \lim _{A \in \mathcal{A}} \bar{\mu}(A)=0$. Thus $\bar{\mu}$ is the $X$-valued measure. 
50. Lemma. If $\mu$ is a $X$-valued measure on $\mathcal{R}$, then $N_{\mu, u}=N_{\bar{\mu}, u}$ for each semi-norm $u$ in $X$. Therefore, $\|*\|_{N_{\mu, u}}=\|*\|_{N_{\bar{\mu}, u}}, L(\bar{\mu})=L(\mu)$, $\int_{G} f d \bar{\mu}=\int_{G} f d \mu, \mathcal{R}_{\bar{\mu}}=\mathcal{R}_{\mu}$.

Proof. Take a semi-norm $u$ in $X$ and a point $x \in G$ and a number $b>$ $N_{\mu, u}(x)$. Then there exists $A \in \mathcal{R} \subset \mathcal{R}_{\mu}$ such that $x \in A$ and $\|A\|_{N_{\bar{\mu}, u}} \leq b$. Then for every $B \in \mathcal{R}$ such that $B \subset A$ there are inequalities $u(\bar{\mu}(B)) \leq$ $\|B\|_{N_{\bar{\mu}, u}} \leq\|A\|_{N_{\bar{\mu}, u}} \leq b$, hence

(1) $\|A\|_{N_{\bar{\mu}, u}} \leq b$, consequently, $N_{\bar{\mu}, u}(x) \geq \inf \left\{\|A\|_{\bar{\mu}, u}: A \in \mathcal{R}_{\mu}, x \in A\right\}$.

Take now $0<d<N_{\mu, u}(x)$ and $A \in \mathcal{R}_{\mu}$ with $x \in A$. In view of Lemma 47 there exists $B \in \mathcal{R}$ such that $N_{\bar{\mu}, u}(y) \leq d$ for each $y \in A \triangle B$. Therefore, $\|B\|_{N_{\bar{\mu}, u}} \geq N_{\bar{\mu}, u}>d$, so $u(\mu(E))>d$ for some $E \in \mathcal{R}$ such that $E \subset B$. As $u(\mu(E)-\bar{\mu}(E \cap A))=u\left(\bar{\mu}(E \backslash A) \leq\|E \backslash A\|_{N_{\bar{\mu}, u}} \leq\|E \backslash B\|_{N_{\bar{\mu}, u}} \leq d<u(\mu(E))\right.$. Thus $u(\bar{\mu}(E \cap A))=u(\mu(E))$, consequently,

(2) $\|A\|_{\bar{\mu}, u} \geq u(\bar{\mu}(E \cap A))=u(\mu(E))>d$. Finally, from these two Inequalities $(1,2)$ the equality $N_{\bar{\mu}, u}(x)=N_{\mu, u}(x)$ for each $x \in G$ follows.

51. Theorem. (1). If $\mu$ is a measure on $\mathcal{R}$, then $N_{\mu, u}$ is upper semicontinuous for each semi-norm $u \in \mathcal{S}$ in $X$, hence it is $\mathcal{R}_{\mu}$-upper semicontinuous and for every $A \in \mathcal{R}_{\mu}$ and $\epsilon>0$ the set $\left\{x \in A: N_{\mu, u}(x) \geq \epsilon\right\}$ is $\mathcal{R}_{\mu}$-compact, hence it is $\mathcal{R}$-compact.

(2). Conversely, let $\mu: \mathcal{R} \rightarrow X$ satisfies $37(i)$ and let for every $u \in$ $\mathcal{S}$ there exist an $\mathcal{R}$-semi-continuous function $\phi_{u}: G \rightarrow[0, \infty)$ such that $u(\mu(A)) \leq \sup _{x \in A} \phi_{u}(x)$ for each $A \in \mathcal{R}$ and let the set $\left\{x \in A: \phi_{u}(x) \geq \epsilon\right\}$ is $\mathcal{R}$-compact for each $\epsilon>0$. Then $\mu$ is a $X$-valued measure and $N_{\mu, u}(x) \leq$ $\phi_{u}(x)$ for each $x \in G$ and each $u \in \mathcal{S}$.

Proof. (1). Put $G_{\epsilon, u}:=\left\{x \in G: N_{\mu, u}(x) \geq \epsilon\right\}$, where $\epsilon>0$. Then for each $x \in G \backslash G_{\epsilon, u}$ there exists $A \in \mathcal{R}$ such that $x \in A$ and $\|A\|_{\mu, u}<\epsilon$, hence $A \subset G \backslash G_{\epsilon, u}$ and inevitably $G_{\epsilon, u}$ is $\mathcal{R}$-closed and $N_{\mu, u}$ is $\mathcal{R}$-upper semi-continuous. Take now $A \in \mathcal{R}_{\mu}$ and a covering $\mathcal{V}$ of $A \cap G_{\epsilon, u}$ by elements of $\mathcal{R}_{\mu}$. Then the sets $A \backslash\left(V_{1} \cup \ldots \cup V_{n} \cup V\right)$, where $n \in \mathbf{N}, V_{1}, \ldots, V_{n} \in \mathcal{V}$, $V \in \mathcal{R}_{\mu}$ and $V \subset G \backslash G_{\epsilon, u}$, form a shrinking subfamily $\mathcal{A}$ in $\mathcal{R}_{\mu}$ with the empty intersection $\bigcap_{E \in \mathcal{A}} E=\emptyset$. In accordance with Property $48\left(i i i^{\prime}\right)$ of an $X$-valued measure there exist $V_{1}, \ldots, V_{n} \in \mathcal{V}$ and $V \subset G \backslash G_{\epsilon, u}$ such that $\left\|A \backslash\left(V_{1} \cup \ldots \cup V_{n} \cup V\right)\right\|_{\bar{\mu}, u}<\epsilon$, hence $A \backslash\left(V_{1} \cup \ldots \cup V_{n} \cup V\right) \subset G \backslash G_{\epsilon, u}$. Since $V \subset G \backslash G_{\epsilon, u}$, then $A \cap G_{\epsilon, u} \subset V_{1} \cup \ldots \cup V_{n}$. Thus $A \cap G_{\epsilon, u}$ is $\mathcal{R}_{\mu}$-compact.

(2). Each function $\phi_{u}$ is $\mathcal{R}$-upper semi-continuous and $\phi_{u}$ is bounded from above on each $\mathcal{R}$-compact set, also $\|A\|_{\mu, u} \leq\left\|C h_{A}\right\|_{\phi_{u}}$ for each $A \in \mathcal{R}$. Thus for each $u \in \mathcal{S}$ and every $A \in \mathcal{R}$ there is the inequality $\|A\|_{\mu, u}<\infty$, 
consequently, 37(ii) is satisfied.

Take $\epsilon>0$ and a shrinking subfamily $\mathcal{A}$ in $\mathcal{R}$ such that $\bigcap_{E \in \mathcal{A}}=\emptyset$. Then the sets $\left\{x \in A: \phi_{u}(x) \geq \epsilon\right\}$ form a family $\mathcal{E}$ of $\mathcal{R}$-compact sets closed under finite intersections and $\bigcap_{B \in \mathcal{E}} B=\emptyset$. Therefore, there exists $E \in \mathcal{A}$ such that $\left\{x \in E: \phi_{u}(x) \geq \epsilon\right\}=\emptyset$. Hence $E \subset\left\{x \in G: \phi_{u}(x)<\epsilon\right\}$ and inevitably $\|A\|_{\mu, u}<\epsilon$.

52. Corollary. If $\mu$ is a $X$-valued measure on $\mathcal{R}$ then for every $\epsilon>0$ and each semi-norm $u \in \mathcal{S}$ the set $\left\{x \in G: N_{\mu, u}(x) \geq \epsilon\right\}$ is $\mathcal{R}$-locally compact.

53. Theorem. Let $\mu$ be a measure on $\mathcal{R}$ and let also $\mathcal{U}$ be a separating covering ring of $G$ being a sub-ring of $\mathcal{R}_{\mu}$ and let $\nu$ be a restriction of $\mu$ on $\mathcal{U}$. Then $\mathcal{U}_{\nu}=\mathcal{R}_{\mu}$ and $\bar{\nu}=\bar{\mu}$.

Proof. Let $u \in \mathcal{S}$ be a semi-norm in $X$. At first we prove that $N_{\mu, u}(x) \geq$ $N_{\nu, u}(x)$ for each $x \in G$. Suppose the contrary, that there exists $y \in G$ such that $N_{\mu, u}(y)<N_{\nu, u}(y)$. This implies that there exists $V \in \mathcal{R}$ such that $\|V\|_{\mu, u}<N_{\nu, u}(y)$.

In accordance with Lemma $50\|V\|_{\mu, u}=\|V\|_{\bar{\mu}, u}$. Then for every $x \in G \backslash V$ there exists $B \in \mathcal{U}$ such that $y \in B$ and $x \notin B$, since $\mathcal{U}$ is the separating covering ring. Therefore, $\{B \backslash V: B \in \mathcal{U}, y \in B\}$ is a shrinking sub-collection of $\mathcal{R}_{\mu}$ whose intersection is empty. In view of $48\left(i i i^{\prime}\right)$ there exists $B \in \mathcal{U}$ such that $y \in B$ and $\|B \backslash V\|_{\bar{\mu}, u}<N_{\nu, u}(y)$. Since $\|V\|_{\bar{\mu}, u}<N_{\mu, u}(y)$. But $\nu$ is the restriction of $\bar{\mu}$ on $\mathcal{U}$ and $B \in \mathcal{U}$, then $\|B\|_{\nu, u} \leq\|B\|_{\bar{\mu}, u}<N_{\nu, u}(y)$ giving the contradiction, since $y \in B$.

Demonstrate now, that $\mathcal{R}_{\mu} \subset \mathcal{U}_{\nu}$. Let $A \in \mathcal{R}_{\mu}$ and $\epsilon>0$. In view of Lemma 47 it is sufficient for each $u \in \mathcal{S}$ to construct $B \in \mathcal{U}$ such that $N_{\nu, u}(x)<\epsilon$ for every $x \in A \triangle B$. Take $W:=\left\{x \in A: N_{\mu, u}(x) \geq \epsilon\right\}$. In view of Theorem 51 the set $W$ is $\mathcal{R}_{\mu}$-compact, consequently, $\mathcal{U}$-compact. Mention that for each $x \in G \backslash W$ there exists $B \in \mathcal{U}$ such that $W \subset B$ with $x \notin B$. Then the shrinking sub-family $\{B \backslash A: B \in \mathcal{U}, B \supset W\}$ of $\mathcal{R}_{\mu}$ has the empty intersection and there exists $B \in \mathcal{U}$ such that $B \supset W$ for which $\|B \backslash A\|_{\bar{\mu}, u}<\epsilon$. Thus $N_{\bar{\mu}, u}=N_{\mu, u}<\epsilon$ on $B \backslash A$. On the other hand, $N_{\mu, u}(x)<\epsilon$ on $A \backslash W$ hence on $A \triangle B$ as well.

Next we show that $\mu$ can be obtained as the restriction of $\bar{\nu}$. For $A, B, \epsilon$, $u$ as in the preceding paragraph we have $u(\bar{\nu}(A)-\bar{\mu}(A))=u(\bar{\nu}(A)-\nu(B)+$ $\bar{\mu}(B)-\bar{\nu}(A))=u(\bar{\nu}(A \backslash(A \cap B))-\bar{\nu}(B \backslash(A \cap B))+\bar{\mu}((B \backslash(A \cap B))-\bar{\mu}(B \backslash$ $(A \cap B))) \leq \max \left(\|A \triangle B\|_{\bar{\nu}, u},\|A \triangle B\|_{\bar{\mu}, u}\right)=\|A \triangle B\|_{\bar{\mu}, u}=\sup _{x \in A \triangle B} N_{\mu, u}(x)$. Therefore, $\bar{\nu}=\bar{\mu}$ on $\mathcal{R}_{\mu}$.

Thus $\mathcal{R} \subset \mathcal{U}_{\nu}$ and $\mu$ is the restriction of $\bar{\nu}$ on $\mathcal{R}$. Symmetrically inter- 
changing $\mu$ and $\nu$ in the proof above one obtains $\mathcal{U}_{\nu}=\mathcal{R}_{\mu}$ and $\bar{\mu}=\bar{\nu}$.

54. Lemma. Let $\mu$ be a $X$-valued measure on $\mathcal{R}$, for a semi-norm $u$ in $X$ and for $\epsilon>0$ put $G_{\epsilon, u}:=\left\{x \in G: N_{\mu, u}(x) \geq \epsilon\right\}$. Then the restriction of the $\mathcal{R}$ and $\mathcal{R}_{\mu}$-topologies to $G_{\epsilon, u}$ coincide. A function $f: G \rightarrow \mathrm{g}$ is $\mathcal{R}_{\mu^{-}}$ continuous if and only if for each $u \in \mathcal{S}$ and $\epsilon>0$ the restriction $\left.f\right|_{G_{\epsilon, u}}$ is $\mathcal{R}$-continuous.

Proof. In view of Lemma 47 the $\mathcal{R}$-topology and $\mathcal{R}_{\mu}$-topology induce the same topology on $G_{\epsilon, u}$. Therefore, if $f: G \rightarrow \mathrm{g}$ is $\mathcal{R}_{\mu}$-continuous, then it is $\mathcal{R}$-continuous on $G_{\epsilon, u}$.

Suppose that $f: G \rightarrow$ g has $\mathcal{R}$-continuous restrictions $\left.f\right|_{G_{\epsilon, u}}$ for each $u \in \mathcal{S}$ and every $\epsilon>0$. Take any $V$ clopen in g. If $A \in \mathcal{R}_{\mu}$, then $A \cap G_{\epsilon, u}$ is $\mathcal{R}$-compact due to Theorem 51, as well as $f^{-1}(V) \cap A \cap G_{\epsilon, u}$ is $\mathcal{R}$-clopen as a subset of $G_{\epsilon, u}$. For each $x \in f^{-1}(V) \cap A \cap G_{\epsilon, u}$ take $U_{x} \in \mathcal{R}$ with $x \in U_{x}$ such that $U_{x} \cap G_{\epsilon, u} \subset f^{-1}(V) \cap A \cap G_{\epsilon, u}$. From this covering of the compact set choose a finite sub-covering such that $f^{-1}(V) \cap A \cap G_{\epsilon, u} \subset U$, where $U:=\bigcup_{j=1}^{k} U_{x_{j}}$, hence $U \in \mathcal{R}$. Therefore, $f^{-1}(V) \cap A \cap G_{\epsilon, u}=U \cap G_{\epsilon, u}$. In view of Lemma $47 f^{-1}(V) \cap A \in \mathcal{R}_{\mu}$ for each $A \in \mathcal{R}$. Thus $f^{-1}(V)$ is $\mathcal{R}_{\mu}$-clopen and inevitably $f$ is $\mathcal{R}_{\mu}$-continuous.

55. Corollary. If $f: G \rightarrow \mathrm{g}$ is $\mathcal{R}_{\mu}$-continuous on each $\mathcal{R}_{\mu}$-compact set, then $f$ is $\mathcal{R}_{\mu}$-continuous on $G$. If $u \in \mathcal{S}$ and $E \subset G$ is $\mathcal{R}_{\mu}$-compact, then $H:=\left\{x \in E: N_{\mu, u}(x)=0\right\}$ is finite and there exists $\delta>0$ such that $N_{\mu, u}>\delta$ on $E \backslash H$.

Proof. In view of Lemma 54 each $\mathcal{R}$-compact set $G_{\epsilon, u}$ is $\mathcal{R}_{\mu}$-compact and $f$ is continuous on every $\mathcal{R}$-compact subset of $G_{\epsilon, u}$. On the other hand, $G_{\epsilon, u}$ is $\mathcal{R}$-locally compact by Corollary 52 . Therefore, $f$ is $\mathcal{R}$-continuous on $G_{\epsilon, u}$ and $\mathcal{R}_{\mu}$-continuous on $G$.

We have that each subset $A$ of $\left\{x \in G: N_{\mu, u}(x)=0\right\}$ is $\mathcal{R}_{\mu}$-clopen, since $C h_{A} \in L(\mu)$. Take $E$ a $\mathcal{R}_{\mu}$-compact subset in $G$. Hence $H$ is finite. Let $\pi \in \mathbf{K}$ with $0<|\pi|<1$. If $\inf \left\{N_{\mu, u}(x): x \in E \backslash H\right\}=0$, then there exists a sequence $\left\{x_{k} \in E: k \in \mathbf{N}\right\}$ in $E$ such that $N_{\mu, u}\left(x_{k}\right)<|\pi|^{k}$ and $N_{\mu, u}\left(x_{k}\right)<N_{\mu, u}\left(x_{k-1}\right)$ for each $k$. Choose $A_{k} \in \mathcal{R}$ such that $x_{k} \in A_{k}$ and $N_{\mu, u}(x)<|\pi|^{k}$ for each $x \in A_{k}$ and $A_{k} \cap A_{l}=\emptyset$ for each $k \neq l$. Without loss of generality we can consider the family of non-archimedean semi-norms $\mathcal{S}$ in $X$ such that if $u, q \in \mathcal{S}$, then $t:=\max (u(x), q(x)) \in \mathcal{S}$ is also a seminorm in $X$ (see also [33]). Therefore, $\|A\|_{\mu, t}=\max \left(\|A\|_{\mu, u},\|A\|_{\mu, q}\right)$ for each $A \in \mathcal{R}$, as well as $N_{\mu, t}(x)=\max \left(N_{\mu, u}(x), N_{\mu, q}(x)\right)$ for each $x \in G$. Hence $G_{\epsilon, q} \cap G_{\epsilon, u} \supset G_{\epsilon, t}$ for each $\epsilon>0$ and $t=\max (u, q), u, q, t \in \mathcal{S}$. If $\left.f\right|_{U}$ is 
continuous on a set $U$ and $W$ is a subset of $U, W \subset U$, then evidently $\left.f\right|_{W}$ is continuous.

In view of Lemma 54 and Theorem 51 the function $g(x):=\sum_{k} \pi^{-k} C h_{A_{k} \cap\left\{x \in G: N_{\mu, u}(x)>0\right\}}(x) v_{k}$

is $\mathcal{R}_{\mu}$-continuous, where $v_{k} \in X, u\left(v_{k}\right)=1$ for each $k$, since the restriction of $g$ on each $G_{\gamma, q}$ is continuous for each $q \in \mathcal{S}$ and $\gamma>0$. But $g$ appears to be not bounded on the $\mathcal{R}_{\mu}$-compact set $E$. This gives the contradiction, consequently, $\inf \left\{N_{\mu, u}(x): x \in E \backslash H\right\}>0$.

56. Theorem. Let $\mu$ be a $X$-valued measure on $\mathcal{R}$. A function $f: G \rightarrow \mathrm{g}$ is $\mu$-integrable if and only if it satisfies (1) and (2):

(1) $f$ is $\mathcal{R}_{\mu}$-continuous;

(2) for each $u \in \mathcal{S}$ and $\epsilon>0$ the $\operatorname{set}\left\{x: x \in G, u(f(x)) N_{\mu, u}(x) \geq \epsilon\right\}$ is $\mathcal{R}_{\mu}$-compact, consequently, contained in some $\left\{x: N_{\mu, u}(x) \geq \delta\right\}$ with $\delta>0$.

Proof. If $u$ is a semi-norm in $X$ and $\epsilon>0$, then the set $G_{\epsilon, u}:=\{x \in$ $\left.G: N_{\mu, u}(x) \geq \epsilon\right\}$ is $\mathcal{R}$-compact by Theorem 51 . Without loss of generality we consider complete $X$ and $\mathrm{g}$. For each $f \in L(G, \mathcal{R}, \mu, X ; \mathrm{g})$ and each seminorm $u$ in $X$ there exists a sequence of simple functions $\left\{f_{k}: k \in \mathbf{N}\right\}$ such that $\lim _{k \rightarrow \infty}\left\|f-f_{k}\right\|_{\mu, u}=0$. Then each $f_{k}$ is $\mathcal{R}$-continuous and the sequence $\left\{f_{k}: k\right\}$ converges uniformly on $G_{\epsilon, u}$ to $f$, hence $f$ is $\mathcal{R}$-continuous on $G_{\epsilon, u}$. In view of Corollary 55 the function $f$ is $\mathcal{R}_{\mu}$-continuous.

Take a step function $g$ such that $\|f-g\|_{\mu, u}<\epsilon$, consequently, $\{x$ : $\left.u(f(x)) N_{\mu, u}(x) \geq \epsilon\right\}=\left\{x: u(g(x)) N_{\mu, u}(x) \geq \epsilon\right\}$ and this set is compact by Theorem 51. Thus from $f \in L(G, \mathcal{R}, \mu, X ; \mathrm{g})$ Properties $(1,2)$ follow.

Let now Properties $(1,2)$ be satisfied for $f: G \rightarrow X$. For $\delta>0$ and a semi-norm $u$ in $X$ take a $\mathcal{R}_{\mu}$-step function $g$ such that $\|f-g\|_{\mu, u}<\delta$. Consider the set $V:=\left\{x \in G: u(f(x)) N_{\mu, u}(x) \geq \delta\right\}$. The function $N_{\mu, u}$ is $\mathcal{R}_{\mu}$-upper semi-continuous by Theorem 51 and $\sup _{x \in C} N_{\mu, u}(x)=: w<\infty$. Since $V$ is compact, then there exists a finite clopen covering $B_{1}, \ldots, B_{k}$ of $V$ such that $u(f(x)-f(y)) w<\delta$ for each $x, y \in B_{j} \cap V$ with the same $j, j=1, \ldots, k$. Choose now $b_{j} \in B_{j}$, hence the set $\{x \in G: u(f(x)-$ $\left.\left.f\left(b_{j}\right)\right) N_{\mu, u}(x)<\delta\right\}$ is $\mathcal{R}_{\mu, u}$-open and contains $B_{j}$. Therefore, there exist disjoint sets $W_{1}, \ldots, W_{k}$ such that $W_{j} \subset\left\{x \in G: u\left(f(x)-f\left(b_{j}\right)\right) N_{\mu, u}(x)<\delta\right\}$ and $B_{j}:=W_{j} \cap V$, since $G$ is $\mathcal{R}$ totally disconnected with the clopen base of its topology. Take the step function $g(x):=\sum_{j=1}^{k} f\left(b_{j}\right) C h_{W_{j}}(x)$. For $x \in W_{j}$ we have $u(f(x)-g(x)) N_{\mu, u}(x)=u\left(f(x)-f\left(b_{j}\right)\right) N_{\mu, u}(x)<\delta$. At the same time for $x \notin \bigcup_{j=1}^{k} W_{j}$ we have $\left.u(f(x)-g(x)) N_{\mu, u}(x)=u(f)\right) N_{\mu, u}(x)<\delta$, consequently, $\|f-g\|_{\mu, u} \leq \delta$. 
57. Corollary. Let $\mu$ be a $X$-valued measure on $\mathcal{R}, g \in L(G, \mathcal{R}, \mu, X ; \mathrm{g})$, $f: G \rightarrow \mathrm{g}$ be $\mathcal{R}_{\mu}$-continuous and $u(f(x)) \leq u(g(x))$ for each semi-norm $u \in \mathrm{g}$ and for every $x \in G$, then $f \in L(G, \mathcal{R}, \mu, X ; \mathrm{g})$.

58. Corollary. The space $L(G, \mathcal{R}, \mu, X ; \mathrm{g})$ is complete and locally $\mathbf{K}$ convex. If $X$ and $\mathrm{g}$ are normed spaces, then $L(G, \mathcal{R}, \mu, X ; \mathrm{g})$ is the Banach space.

Proof. By the construction above $L(G, \mathcal{R}, \mu, X ; \mathrm{g})$ is the completion of the space of step functions relative to the family of semi-norms $\|*\|_{\mu, u}$, where $u$ is a semi-norm in $X$, g. Therefore, $L(G, \mathcal{R}, \mu, X ; \mathrm{g})$ is isomorphic with $L(G, \mathcal{R}, \mu, \tilde{X} ; \mathrm{g})$ and complete, where $\tilde{X}$ is the completion of $X$ and $\tilde{\mathrm{g}}$ is the completion of $\mathrm{g}$ as the $\mathbf{K}$-convex spaces. Particularly, when $X$ and $\mathbf{g}$ are normed spaces, then $\tilde{X}$ and $\tilde{\mathrm{g}}$ and $L(G, \mathcal{R}, \mu, \tilde{X} ; \tilde{\mathrm{g}})$ are the Banach spaces.

59. Definition. If $X$ is a normed space over $\mathbf{K}$ and $x_{1}, x_{2}, \ldots$ is a sequence in $X$ such that $\left\|a_{1} x_{1}+\ldots+a_{n} x_{n}\right\| \geq t \max \left\{\left\|a_{j} x_{j}\right\|: j=1, \ldots, n\right\}$ for each $a_{1}, \ldots, a_{n} \in \mathbf{K}$ and $n \in \mathbf{N}$ not exceeding the length of the sequence, where $0<t \leq 1$ is a marked number, then $\left\{x_{1}, x_{2}, \ldots\right\}$ are called $t$-orthogonal. If $t=1$, then $\left\{x_{1}, x_{2}, \ldots\right\}$ are called orthogonal.

Naturally in the case $t=1$ the inequality, $\geq$, reduces to the equality, $=$, due to the non-archimedean property of the norm.

60. Theorem. Let $\mu$ be a $X$-valued measure on $(G, \mathcal{R})$ and let a Banach algebra $\mathrm{g}$ has a $t_{0}$-orthogonal basis, where $0<t_{0} \leq 1$. Then $L(G, \mathcal{R}, \mu, X ; \mathrm{g})$ has a t-orthogonal basis with $0<t<t_{0}$. If the valuation group of the field $\mathbf{K}$ is discrete in $(0, \infty)$, then $L(G, \mathcal{R}, \mu, X ; \mathbf{g})$ has an orthogonal basis.

Proof. If the valuation group of $\mathbf{K}$ is discrete, then the Banach space over $\mathbf{K}$ has an orthogonal basis by Theorem 5.16 [37], particularly, for $L(G, \mathcal{R}, \mu, X ; \mathrm{g})$ due to Corollary 58.

In general, suppose that the valuation group of $\mathbf{K}$ is dense in $(0, \infty)$ and $N_{\mu}(x)>0$ for each $x \in G$. Take a marked $0<t<t_{0}$ such that $\mathrm{g}$ has the $t_{0}$-orthogonal basis. Then choose $\pi \in \mathbf{K}$ such that $t_{1}<|\pi|<1$, where $t_{1}=t / t_{0}$, and define the function $h: G \rightarrow \mathbf{K}$ such that $h(x)=\pi^{n}$, when $|\pi|^{n+1}<N_{\mu}(x) \leq|\pi|^{n}$ and $n \in \mathbf{Z}$, consequently, $|\pi||h(x)|<N_{\mu}(x) \leq|h(x)|$ for each $x \in G$. Denote by $G_{d}$ the set $G$ in the discrete topology and define the mapping $q: L(G, \mathcal{R}, \mu, X ; \mathrm{g}) \ni f \mapsto h f \in B C\left(G_{d}, \mathrm{~g}\right)$, where $B C(Y, W)$ denotes the space of all bounded continuous mappings from a topological space $Y$ into a $\mathbf{K}$-linear normed space $W$. The space $B C\left(G_{d}, \mathbf{g}\right)$ is supplied with the norm $\|v\|_{\infty}:=\sup _{x \in G_{d}}\|v(x)\|$, where $\|*\|$ is the norm in $\mathrm{g}$. Therefore, 
(1) $|\pi|\|q f\|_{\infty}<\|f\|_{\mu} \leq\|q f\|_{\infty}$

for each $f \in L(G, \mathcal{R}, \mu, X ; \mathrm{g})$. If $A \in \mathcal{R}$ and $b \in \mathrm{g}$, then $h b C h_{A} \in B C\left(G_{d}, \mathrm{~g}\right)$, since $\left.\| h(x) b C h_{A}(x): x \in G\right\} \subset\left\{\pi^{n}: n \in \mathbf{Z} ;|\pi|^{n+1}<\|b\|\left|C h_{A}(x)\right|\right\}$.

By $\mathbf{K}$ linearity and Property (1) of the mapping $q$ the range $q(L(G, \mathcal{R}, \mu, X ; \mathbf{g}))$

is the closed $\mathbf{K}$-linear subspace in $B C\left(G_{d}, \mathbf{g}\right)$. In the space $B C\left(G_{d}, \mathbf{g}\right)$ the product $B C\left(G_{d}, \mathbf{K}\right) \times \mathrm{g}$ is everywhere dense, while $L(G, \mathcal{R}, \mu, X ; \mathbf{K}) \times \mathrm{g}$ is everywhere dense in $L(G, \mathcal{R}, \mu, X ; \mathrm{g})$. In view of Corollaries 5.23 and 5.25 [37] $B C\left(G_{d}, \mathbf{K}\right)$ has an orthogonal basis, hence $B C\left(G_{d}, \mathbf{g}\right)$ has a $t_{0}$-orthogonal basis.

In accordance with the Gruson Theorem 5.9 [37] if $E$ is a Banach space with an orthogonal basis, then each its closed $\mathbf{K}$-linear subspace has an orthogonal basis. The space $q(L(G, \mathcal{R}, \mu, X ; \mathbf{K}))$ is closed in $B C\left(G_{d}, \mathbf{K}\right)$, hence it has an orthogonal basis $\left\{e_{j}: j\right\}$. Thus $L(G, \mathcal{R}, \mu, X ; \mathrm{g})$ has the $t$-orthogonal basis $q^{-1}\left(e_{j} \times s_{k}\right)$, where $\left\{s_{k}: k\right\}$ is the $t_{0}$-orthogonal basis in $\mathrm{g}$.

61. Theorems. Let $X$ and $\mathrm{g}$ be as in $\S 39$ and in addition let $X$ be an algebra over $\mathbf{K}$ with a family of multiplicative semi-norms. Suppose that $\mu$ and $\nu$ are $X$-valued measures on separating covering rings $\mathcal{R}$ of a set $G$ and $\mathcal{T}$ of a set $H$. Then

(1) the finite unions of the sets $A \times B, A \in \mathcal{R}, B \in \mathcal{T}$ form the separating covering ring $\mathcal{R} \otimes \mathcal{T}$ of $G \times H$;

(2) there exists a unique measure $\mu \times \nu$ on $\mathcal{R} \times \mathcal{T}$ such that $\mu \times \nu(A \times B)=$ $\mu(A) \nu(B)$ for each $A \in \mathcal{R}$ and $B \in \mathcal{T}, N_{\mu \times \nu, u}(x, y)=N_{\mu, u}(x) N_{\nu, u}(y)$ for each $x \in G, y \in H$ and every semi-norm $u \in \mathcal{S}$ in $X$;

(3) if $f \in L\left(G \times H, \mathcal{R} \times \mathcal{T}, \mu \times \nu, X\right.$;g), then $H \ni y \mapsto \int_{G} f(x, y) \mu(d x)$ is a $\nu$-almost everywhere defined $\nu$-integrable function on $H$ and $G \ni x \mapsto$ $\int_{H} f(x, y) \nu(d y)$ is a $\mu$-almost everywhere defined $\mu$-integrable function on $G$ and

$\int_{G \times H} f(x, y) \mu \times \nu(d x, d y)=\int_{H}\left(\int_{G} f(x, y) \mu(d x)\right) \nu(d y)$. Moreover, if $X$ is commutative, then $\int_{H}\left(\int_{G} f(x, y) \mu(d x)\right) \nu(d y)=\int_{G}\left(\int_{H} f(x, y) \nu(d x)\right) \mu(d y)$;

(4) in particular, if $\mathrm{g}$ and $X$ are commutative, $f \in L(G, \mathcal{R}, \mu, X ; \mathrm{g})$ and $h \in L(H, \mathcal{T}, \nu, X ; \mathrm{g})$, then $f(x) h(y) \in L(G \times H, \mathcal{R} \times \mathcal{T}, \mu \times \nu, X ; \mathbf{g})$ and

$\int_{G \times H} f(x) g(y) \mu(d x) \nu(d y)=\left(\int_{G} f(x) \mu(d x)\right)\left(\int_{H} g(y) \nu(d y)\right) ;$

(5) $L(G \times H, \mathcal{R} \times \mathcal{T}, \mu \times \nu, X ; \mathbf{g})$ is $\mathbf{K}$-linearly topologically isomorphic with the tensor product $L(G, \mathcal{R}, \mu, X ; \mathrm{g}) \hat{\otimes} L(H, \mathcal{T}, \nu, X ; \mathrm{g})$.

Proof. (1). If $\mathcal{U}$ and $\mathcal{V}$ are coverings of $G$ and $H$ by elements from $\mathcal{R}$ and $\mathcal{T}$ respectively, then $\bigcup_{A \in \mathcal{U}, B \in \mathcal{V}} A \times B=G \times H$. If $\left(x_{1}, y_{1}\right) \neq\left(x_{2}, y_{2}\right) \in G \times H$, then either $x_{1} \neq x_{2}$ or $y_{1} \neq y_{2}$. In the first case take $A \in \mathcal{R}$ such that $x_{1} \in A$ 
and $x_{2} \in G \backslash A$ and $x_{1} \in B$ with $B \in \mathcal{T}$, then $A \times B$ separates them: $\left(x_{1}, y_{1}\right) \in A \times B$ and $\left(x_{2}, y_{2}\right) \notin A \times B$.

(2). Put $\mu \times \nu(E):=\int_{G \times H} C h_{C}(x, y) \mu(d x) \nu(d y)$ for each $E \in \mathcal{R} \times \mathcal{T}$, hence $\mu \times \nu$ is additive. For $N_{u}(x, y):=N_{\mu, u}(x) N_{\nu, u}(y)$ and each $A \in \mathcal{R}$, $B \in \mathcal{T}$ we get $u((\mu \times \nu))(A \times B)) \leq\left\|C h_{A \times B}\right\|_{N_{u}}$, consequently, $\|C\|_{\mu \times \nu, u} \leq$ $\left\|C h_{C}\right\|_{N_{u}}$.

Naturally $G$ and $H$ and $G \times H$ are supplied with the $\mathcal{R}$ and $\mathcal{T}$ and $\mathcal{R} \times \mathcal{T}$ topologies. Then $\left\{(x, y) \in A \times B: N_{u}(x, y) \geq \epsilon\right\} \subset\left\{x \in A: N_{\mu, u}(x) \geq\right.$ $\epsilon\} \times\left\{y \in B: N_{\nu, u}(y) \geq \epsilon\right\}$ for each $A \in \mathcal{R}$ and $B \in \mathcal{T}$ and $\epsilon>0$. The function $N_{u}$ is upper semi-continuous and $\left\{x \in A: N_{\mu, u}(x) \geq \epsilon\right\} \times\{y \in$ $\left.B: N_{\nu, u}(y) \geq \epsilon\right\}$ is compact, consequently, $\left\{(x, y) \in A \times B: N_{u}(x, y) \geq \epsilon\right\}$ is compact and inevitably $\left\{(x, y) \in C: N_{u}(x, y) \geq \epsilon\right\}$ is compact for each $C \in \mathcal{R} \times \mathcal{T}$ and every $\epsilon>0$. Therefore, by Theorem $51 \mu \times \nu$ is the measure and $N_{\mu \times \nu, u}(x, y) \leq N_{u}(x, y)$ for each $u \in \mathcal{S}$ and each $x \in G$ and every $y \in H$. Each $u$ is the multiplicative semi-norm in $X$ and $u((\mu \times \nu)(A \times B))=$ $u(\mu(A)) u(\nu(B))$ and hence $\sup \{u((\mu \times \nu)(C)): C \in \mathcal{R} \times \mathcal{T}, C \subset A \times B\}=$ $\|A \times B\|_{\mu \times \nu, u} \geq\|A\|_{\mu, u}\|B\|_{\nu, u}$ for each $A \in \mathcal{R}$ and every $B \in \mathcal{T}$. Therefore, $N_{\mu \times \nu, u}(x, y) \geq N_{u}(x, y)$ for each $u, x$ and $y$.

(3). If $f$ is a step function, then (3) is satisfied due to (2). If $f \in$ $L(G, \mathcal{R}, \mu, X ; \mathrm{g})$, then for each $u$ there exists a sequence of step functions $f_{1}, f_{2}, \ldots$ converging to $f$ such that $\left\|f-f_{n}\right\|_{N_{u}} \leq 1 / n$ for each $n \in \mathbf{N}$. Since $u\left(f(x, y)-f_{n}(x, y)\right) N_{\mu, u}(x) N_{\nu, u}(y) \leq 1 / n$ for each $x, y$. Thus $f(*, y) \in$ $L(G, \mathcal{R}, \mu, X ; \mathrm{g})$ for $\nu$-almost every $y \in H$, consequently, $u\left(\int_{G} f(x, y) \mu(d x)-\right.$ $\left.\int_{G} f_{n}(x, y) \mu(d x)\right) N_{\nu, u}(y) \leq 1 / n$. Therefore, the function $H \ni y \mapsto \int_{G} f(x, y) \mu(d x)$ is defined for $\nu$-almost all $y \in H$ and is $\nu$-integrable. Since

$\int_{H}\left(\int_{G} f_{n}(x, y) \mu(d x)\right) \nu(d y)=\int_{G \times H} f_{n}(x, y) \mu \times \nu(d x, d y)$ for each $n$, then $u\left(\int_{H}\left(\int_{G} f_{n}(x, y) \mu(d x)\right) \nu(d y)-\int_{G \times H} f_{n}(x, y) \mu \times \nu(d x, d y)\right) \leq 1 / n$ for each $n \in \mathbf{N}$ and each $u \in \mathcal{S}$, consequently,

$\int_{H}\left(\int_{G} f(x, y) \mu(d x)\right) \nu(d y)=\int_{G \times H} f(x, y) \mu \times \nu(d x, d y)$.

Part (4) follows from $(2,3)$ as the particular case.

(5). There is a bilinear continuous mapping $\mathrm{m}: L(G, \mathcal{R}, \mu, X ; \mathrm{g}) \times$ $L(H, \mathcal{T}, \nu, X ; \mathrm{g}) \ni(f, h) \mapsto f h \in L(G \times H, \mathcal{R} \times \mathcal{T}, \mu \times \nu, X ; \mathrm{g})$. If $X$ is a Banach space, then the norm of $\mathrm{m}$ is $\|\mathrm{m}\|=1$.

Let $Y$ be a complete locally $\mathbf{K}$-convex space over $\mathbf{K}$ and let $F: L(G, \mathcal{R}, \mu, X ; \mathbf{g}) \times$ $L(H, \mathcal{T}, \nu, X ; \mathbf{g}) \rightarrow Y$ be a continuous $\mathbf{K}$-bilinear mapping. Then there exists the mapping $F_{\mathrm{m}}\left(C h_{A \times B}\right)=F_{\mathrm{m}}\left(C h_{A} \otimes C h_{B}\right)=F\left(C h_{A}, C h_{B}\right) \in Y$ for each $A \in \mathcal{R}$ and $B \in \mathcal{T}$. A step function $f: G \times H \rightarrow \mathrm{g}$ write in the form 
$f(x, y)=\sum_{j} b_{j} C h_{A_{j} \times B_{j}}(x, y)$, where $b_{j} \in \mathrm{g}$ and $A_{j} \in \mathcal{R}, B_{j} \in \mathcal{T}$ for each $j$, $\left(A_{j} \times B_{j}\right) \cap\left(A_{i} \times B_{i}\right)=\emptyset$ for each $i \neq j$.

For each semi-norm $v$ in $Y$ and each semi-norm $u$ in $X$ there exists $k$ such that

$\sup _{x \in G, y \in H} v\left(F_{\mathrm{m}}\left(b_{k} C h_{A_{k} \times B_{k}}\right)(x, y)\right) N_{\mu \times \nu, u}(x, y)$

$=\max _{j} v\left(F_{\mathrm{m}}\left(b_{j} C h_{A_{j} \times B_{j}}\right)(x, y)\right) N_{\mu \times \nu, u}(x, y)$, hence

$v\left(F_{\mathrm{m}} f(x, y)\right) N_{\mu \times \nu, u}(x, y) \leq \sup _{x \in G, y \in H} v\left(F_{\mathrm{m}}\left(b_{k} C h_{A_{k} \times B_{k}}\right)(x, y)\right) N_{\mu \times \nu, u}(x, y) \leq$ $u\left(b_{j}\right)\|F\|_{u, v}\left\|C h_{A_{k}}\right\|_{\mu, u}\left\|C h_{B_{k}}\right\|_{\nu, u} \leq\|F\|_{u, v}\|f\|_{\mu \times \nu, u}$,

where $\|F\|_{u, v}:=\sup _{w \in X, u(w)>0} v(F w) / u(w)$.

Therefore, the mapping $F_{\mathrm{m}}$ has the continuous extension $F_{\mathrm{m}}$ from $L(G \times$ $H, \mathcal{R} \times \mathcal{T}, \mu \times \nu, X ; \mathrm{g})$ into $Y$. Thus $F_{\mathrm{m}}(f \otimes h)=F(f, h)$ for each $f \in$ $L(G, \mathcal{R}, \mu, X ; \mathrm{g})$ and $h \in L(H, \mathcal{T}, \nu, X ; \mathrm{g})$ and inevitably $L(G \times H, \mathcal{R} \times$ $\mathcal{T}, \mu \times \nu, X ; \mathrm{g})$ is $\mathbf{K}$-linearly topologically isomorphic with the tensor product $L(G, \mathcal{R}, \mu, X ; \mathrm{g}) \hat{\otimes} L(H, \mathcal{T}, \nu, X ; \mathrm{g})$.

62. Theorem. Let $\mathrm{g}_{j}$ and $X_{j}$ be a family of algebras and locally convex spaces over $\mathbf{K}$ satisfying Conditions of $\S 39, j \in \beta$, where $\beta$ is a set. Suppose that for each $j$ there is a measure $\mu_{j}: \mathcal{R} \rightarrow X_{j}, X=\bigotimes_{j \in \beta} X_{j}$ and $\mathrm{g}=\bigotimes_{j \in \beta} \mathrm{g}_{j}$ are supplied with the product topologies. Then there exists a measure $\mu=\bigotimes_{j \in \beta} \mu_{j}$ on $\mathcal{R}$ with values in $X$ such that $L(G, \mathcal{R}, \mu, X ; \mathrm{g})$ is the completion of the direct sum $\bigoplus_{j \in \beta} L\left(G, \mathcal{R}, \mu_{j}, X_{j} ; \mathrm{g}_{j}\right)$.

Proof. Since each $X_{j}$ and every $\mathrm{g}_{j}$ are complete, then $X$ and $\mathrm{g}$ are complete (see $[10,33])$. Naturally $X$ is the locally $\mathbf{K}$-convex space and $\mathbf{g}$ is the algebra such that $x+y=\left(x_{j}+y_{j}: j\right)$ and $a b=\left(a_{j} b_{j}: j\right)$, where $x, y \in X, a, b \in \mathrm{g}, x=\left(x_{j}: j\right), a=\left(a_{j}: j\right), a x=\left(a_{j} x_{j}: j\right)$ (see Theorem 5.6.1 [33]). Thus $X$ is the unital left g-module. For each $j \in \beta$ there are defined the projectors $\pi_{j}(x)=x_{j}$ and $\pi_{j}(a)=a_{j}$ on $X$ and $\mathrm{g}$. Therefore, topologies of $X$ and $\mathrm{g}$ are characterized by the families of semi-norms $u$ such that $u(x)=\max \left\{u_{j}\left(x_{j}\right): j \in \alpha\right\}$ and $u(b)=\max \left\{u_{j}\left(b_{j}\right): j \in \alpha\right\}$, where $\alpha$ is a finite subset in $\beta, u_{j} \in \mathcal{S}_{j}$, where $\mathcal{S}_{j}$ is a consistent family of semi-norms $u_{j}$ in $X_{j}$ and $\mathrm{g}_{j}$ denoted by the same symbol for shortening the notation.

If $A, B \in \mathcal{R}$ and $A \cap B=\emptyset$, then $\mu(A \cup B)=\left(\mu_{j}(A \cup B): j\right)=$ $\left(\mu_{j}(A)+\mu_{j}(B): j\right)=\left(\mu_{j}(A): j\right)+\left(\mu_{j}(B): j\right)=\mu(A)+\mu(B)$, hence $\mu$ is additive. If $u$ is a semi-norm in $X$ and $A \in \mathcal{R}$, then for each $C \subset A, C \in \mathcal{R}$ there is the inequality $u(\mu(C))=\max \left\{u_{j}\left(\mu_{j}(C)\right): j \in \alpha\right\}<\infty$, since $\alpha$ is a finite set and each $\mu_{j}$ is bounded. If $\mathcal{A}$ is a shrinking family in $\mathcal{R}$ and $\bigcap_{A \in \mathcal{A}} A=\emptyset$, then $\lim _{A \in \mathcal{A}} u_{j}\left(\mu_{j}(A)\right)=0$ for each $j$ and each $u_{j} \in \mathcal{S}_{j}$, hence $\lim _{A \in \mathcal{A}} u(\mu(A))=0$ for each semi-norm $u \in \mathcal{S}$ in $X$. Thus $\mu$ is the measure 
on $\mathcal{R}$ with values in $X$.

If $f \in L(G, \mathcal{R}, \mu, X ; \mathrm{g})$, then for each $u \in \mathcal{S}$ there exists a sequence $\left\{f_{n}: n \in \mathbf{N}\right\}$ of simple functions such that $\left\|f-f_{n}\right\|_{\mu, u} \leq 1 / n$. We have that $\bigoplus_{j} \mathrm{~g}_{j}$ is everywhere dense in $\mathrm{g}$, where elements of the direct sum as usually are $b=\left(b_{j}: j \in \beta, b_{j} \in \mathrm{g}_{j}\right\}$ such that the set $\left\{j: b_{j} \neq 0\right\}$ is finite (see Example 5.10.6 in [33]). Thus each simple function can be chosen taking values in $\bigoplus_{j \in \beta} \mathrm{g}_{\mathbf{j}}$. Therefore, $L(G, \mathcal{R}, \mu, X ; \mathrm{g})$ is the completion of the direct $\operatorname{sum} \bigoplus_{j \in \beta} L\left(G, \mathcal{R}, \mu_{j}, X_{j} ; \mathrm{g}_{j}\right)$.

Certainly if $\beta$ is finite, then the direct sum and the direct product coincide.

63. Corollary. If suppositions of Theorem 62 are satisfied and $f \in$ $L(G, \mathcal{R}, \mu, X ; \mathrm{g})$, then $\int_{G} f(x) \mu(d x)=\left(\int_{G} f_{j}(x) \mu_{j}\left(d x_{j}\right): j \in \beta\right)$, where $f_{j} \in$ $L\left(G, \mathcal{R}, \mu, X_{j} ; \mathrm{g}_{j}\right)$ for each $j$.

Proof. The formula of this Corollary is satisfied for each $f$ in $\bigoplus_{j \in \beta} L\left(G, \mathcal{R}, \mu_{j}, X_{j} ; \mathrm{g}_{j}\right)$, but the latter space is everywhere dense in $L(G, \mathcal{R}, \mu, X ; \mathrm{g})$ by Theorem 61 . The mapping $\int_{G}: L(G, \mathcal{R}, \mu, X ; \mathrm{g}) \rightarrow X$ is continuous: $u\left(\int_{G} f(x) \mu(d x)\right) \leq\|f\|_{\mu, u}$ due to Lemma 45 , consequently, the statement of this Corollary follows by the continuity.

64. Theorem. Let $\mathrm{g}, X, G, \mathcal{R}, \mu$ be as in $\S 39$ and let $F$ be a continuous homomorphism of a left unital $\mathrm{g}$-module $X$ into a uniformly complete left unital h-module $Y$. Then $F$ induces a continuous homomorphism $\hat{F}$ : $L(G, \mathcal{R}, \mu, X ; \mathrm{g}) \rightarrow L(G, \mathcal{R}, \nu, Y ; \mathrm{h})$ such that $F\left(\int_{G} f(x) \mu(d x)\right)=\int_{G} \hat{F}(f) \nu(d x)$ for each $f \in L(G, \mathcal{R}, \mu, X ; \mathrm{g})$, where $\nu=F(\mu)$ is an $Y$-valued measure. If $F(\mathrm{~g})=\mathrm{h}$, then $\hat{F}$ is epimorphic.

Proof. If $A, B \in \mathcal{R}$ with $A \cap B=\emptyset$, then $\nu(A \cup B):=F(\mu(A \cup B))=$ $F(\mu(A))+F(\mu(B))=\nu(A)+\nu(B) \in Y$. If $A \in \mathcal{R}$ and $u$ is a semi-norm in $X$, then $\sup _{C \subset A, C \in \mathcal{R}} u(\mu(C))<\infty$, hence for each semi-norm $v$ in $Y$ there is the inequality $\sup _{C \subset A, C \in \mathcal{R}} v(\nu(C))<\infty$, since $F$ is continuous. If $\mathcal{A}$ is a shrinking family in $\mathcal{R}$ with $\bigcap_{A \in \mathcal{A}} A=\emptyset$, then $\lim _{\mathcal{A}} \mu(A)=0$ hence $0=\lim _{A \in \mathcal{A}} F(\mu(A))=\lim _{A \in \mathcal{A}} \nu(A)$, since $F$ is continuous. Thus $\nu$ is the $Y$-valued measure.

If $v$ is a semi-norm in $Y$, then $v_{F}(q):=v(F(q))$ is the continuous seminorm in $X$, where $q \in X$, hence $v_{F}(\mu(A))=v(\nu(A))$ for each $A \in \mathcal{R}$ and inevitably $N_{\mu, v_{F}}(x)=N_{\nu, v}(x)$ for each $x \in G$.

If $f: G \rightarrow \mathrm{g}$ is a step function $f(x)=\sum_{j} a_{j} C h_{A_{j}}(x)$, where $a_{j} \in \mathrm{g}$, $A_{j} \in \mathcal{R}$, then $\hat{F}(f)=F(f)=\sum_{j} F\left(a_{j}\right) C h_{A_{j}}(x)$, since $F\left(a_{1} b_{1}+a_{2} b_{2}\right)=$ $F\left(a_{1}\right) F\left(b_{1}\right)+F\left(a_{2}\right) F\left(b_{2}\right)$ for each $a_{j}, b_{j} \in \mathrm{g}$, also $0,1 \in \mathrm{g}, F(0)=0, F(1)=$ 
$1 \in \mathrm{h}$. Moreover, $\|F(f)\|_{\nu, v}=\|f\|_{\mu, v_{F}}$ for each step function $f$ and each semi-norm $v$ in $Y$, consequently, $\hat{F}$ is continuous and $\mathbf{K}$-linear and has the continuous extension $\hat{F}: L(G, \mathcal{R}, \mu, X ; \mathrm{g}) \rightarrow L(G, \mathcal{R}, \nu, Y ; \mathrm{h})$.

For each $s \in L(G, \mathcal{R}, \nu, Y ; \mathrm{h})$ and each semi-norm $v$ in $Y$ take a sequence $s_{n}$ of simple functions $s_{n}: G \rightarrow$ h converging to $s$ such that $\left\|s-s_{n}\right\|_{\nu, v} \leq 1 / n$ for each $n$. If $F(\mathrm{~g})=\mathrm{h}$, then for each $s_{n}=\sum_{j} b_{j, n} C h_{A_{j}}$ there exists a simple function $f_{n}=\sum_{j} a_{j, n} C h_{A_{j}}$ such that $f_{n}: G \rightarrow \mathrm{g}$ and $\hat{F}\left(f_{n}\right)=s_{n}, a_{j, n} \in \mathrm{g}$ for each $j, n$, where $b_{j, n} \in \mathrm{h}, A_{j} \in \mathcal{R}$. But $\left\{f_{n}: n\right\}$ is a fundamental sequence relative to $\|*\|_{\mu, v_{f}}$, consequently, there exists $f \in L(G, \mathcal{R}, \mu, X ; \mathrm{g})$ such that $\hat{F}(f)=s$.

By the conditions of this theorem $F\left(a_{1} w_{1}+a_{2} w_{2}\right)=F\left(a_{1}\right) F\left(w_{1}\right)+$ $F\left(a_{2}\right) F\left(w_{2}\right)$ for each $a_{1}, a_{2} \in \mathrm{g}, w_{1}, w_{2} \in X$, where $F\left(a_{j}\right) \in \mathrm{h}$ and $F\left(w_{j}\right) \in Y$. Therefore, for each step function $f(x)=\sum_{j} a_{j} C h_{A_{j}}(x)$ we have $F\left(\int_{G} f(x) \mu(d x)\right)=$ $F\left(\sum_{j} a_{j} \mu\left(A_{j}\right)\right)=\sum_{j} F\left(a_{j}\right) \nu\left(A_{j}\right)=\int_{G} \hat{F}(f)(x) \nu(d x)$ and $v_{F}\left(\int_{G} f(x) \mu(d x)\right)=$ $v\left(\int_{G} \hat{F}(f)(x) \nu(d x)\right)$ for each semi-norm $v$ in $Y$, consequently, by the continuity $F\left(\int_{G} f(x) \mu(d x)\right)=\int_{G} \hat{F}(f)(x) \nu(d x)$ for each $f \in L(G, \mathcal{R}, \mu, X ; \mathrm{g})$.

65. Definition. Let $\mathcal{R}=\operatorname{Bco}(G)$ be the ring of all clopen subsets of a zero-dimensional Hausdorff space. A measure $\mu: \operatorname{Bco}(G) \rightarrow X$ is called a tight measure, where $X$ is as in $\S 39$. The family $M=M(G, X)$ of all such tight measures form the $\mathbf{K}$-linear space with the family of semi-norms

$$
\|\mu\|_{u}=\sup _{A \in \operatorname{Bco}(G)} u(\mu(A))=\|G\|_{\mu, u}=\sup _{x \in G} N_{\mu, u}(x),
$$

where $u \in \mathcal{S}$ is a semi-norm in $X$. In particular, if $X$ is the normed space, then $M(G, X)$ is the normed space.

The closure of the set $\left\{x \in G: \exists u \in \mathcal{S}, N_{\mu, u}(x)>0\right\}$ we call the support of the measure $\mu$.

66. Theorem. If $\mathcal{R}$ is a covering ring of $G$ being the base of the zero-dimensional Hausdorff topology in $G$ and if $\mu$ is a $X$-valued measure on $\mathcal{R}$, then $(f \mu)(A):=\int_{G} C h_{A}(x) f(x) \mu(d x)$ is the tight measure for $f \in$ $L(G, \mathcal{R}, \mu, X ; \mathbf{K})$ and the mapping $\psi_{\mu}:=\psi: L(G, \mathcal{R}, \mu, X ; \mathbf{K}) \ni \mapsto(f \mu)$ is the $\mathbf{K}$-linear topological embedding $L(G, \mathcal{R}, \mu, X ; \mathbf{K})$ into $M(G, X)$.

Proof. For each $u \in \mathcal{S}$ the set $\left\{x \in G: N_{\mu, u}(x)>0\right\}$ is $\sigma$-compact, that is the countable union of compact subsets by Theorem 51. Therefore, if $A$ is a clopen subset in $G$, then $C h_{A} \in L(G, \mathcal{R}, \mu, X ; \mathbf{K})$, since for each $u \in \mathcal{S}$ and $\epsilon>0$ there exists a sequence $f_{n} \in L(G, \mathcal{R}, \mu, X ; \mathbf{K})$ with $\left\|C h_{A}-f_{n}\right\|_{\mu, u} \leq 1 / n$ and supports $\operatorname{supp}\left(f_{n}\right) \supset\left\{x \in G: N_{\mu, u}(x) \geq 1 / n\right\}$. Thus $f \mu$ is defined on $\mathcal{R}_{f \mu} \supset \operatorname{Bco}(G)$, consequently, $f \mu \in M(G, X)$. Evidently $\psi(a f+b g)=$ 
$a \psi(f)+b \psi(g)=a f \mu+b g \mu$. On the other hand, $N_{f \mu, u}(x) \leq\|f\|_{\mu, u} N_{\mu, u}(x)$, hence $\psi$ is continuous. In view of Theorem $56 u(f(x)) N_{\mu, u}(x)=N_{f \mu, u}(x)$ for each $u \in \mathcal{S}$ and $x \in G$, hence $\psi$ is the topological embedding. If $X$ is a Banach space, then $\psi$ is the isometric embedding.

67. Let $Y^{*}$ denote the topological dual space of all continuous $\mathbf{K}$-linear functionals on a $\mathbf{K}$-linear space $Y, \operatorname{Mat}_{n}(\mathbf{K})$ denotes the algebra of all square $n \times n$ matrices, $n \in \mathbf{N}, B C(G, Y)$ denotes the space of all continuous bounded functions from $G$ into $Y$.

Theorem. If $G$ is a zero-dimensional Hausdorff space and $\mu \in M\left(G, \operatorname{Mat}_{n}(\mathbf{K})\right)$, then $B C\left(G, \operatorname{Mat}_{n}(\mathbf{K})\right) \subset L\left(G, \mathcal{R}, \mu, \operatorname{Mat}_{n}(\mathbf{K}) ; \operatorname{Mat}_{n}(\mathbf{K})\right)$ and $\lambda_{\mu}(f):=\int_{G} f(x) \mu(d x)$ provides the $\mathbf{K}$-linear isometric embedding $\lambda: M\left(G, \operatorname{Mat}_{n}(\mathbf{K})\right) \hookrightarrow B C\left(G, \operatorname{Mat}_{n}(\mathbf{K})\right)^{*}$. If $G$ is compact, then $\lambda$ is the isomorphism.

Proof. In view of Theorem 56 we get the inclusion $B C\left(G, \operatorname{Mat}_{n}(\mathbf{K})\right) \subset$ $L\left(G, \mathcal{R}, \mu, \operatorname{Mat}_{n}(\mathbf{K}) ; \operatorname{Mat}_{n}(\mathbf{K})\right)$. Moreover, the mapping $\lambda$ is defined and it is $\mathbf{K}$-linear.

Since $\operatorname{Mat}_{n}(\mathbf{K})$ is finite dimensional over $\mathbf{K}$, then its topological dual space is isomorphic with $\operatorname{Mat}_{n}(\mathbf{K})$. It has the natural norm topology $\|b\|:=$ $\max _{1 \leq i, j \leq n}\left|b_{i, j}\right|$. If $\mu \in M\left(G, \operatorname{Mat}_{n}(\mathbf{K})\right)$, then $\|\mu\|=\sup _{A \in \operatorname{Bco}(G)} \max _{i, j}\left|\mu_{i, j}(A)\right|=$ $\sup _{A \in \mathrm{Bco}(G)}\left|\lambda_{\mu}\left(C h_{A}\right)\right| \leq\left\|\lambda_{\mu}\right\|$, consequently, $\lambda$ is the isometric embedding.

If $q \in B C\left(G, \operatorname{Mat}_{n}(\mathbf{K})\right)^{*}$, then $\mu(A)$ having matrix elements $q\left(E_{i, j} C h_{A}\right)=$ : $\mu_{i, j}^{q}(A)$ is and additive function on $\operatorname{Bco}(G)$ with values in $\operatorname{Mat}_{n}(\mathbf{K})$, where $E_{i, j}$ is the $n \times n$ matrix with 1 at the $(i, j)$-th place and zeros at others places. For each $A \in \operatorname{Bco}(G)$ and $b \in \operatorname{Mat}_{n}(\mathbf{K})$ we have $b C h_{A} \in B C\left(G, \operatorname{Mat}_{n}(\mathbf{K})\right)$. Therefore, $\mu^{q}$ is defined on $\operatorname{Bco}(G)$. If $\mathcal{A} \subset \operatorname{Bco}(G)$ is a shrinking family and $G$ is compact, then from $\bigcap_{A \in \mathcal{A}} A=\emptyset$ it follows, that $\emptyset \in \mathcal{A}$, since each $A \in \mathcal{A}$ is closed in $G$. Since $q$ is continuous, then for compact $G$ the mapping $\mu^{q}$ is the measure. In this particular case $B C\left(G, \operatorname{Mat}_{n}(\mathbf{K})\right)$ is isomorphic with the space $C\left(G, \operatorname{Mat}_{n}(\mathbf{K})\right)$ of all continuous functions from $G$ into $\operatorname{Mat}_{n}(\mathbf{K})$.

68. Theorem. A function $f: G \rightarrow \mathrm{g}$ is $\mu$-integrable for each $\mu \in$ $M(G, X)$ if and only if $f$ is bounded and for each compact subset $V$ in $G$ the restriction $\left.f\right|_{V}$ of $f$ to $V$ is continuous.

Proof. Suppose that $f$ is $\mu$-integrable for each $\mu \in M(G, X)$. Take a semi-norm $u \in \mathcal{S}$ in $X$ and a number $\pi \in \mathbf{K}$ such that $0<|\pi|<1$. If $f$ is not bounded, then there exists a sequence $b_{j} \in \mathbf{G}$ such that $b_{i} \neq b_{j}$ for each $i \neq j$ and $\lim _{n \rightarrow \infty} u\left(\pi^{n} f\left(b_{n}\right)\right)=\infty$. Put $\mu:=\sum_{n} \pi^{n} x_{n} \delta_{b_{n}}$, where $\delta_{b}(A):=1$ if $b \in A, \delta_{b}(A)=0$ if $b \notin A, x_{n} \in X, u\left(x_{n}\right)=1$. Therefore, $\mu \in M(G, X)$ and $N_{\mu, u}\left(b_{n}\right)=|\pi|^{n}$ for every $n \in \mathbf{N}$, hence $\|f\|_{\mu, u}=\infty$ 
and $f \notin L(G, \operatorname{Bco}(G), \mu, X ; \mathrm{g})$. In view of Theorem 7.9 [37] if $V$ is a compact subset in $G$, then there exists a measure $\lambda: \operatorname{Bco}(G) \rightarrow \mathbf{K}$ such that $N_{\lambda}(x)=1$ for each $x \in V$ and $N_{\lambda}(x)=0$ for each $x \in G \backslash V$. Take $y \in X$ with $u(y)=1$, then $\mu=y \lambda$ is the $X$-valued measure and $N_{\mu, u}=C h_{V}$. Therefore, due to Lemma 54 and Theorem $\left.56 \mathrm{f}\right|_{V}$ is continuous.

Suppose now that $f$ is bounded and its restriction to each compact subset of $G$ is continuous. Take any $\mu \in M(G, X)$, then due to Corollary 55 the mapping $f$ is $\operatorname{Bco}(G)_{\mu}$-continuous. If $z \in \mathrm{g}$, then $q_{z} \in L(G, \operatorname{Bco}(G), \mu, X ; \mathrm{g})$, where $q_{z}(x)=z$ for every $x \in G$. Take $z \in$ g such that $u(f(x)) \leq u(z)$ for each $x \in G$, consequently, in view of Corollary $57 f \in L(G, \operatorname{Bco}(G), \mu, X ; \mathrm{g})$.

69. Definition. Let $G$ be a zero-dimensional Hausdorff topological space. If for each $U \subset G$ it is clopen in $G$ if and only if $U \cap V$ is clopen in $V$ for each compact subset $V$ in $G$, then $G$ is called the $k_{0}$-space.

70. Corollary. If $G$ is a $k_{0}$-space, then $B C(G, \mathrm{~g})=\bigcap_{\mu \in M(G, X)} L(G, \operatorname{Bco}(G), \mu, X ; \mathrm{g})$.

Proof. By Theorem 3.3.21 [10] a mapping $f: G \rightarrow Y$ of a $k$-space $G$ into a topological space $Y$ is continuous if and only if for each compact subset $V$ in $G$ the restriction $\left.f\right|_{V}$ of $f$ to $V$ is continuous. Therefore, due to Theorem 67 we get the statement of this corollary.

71. Definition. A functional $J \in B C(G, \mathrm{~g})^{*}$ is said to have a compact support, if there exists a compact subset $V$ in $G$ such that $J(f)=0$ for each $f \in B C(G, \mathrm{~g})$ with $f(x)=0$ for every $x \in V$.

A hood on $G$ is a mapping $h: G \rightarrow[0, \infty)$ such that the set $\{x \in G$ : $h(x) \geq \epsilon\}$ is compact for each $\epsilon>0$. A subset $W \subset B C(G, \mathrm{~g})$ is called strictly open if for each $f \in W$ and a semi-norm $u \in \mathcal{S}$ in $\mathrm{g}$ there exists a hood $h$ such that $W \supset\left\{g \in B C(G, \mathrm{~g}): \sup _{x \in G} u(f(x)-g(x)) h(x) \leq 1\right\}$. Strictly open subsets in $B C(G, \mathrm{~g})$ form a topology in $B C(G, \mathrm{~g})$ called the strict topology.

72. Theorem. The following conditions on $J \in B C\left(G, \operatorname{Mat}_{n}(\mathbf{K})\right)^{*}$ are equivalent:

(1) there exists $\mu \in M\left(G, \operatorname{Mat}_{n}(\mathbf{K})\right)$ such that $J=\lambda_{\mu}$ (see Theorem 66$)$;

(2) for each $\epsilon>0$ there exists a compact subset $V$ in $G$ such that $|J(f)| \leq$ $\max \left\{\|J\| \sup _{x \in V}\|f(x)\|, \epsilon\|f\|\right)$ for every $f \in B C\left(G, \operatorname{Mat}_{N}(\mathbf{K})\right)$;

(3) $J$ is the limit of elements in $B C\left(G, M_{n}(\mathbf{K})\right)^{*}$ having compact supports;

(4) $J$ is strictly continuous.

Proof. If $W_{1}$ and $W_{2}$ are strictly open, $f \in W=W_{1} \cap W_{2}, u \in \mathcal{S}$, then there exist hoods $h_{j}$ such that $W_{j} \supset\left\{g \in B C(G, \mathrm{~g}): \sup _{x \in G} u(f(x)-\right.$ 
$\left.g(x)) h_{j}(x) \leq 1\right\}$ for $j=1,2$, then $W \supset\{g \in B C(G, \mathrm{~g}): u(f(x)-g(x)) h(x) \leq$ $1\}$, where $h(x)=\max \left(h_{1}(x), h_{2}(x)\right)$ for each $x$ is the hood such that $\{x \in$ $G: h(x) \geq \epsilon\}=\left\{x \in G: h_{1}(x) \geq \epsilon\right\} \cup\left\{x \in G: h_{2}(x) \geq \epsilon\right\}$ is compact for each $\epsilon>0$ as the union of two compact sets. Thus strictly open subsets form a topology.

The $\mathbf{K}$-algebra $\operatorname{Mat}_{n}(\mathbf{K})$ is normed. Since $\operatorname{Mat}_{n}(\mathbf{K})$ is the finite dimensional space over $\mathbf{K}$ its topologically dual space is isomorphic with $\operatorname{Mat}_{n}(\mathbf{K})$. For a compact subset $W$ in $G$ let $R_{W}$ denotes the restriction mapping $R_{W}$ : $B C\left(G, M_{a t}(\mathbf{K})\right) \rightarrow C\left(W, \operatorname{Mat}_{n}(\mathbf{K})\right)$. In view of Theorem 5.24 [37] there exists a K-linear isometric embedding $T_{W}: C\left(W, \operatorname{Mat}_{n}(\mathbf{K})\right) \hookrightarrow P C\left(G, \operatorname{Mat}_{n}(\mathbf{K})\right)$ such that $R_{W} \circ T_{W}=I$, since $n \in \mathbf{N}$, where $P C(G, X)$ denotes the closed $\mathbf{K}$-linear hull in $B C(G, X)$ of the subset $\left\{C h_{A}: A \in \operatorname{Bco}(G), A\right.$ is compact $\}$.

If $J=\lambda_{\mu}$ with $\mu \in M\left(G, \operatorname{Mat}_{n}(\mathbf{K})\right)$, then $N_{\mu}$ is the hood and $|J(f)| \leq$ $\|f\|_{N_{\mu}}$ for every $f \in B C\left(G, \operatorname{Mat}_{n}(\mathbf{K})\right)$, consequently, $J$ is strictly continuous, that is, (1) $\Rightarrow(4)$.

If $J$ is strictly continuous take $\pi \in \mathbf{K}$ with $0<|\pi|<1$. There exists a hood $h$ for which $\left\{f:\|f\|_{h}<1\right\} \subset\{f:|J(f)| \leq|\pi|\}$. Therefore, $|J(f)| \leq\|f\|_{h}$ for each $f$. For $\epsilon>0$ put $W:=\{x \in G: h(x) \geq \epsilon\}$. If $f \in B C\left(G, \operatorname{Mat}_{n}(\mathbf{K})\right)$, then take $g:=T_{W} R_{W} f$, hence $J(f)=J(f-g)+J(g)$ and $|J(f-g)| \leq \sup _{x \in G}\|f(x)-g(x)\| h(x) \leq \sup _{x \in G \backslash W}\|f(x)-g(x)\| \epsilon \leq\|f\| \epsilon$ and $|J(g)| \leq\|J\|\|g\| \leq\|J\| \sup _{x \in W}\|f(x)\|$, consequently, (4) $\Rightarrow$ (3).

Suppose that (2) is satisfied, then $J_{W}$ has the compact support. Therefore, $\left|J(f)-J_{W}(f)\right|=\left|J\left(f-T_{W} R_{W} f\right)\right| \leq \epsilon\left\|f-T_{W} R_{W} f\right\|<\epsilon\|f\|$, consequently, $\left\|J-J_{W}\right\| \leq \epsilon$, hence $(2) \Rightarrow(3)$.

Let (3) be satisfied. We have that $M\left(G, \operatorname{Mat}_{n}(\mathbf{K})\right)$ is complete and $\lambda$ is the isometry, consequently, the range of $\lambda$ is closed in $B C\left(G, M_{n}(\mathbf{K})\right)^{*}$. Therefore, without loss of generality consider $J$ with the compact support. Suppose that $W \subset G$ and $J(f)$ for $f$ vanishing identically on $W$. Put $\mu_{i, j}^{J}(A):=J\left(E_{i, j} C h_{A}\right)$ for each $A \in \operatorname{Bco}(G)$ and all $i, j=1, \ldots, n$, hence $\mu: \operatorname{Bco}(G) \rightarrow \operatorname{Mat}_{n}(\mathbf{K})$ is additive, where $E_{i, j}$ is the matrix with the element 1 at the $(i, j)$-th place and zeros at others places, $\mu(A)=\mu^{J}(A)$ is the matrix with matrix elements $\mu_{i, j}^{J}(A)$. Then $N_{\mu}(x)=0$ for every $x \in G \backslash W$ and $N_{\mu}$ is bounded on $G$, consequently, $\mu$ is the measure on $\operatorname{Bco}(G)$.

The normed space $C\left(W, \operatorname{Mat}_{n}(\mathbf{K})\right)$ has an orthonormal base consisting of functions $E_{i, j} C h_{A}$, where $i, j=1, \ldots, n$ and $A \in \operatorname{Bco}(G)$. Suppose that $f \in B C\left(G, \operatorname{Mat}_{n}(\mathbf{K})\right)$, then there exists $A_{k} \in \operatorname{Bco}(G)$ and $b_{k} \in \operatorname{Mat}_{n}(\mathbf{K})$ such that $b_{k}=a_{k} E_{i(k), j(k)}$ and $\lim _{k \rightarrow \infty} a_{k}=0$ and $f=\sum_{k} b_{k} C h_{A_{k}}$ uni- 
formly on $W$. Therefore, $J(f)=J\left(\sum_{k} b_{k} C h_{A_{k}}\right)=\sum_{k} a_{k} \mu_{i(k), j(k)}\left(A_{k}\right)=$ $\int_{G} \sum_{k} a_{k} C h_{A_{k}} \mu_{i(k), j(k)}(d x)=\int_{G} \sum_{k} b_{k} \mu\left(A_{k}\right)=\int_{G} f(x) \mu(d x)$, since $\mu(A)=$ $\sum_{i, j=1}^{n} E_{i, j} J\left(E_{i, j} C h_{A}\right)$, consequently, (3) $\Rightarrow(1)$.

73. Corollary. Let $G$ and $H$ be zero-dimensional Hausdorff spaces, let also $X$ be a complete topological algebra over $\mathbf{K}$. If $\mu \in M(G, X)$ and $\nu \in M(H, X)$ are tight measures, then $\mu \times \nu$ is a tight measure on $G \times H$, $\mu \times \nu \in M(G \times H, X)$.

Proof. This follows from Theorem $72(2)$.

74. Example. Consider convolutions of tight measures. Suppose that $G$ is a zero-dimensional Hausdorff topological semi-group and $X$ be a topological algebra over $\mathbf{K}$. For $\mu, \nu \in M(G, X)$ and $f \in B C(G, \mathrm{~g})$ define $J f:=\int_{G} f(x y)(\mu(d x) \times \nu(d y))$. If $u \in \mathcal{S}$ is a consistent semi-norm in $X$ and $\mathrm{g}$, then $u(J f) \leq \sup _{x, y \in G} u(f(x y)) N_{\mu, u}(x) N_{\nu, u}(y)$. For each $\epsilon>0$ we have that $G_{\mu, u, \epsilon}:=\left\{x \in G: N_{\mu, u}(x) \geq \epsilon\right\}$ and $G_{\nu, u, \epsilon}:=\left\{y \in G: N_{\nu, u}(y) \geq \epsilon\right\}$ are compact, hence their product $G_{\mu, u, \epsilon} G_{\nu, u, \epsilon}$ is compact, moreover, $u(J f) \leq$ $\max \left\{\sup \left\{u(f(z)): z \in G_{\mu, u, \epsilon} G_{\nu, u, \epsilon}\right\}\|\mu\|_{u}\|\nu\|_{u} ;\|f\|_{u}\|\mu\|_{u} \epsilon ;\|f\|_{u}\|\nu\|_{u} \epsilon\right\}$. Therefore, $J$ is induced by a tight measure denoted by $\mu * \nu$ such that $\int_{G} f(x)[\mu *$ $\nu](d x)=\int_{G} f(x y)(\mu(d x) \times \nu(d y))$ for each $f \in B C(G, X)$. In particular, for $f=C h_{A}$ with $A \in \operatorname{Bco}(G)$ we get

(i) $[\mu * \nu](A)=(\mu \times \nu)(\{(x, y) \in G \times G, x y \in A\})$.

The tight measure $\mu * \nu$ is called the convolution product of $\mu$ and $\nu$. Evidently, $(a \mu+b \zeta) * \nu=(a \mu * \nu)+(b \zeta * \nu)$ and $\mu *(a \nu+b \zeta)=(a \mu * \nu)+(b \mu * \zeta)$ for each $a, b \in \mathbf{K}$ and $\mu, \nu, \zeta \in M(G, X)$, since $\mathbf{K}$ is the commutative field. Hence $M(G, X)$ is the algebra with the addition $(\mu+\nu)(A)=\mu(A)+\nu(A)$ for each $A \in \operatorname{Bco}(G)$ and the multiplication given by the convolution product of measures.

From $(i)$ it follows, that $N_{\mu * \nu, u}(z)=\sup _{x, y \in G, x y=z} N_{\mu, u}(x) N_{\nu, u}(y)<\infty$, consequently, $M(G, X)$ is the topological algebra with the family of seminorms $\|\mu\|_{u}:=\sup _{x \in G} N_{\mu, u}(x), u \in \mathcal{S}$, such that $\|\mu * \nu\|_{u} \leq\|\mu\|_{u}\|\nu\|_{u}$ for each $\mu, \nu \in M(G, X)$.

75. Lemma. The mapping $39(S I)$ and Conditions $35(M 1-M 4)$ induce an isometry between $L^{2}(\mathcal{R}(G), \mathrm{g})$ and $L^{2}(\xi, \mathrm{g})$.

Proof. At first demonstrate, that there exists a linear isometric mapping of $L^{0}(\mathcal{R}, \mathrm{g})$ on $L^{0}(\xi, \mathrm{g})$. Let $f(x)=\sum_{k} a_{k} C h_{A_{k}}(x)$ and $g(x)=\sum_{l} b_{l} C h_{A_{l}}(x)$ be simple functions in $L^{0}(\mathcal{R}, \mathrm{g})$, where $a_{k}, b_{l} \in \mathrm{g}$ (see Definition 39). Then due to Conditions $35(M 1-M 4)$ and $39(1-7)$ there are the equalities:

(1) $M\left[\left(\int_{G}[f(x) \xi(d x)),\left(\int_{G} g(x) \xi(d x)\right)\right]=\sum_{k}\left[a_{k}, b_{k}\right] \mu\left(A_{k}\right)\right.$ 
$=\int_{G}[f(x), g(x)] \mu(d x)$,

since $\mathrm{g}^{2} \ni\{a, b\} \mapsto[a, b] \in L c(\mathrm{~g})$ is the continuous mapping, $\mu(A) \in L c(X)$ for each $A \in \mathcal{R}$.

In view of Lemma 38 there exists the $\mathbf{K}$-valued measure $\operatorname{Tr} \mu$. Therefore,

(2) $M\left(\left(\int_{G} f(x) \xi(d x)\right),\left(\int_{G} g(x) \xi(d x)\right)\right)=\sum_{k} \operatorname{Tr}\left(b_{k}^{T} a_{k} \mu\right)\left(A_{k}\right)$

$=\int_{G} \operatorname{Tr}\left(g^{T}(x) f(x) \mu\right)(d x)$, since $\mathrm{g}^{2} \ni\{a, b\} \mapsto(a, b) \in \mathbf{K}$ is the continuous mapping from $\mathrm{g}^{2}$ into the field $\mathbf{K}$, where $(\operatorname{Tr} \mu)(A):=\operatorname{Tr} \mu(A)$ for each $A \in \mathcal{R}(G)$.

If $F_{1} \in \operatorname{Lin}(X)$ and $F_{2} \in L c(X)$, then $F_{1} F_{2} \in L c(X)$. To each $b \in \mathrm{g}$ there corresponds the $\mathbf{K}$-linear continuous operator $X \ni x \mapsto b x \in X$. Naturally $\operatorname{Lin}(X)$ and $\operatorname{Lc}(X)$ are the left g-modules, since $(b F) \in \operatorname{Lin}(X)$ for each $F \in \operatorname{Lin}(X)$ and $(b F) \in \operatorname{Lc}(X)$ for every $F \in L c(X)$ and each $b \in \mathrm{g}$, where $(b F)(x):=b(F x)$ for all $x \in X$. Since $\mu(A) \in L c(X)$ and $g^{T}(x) f(x) \in \mathrm{g}$, then $g^{T}(x) f(x) \mu(A) \in L c(X)$ for each $x \in G$ and $A \in \mathcal{R}(G)$ and all $f, g \in L^{0}(\mathcal{R}, \mathrm{g})$.

Take in $L^{0}(\mathcal{R}, \mathrm{g})$ the semi-norm

(3) $\|f\|_{2, \mu}=\left[\sup _{x \in G} N_{T r f^{T} f \mu}(x)\right]$

and in $L^{0}(\xi, \mathrm{g})$ put

(4) $\left\|\eta_{f}\right\|_{2, N_{P}}:=\left[\sup _{x \in G}|(f(x) \xi(x), f(x) \xi(x))| N_{P}(x)\right]^{1 / 2}$

for each $\eta=\int_{G} f(x) \xi(d x)$. The semi-norm (4) is continuous relative to the family of semi-norms $43(1)$.

Semi-norm (3) is continuous relative to the family of semi-norms:

(5) $\|f\|_{\mu, u}:=\sup _{x \in G} N_{f^{T} f \mu, u}(x)$.

Since $M((a \xi(A), b \xi(A)))=\operatorname{Tr}\left(b^{T} a \mu(A)\right)$ for each $A \in \mathcal{R}(G)$, then $N_{\operatorname{Tr} f^{T} f \mu}(x)=$ $\inf _{A \in \mathcal{R}(G), x \in A}\|A\|_{T r f^{T} f \mu}$, where $\left(f^{T} f \mu\right)(d x)=f^{T}(x) f(x) \mu(d x)$. At the same time $M(a \xi(B), b \xi(B))=\int_{\Omega}(a \xi(\omega, B), b \xi(\omega, B)) P(d \omega),|M(a \xi(B), b \xi(B))| \leq$ $\sup _{\omega \in \Omega}|(a \xi(\omega, B), b \xi(\omega, B))| N_{P}(\omega)$ for each $a, b \in \mathrm{g}$.

In view of Lemma $38 \operatorname{Trg}^{T} f \mu$ is the measure for each $f, g \in L^{0}(\mathcal{R}, \mathrm{g})$, consequently, taking a shrinking family $\mathcal{S}$ in $\mathcal{R}(G)$ such that $\bigcap_{A \in \mathcal{S}} A=\{x\}$ gives

$N_{\operatorname{Trg}^{T} f \mu}(x)=\inf _{A \in \mathcal{R}(G), x \in A}\left[\sup _{B \in \mathcal{R}(G), B \subset A_{k}, k} \sup _{\omega \in \Omega, k}\left|\left(a_{k} \xi(\omega, B), b_{k} \xi(\omega, B)\right)\right| N_{P}(\omega)\right]$. Thus $N_{T r g^{T} f \mu}(x)=\inf _{A \in \mathcal{R}(G), x \in A}\left[\sup _{B \in \mathcal{R}(G), B \subset A_{k}, k}\left\|\left(a_{k} \xi(*, B), b_{k} \xi(*, B)\right)\right\|_{L^{2}(P, \mathbf{K})}\right]$ and $\left.\left\|A_{k}\right\|_{T r g^{T} f \mu}=\left\|\left(a_{k} \xi\left(*, A_{k}\right), b_{k} \xi\left(*, A_{k}\right)\right)\right\|_{L^{2}(P)}\right]$ for each $k=1, \ldots, m$ due to Lemma 2 and due to the choice $\left\|A_{k}\right\|_{T r b_{k}^{T} a_{k} \mu}=\left|\operatorname{Tr} b_{k}^{T} a_{k} \mu\left(A_{k}\right)\right|$ without loss of generality.

On the other hand, $M[a \xi(A), b \xi(A)]=b^{T} a \mu(A) \in L c(X)$ for each $A \in$ 
$\mathcal{R}(G)$. Then $N_{b^{T} a \mu, u}(x)=\inf _{A \in \mathcal{R}(G), x \in A}\left[\sup _{B \in \mathcal{R}(G), B \subset A} u\left(b^{T} a \mu(B)\right)\right]$

$\leq u\left(b^{T} a\right) N_{\mu, u}(x)<\infty$, where $N_{\mu, u}(x):=\inf _{A \in \mathcal{R}(G), x \in A}\left[\sup _{B \in \mathcal{R}(G), B \subset A} u(\mu(B))\right.$.

Therefore, $\|f\|_{\mu, u}=\|f\|_{2, P, u}$ for each $f \in L^{0}(\mathcal{R}, \mathrm{g})$ and each consistent seminorm $u$ in $\mathrm{g}, X$ and $\operatorname{Lin}(X)$.

The mapping $\psi$ from $39(S I)$ also is $\mathbf{K}$-linear from $L^{0}(\xi, \mathrm{g})$ into $L^{0}(\mathcal{R}(G), \mathrm{g})$ such that $\psi$ is the isometry relative to the consistent semi-norms 43(1) and 75(5) due to Formula (1) and Lemmas 2, 45 and Theorem 56.

Two spaces $L^{2}(P, \mathrm{~g})$ and $L^{2}(\mu, \mathrm{g})$ are complete by their definitions, consequently, $\psi$ has the $\mathbf{K}$-linear extension from $L^{2}(\mathcal{R}(G), \mathrm{g})$ onto $L^{2}(\xi, \mathrm{g})$ which is the isometry between $L^{2}(\mathcal{R}(G), \mathrm{g})$ and $L^{2}(\xi, \mathrm{g})$.

76. Definition. If $f \in L^{2}(\mathcal{R}(G), \mathrm{g})$, then put by the definition:

$\eta=\psi(f)=\int_{G} f(x) \xi(d x)$.

The random vector $\eta$ we call the non-archimedean stochastic integral of the function $f$ by an orthogonal stochastic measure $\xi$.

77. Remark. Consider random vectors of the form:

$\eta(t)=\int_{G} g(t, x) \xi(d x)$, where $\xi$ is an orthogonal stochastic measure on a measurable space $(G, \mathcal{R}(G))$ with values in $X$ and a structural measure $\mu$ with values in $L c(X)$ as above, $t \in T, g(t, x) \in L^{2}(G, \mathcal{R}(G), \mu, \mathrm{g})$ as the function by $x \in G$ for each $t \in T$, where $T$ is a set.

The covariance operator of a random vector $\eta$ is

(1) $B\left(t_{1}, t_{2}\right)=M\left\{\eta^{T}\left(t_{1}\right), \eta\left(t_{2}\right)\right\}=\int_{G}\left\{g^{T}\left(t_{1}, x\right), g\left(t_{2}, x\right)\right\} \mu(d x)$, moreover,

(2) $M\left\{\eta\left(t_{1}\right), \eta^{T}\left(t_{2}\right)\right\}=\int_{G}\left\{g\left(t_{1}, x\right), g^{T}\left(t_{2}, x\right)\right\} \operatorname{Tr} \mu(d x)$

in the notation of $\S \S 31,39$, where $X$ is the left g-module, while $L c(X)$ is supplied with the natural structure of the left $\operatorname{Lc}(\mathrm{g})$-module, $\left\{a^{T}, b\right\} \in$ $\operatorname{Lin}(\mathrm{g}),\left\{a, b^{T}\right\} \in \mathrm{g}$ for each $a, b \in \mathrm{g}$. Denote by $L^{2}\{g\}$ the closure in $L^{2}(G, \mathcal{R}(G), \mu, \mathrm{g})$ of the $\mathbf{K}$-linear span of the family of functions $\{g(t, x): t \in$ $T\}$. Therefore, $L^{2}\{g\}$ is the $\mathbf{K}$-linear closed subspace in $L^{2}(G, \mathcal{R}(G), \mu, \mathbf{g})$. If $L^{2}\{g\}=L^{2}(G, \mathcal{R}(G), \mu, \mathrm{g})$, then the system of functions $\{g(t, x): t \in T\}$ is called complete in $L^{2}(G, \mathcal{R}(G), \mu, \mathrm{g})$.

Let $\{\eta(t): t \in T\}$ be a $X$-valued random vector. Denote by $L^{0}\{\eta\}$ the family of all random vectors of the form:

$\zeta=\sum_{k=1}^{l} a_{k} \eta\left(t_{k}\right)$, where $l \in \mathbf{N}, t_{k} \in T, a_{k} \in \mathrm{g}$. Then $L^{2}\{\eta\}$ denotes the closure of $L^{0}\{\eta\}$ in $L^{2}(\Omega, \mathcal{R}, P, X)$.

A family of random vectors $\left\{\zeta_{\beta}: \zeta_{\beta} \in L^{2}(\Omega, \mathcal{R}, P, X) ; \beta \in \Lambda\right\}$ is called subordinated to the random $X$-valued function $\{\eta(t): t \in T\}$, if $\zeta_{\beta} \in L^{2}\{\eta\}$ for each $\beta \in \Lambda$. 
78. Lemma. If $X$ is a left $\mathrm{g}$-module, then $\operatorname{Lin}(X)$ is a left Lin $(\mathrm{g})$ module, $L c(X)$ is a left Lin $(\mathrm{g})$-module as well as left $L c(\mathrm{~g})$-module.

Proof. Let $x \in X, y \in \mathrm{g}, A \in \operatorname{Lin}(\mathrm{g})$ and $B \in \operatorname{Lin}(X)$, then $B x \in$ $X, y(B x) \in X$ and $A y \in \mathrm{g}$, hence $A y B x \in X$, since the left module is associative. Particularly for $1 \in \mathrm{g}$ it gives $A B \in \operatorname{Lin}(X)$. Therefore, there exists the multiplication $\operatorname{Lin}(\mathrm{g}) \times \operatorname{Lin}(X) \ni(A, B) \mapsto A B \in \operatorname{Lin}(X)$. If $B \in L c(X)$, then $A y B \in L c(X)$, since $v B \in L c(X)$ for each $v \in \mathrm{g}$. In particular, for $y=1$, consequently, $\operatorname{Lc}(X)$ is the left $\operatorname{Lin}(\mathrm{g})$-module and inevitably left $L c(\mathrm{~g})$-module, since $L c(\mathrm{~g}) \subset \operatorname{Lin}(\mathrm{g})$.

79. Theorem. Let a covariance operator $B\left(t_{1}, t_{2}\right)$ of a random $X$-valued function $\{\eta(t): t \in T\}$ admits representation $76(1)$, where $X$ and $\mathrm{g}$ are as in $\S \S 30$ and $39, \mu$ is a $L c(X)$-valued measure on $(G, \mathcal{R}(G)), \mu^{T}=\mu, g(t, x) \in$ $L^{2}(G, \mathcal{R}(G), \mu, L c(X) ; \mathrm{g})$ for each $t \in T$ and the family $\{g(t, x): t \in T ;\}$ is complete in $L^{2}(G, \mathcal{R}(G), \mu, L c(X) ; \mathrm{g})$. Then $\eta(t)$ can be presented in the form:

(1) $\eta(t)=\int_{G} g(t, x) \xi(d x)$

with probability 1 for each $t \in T$, where $\xi$ is a stochastic orthogonal $X$ valued measure subordinated to the random function $\eta(t)$ and with a structure function $\mu$.

Proof. Consider functions of the form:

(2) $f(x)=\sum_{k=1}^{l} b_{k} g\left(t_{k}, x\right)$, where $t_{k} \in T, b_{k} \in \mathrm{g}, l \in \mathbf{N}$. Put

(3) $\psi(f)=\zeta=\sum_{k=1}^{l} b_{k} \eta\left(t_{k}\right)$. Denote by $L^{0}\{g\}$ the family of all vectors of Form (2). In $L^{0}\{g\}$ there is the $\mathbf{K}$-bi-linear functional:

(4) $\left(f_{1}, f_{2}\right):=\int_{G}\left\{f_{1}(x), f_{2}^{T}(x)\right\} \operatorname{Tr} \mu(d x)$.

In view of Lemma 75 the mapping $\zeta=\psi(f)$ is the $\mathbf{K}$-linear topological isomorphism of $L^{0}\{g\}$ onto $L^{0}\{\eta\}$. When particularly $X$ and $\mathrm{g}$ are normed, then $\psi$ is the isometry. Thus $\psi$ has the continuous extension up to the K-linear topological isomorphism of $L^{2}\{g\}$ onto $L^{2}\{\eta\}$.

If $A \in \mathcal{R}(G)$, then $C h_{A} \in L^{2}(G, \mathcal{R}(G), \mu, \mathrm{g})$, since $1 \in \mathrm{g}$. But $L^{2}\{g\}=$ $L^{2}(G, \mathcal{R}(G), \mu, \mathrm{g})$ due to completeness of the family $\{g(t, x): t \in T\}$. Therefore, $C h_{A} \in L^{2}(G, \mathcal{R}(G), \mu, \mathrm{g})$. Put $\xi(A):=\psi\left(C h_{A}\right)$, then $\xi(A)$ is the orthogonal stochastic measure with the structure function $\mu$ due to Lemma 78, since (5) $M\left\{\xi^{T}(A), \xi(B)\right\}=\int_{G}\left\{C h_{A}^{T}(x), C h_{B}(x)\right\} \mu(d x)=\mu(A \cap B)$ for each $A, B \in \mathcal{R}(G)$.

Let now $\gamma(t):=\int_{G} g(t, x) \xi(d x)$. Since $M\left\{\eta^{T}(t), \xi(A)\right\}=\int_{G}\left\{g^{T}(t, x), C h_{A}(x)\right\} \mu(d x)$ and $M\left\{\xi^{T}(A), \eta(t)\right\}=\int_{G}\left\{C h_{A}^{T}(x), g(t, x)\right\} \mu(d x)$ and $\psi$ is the $\mathbf{K}$-linear topo- 
logical isomorphism, then $M\left\{\eta^{T}(t), \gamma(t)\right\}=M\left\{\gamma^{T}(t), \eta(t)\right\}=\int_{G}\left\{g^{T}(t, x), g(t, x)\right\} \mu(d x)$. Therefore, $M\left\{C h_{A}(\eta(t)-\gamma(t))^{T}, C h_{A}(\eta(t)-\gamma(t))\right\}=M\left\{C h_{A} \eta^{T}(t), C h_{A} \eta(t)\right\}-$ $M\left\{C h_{A} \eta^{T}, C h_{A} \gamma(t)\right\}-M\left\{C h_{A} \gamma^{T}(t), C h_{A} \eta(t)\right\}+M\left\{C h_{A} \gamma^{T}(t), C h_{A} \gamma(t)\right\}=$ 0 for each $A \in \mathcal{R}(G)$, consequently, 79(1) is accomplished with probability 1 for each $t \in T$.

80. Definition. Let $\eta(t)$ be a g-valued stochastic process or stochastic function such that for each $n \in \mathbf{N}$ and each $t_{1}, \ldots, t_{n} \in \mathbf{T}$ with $t, t+t_{1}, \ldots, t+$ $t_{n} \in \mathbf{T}$ the mutual distribution of $\eta\left(t+t_{1}\right), \ldots, \eta\left(t+t_{n}\right)$ is independent from $t$, where $\mathbf{T}$ is an additive semigroup. Then $\eta(t)$ is called the stationary stochastic function, where $P: \mathcal{R} \rightarrow \mathbf{K}$ is a probability measure.

Suppose that $\mathbf{T}$ is a uniform space. A g-valued stochastic function $\eta(t) \in$ $L^{b}(\Omega, \mathcal{R}, P, \mathbf{K} ; \mathrm{g}), t \in \mathbf{T}, 1 \leq b<\infty$, is called mean- $b$-continuous at $t_{0} \in \mathbf{T}$, if there exists $\lim _{t \rightarrow t_{0}} \eta(t)=\eta\left(t_{0}\right)$ in the sense of convergence in the space $L^{b}(\Omega, \mathcal{R}, P, \mathbf{K} ; \mathrm{g})$, when $t$ tends to $t_{0}$ in $\mathbf{T}$. In particular, for $b=2$ it is meansquare continuity and convergence respectively. If $\eta(t)$ is mean- $b$-continuous at each point of $\mathbf{T}$, then $\eta(t)$ is called mean- $b$-continuous on $\mathbf{T}$.

81. Suppose that an algebra $\mathbf{g}$ over $\mathbf{C}_{\mathbf{p}}$ has a uniformity $\tau$ relative to which it is complete. Let $\mathbf{g}$ has a $\mathbf{Q}_{\mathbf{p}}$-linear embedding into $c_{0}\left(\gamma, \mathbf{Q}_{\mathbf{p}}\right)$ for some set $\gamma$ such that the norm uniformity $n_{u}$ in $g$ inherited from the Banach space $c_{0}\left(\gamma, \mathbf{Q}_{\mathbf{p}}\right)$ with the standard norm $\|*\|$ is not stronger, than $\tau$, that is $n_{u} \subset \tau$, moreover, $\mathrm{g}$ is everywhere dense in $\left(c_{0}\left(\gamma, \mathbf{Q}_{\mathbf{p}}\right),\|*\|\right)$.

Theorem. Let $\eta(t)$ be a stationary mean-square-continuous stochastic process with values in $\mathrm{g}, t \in \mathbf{T}=\mathbf{C}_{\mathbf{r}}$, where $r$ and $p$ are mutually prime numbers, $M \eta(t)=0$, then there exists an orthogonal g-valued stochastic measure $\xi(A)$ on $\mathrm{Bco}\left(\mathbf{C}_{\mathbf{r}}\right)$ subordinated to $\eta(t)$ such that

(1) $\eta(t)=\int_{\mathbf{C}_{\mathbf{r}}} g(t, x) \xi(d x)$,

where $g(t, x)$ is a $\mathbf{C}_{\mathbf{p}}$-valued character from the additive group $\left(\mathbf{C}_{\mathbf{r}},+\right)$ into the multiplicative group $\left(\mathbf{C}_{\mathbf{p}}, \times\right)$. Between $L^{2}\{\eta\}$ and $L^{2}\{\mu\}$ there exists a $\mathbf{K}$-linear topological isomorphism $\psi$ such that

(2) $\psi(\eta(t))=g(t, *), \psi(\xi(A))=C h_{A}$, if

(3) $\zeta_{j}=\psi\left(f_{j}\right)$, then $\zeta_{j}=\int_{\mathbf{C}_{\mathbf{r}}} f_{j}(x) \xi(d x)$ and $M\left\{\zeta_{1}^{T}, \zeta_{2}\right\}=\int_{\mathbf{C}_{\mathbf{r}}}\left\{f_{1}(x)^{T}, f_{2}(x)\right\} \mu(d x)$.

82. Definition. Formula 81(1) is called the spectral decomposition of the stationary stochastic process. A measure $\xi(A)$ is called a stochastic spectral measure of the stationary stochastic process $\eta(t)$.

Proof of Theorem 81. Since $\eta(t)$ is a stationary stochastic process, then for each continuous function $f: \mathrm{g}^{n} \rightarrow \mathbf{K}$ the mean value $M f(\eta(t+$ $\left.\left.t_{1}\right), \ldots, \eta\left(t+t_{n}\right)\right)$ is independent from $t$, where $n \in \mathbf{N}$. By the condition of 
this theorem $\eta(t) \in L^{2}\left(\Omega, \mathcal{R}, P, \mathbf{C}_{\mathbf{p}} ; \mathbf{g}\right)$, consequently, there exist $M \eta(t)=m$ and

(4) $M\left\{[\xi(t)-m]^{T},[\xi(q)-m]\right\}=B(t-q) \in L c(\mathrm{~g})$ for each $t, q \in \mathbf{C}_{\mathbf{r}}$, where $m=0$. Evidently $B^{T}(t-q)=B(q-t)$ for each $t, q \in \mathbf{C}_{\mathbf{r}}$.

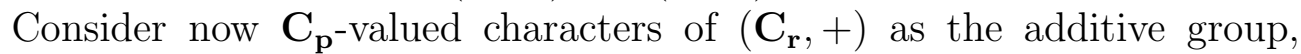
where $r=p^{\prime}, p \neq p^{\prime}$ are prime numbers. For $p$-adic numbers $x=\sum_{k=N}^{\infty} x_{k} p^{k}$, where $x \in \mathbf{Q}_{\mathbf{p}}, x_{k} \in\{0,1, \ldots, p-1\}, N \in \mathbf{Z}, N=N(x), x_{N} \neq 0, x_{j}=0$ for each $j<N$, put as usually $\operatorname{ord}_{p}(x)=N$ for the order of $x$, thus its norm is $|x|_{\mathbf{Q}_{\mathbf{p}}}=p^{-N}$. Define the function $[x]_{\mathbf{Q}_{\mathbf{p}}}:=\sum_{k=N}^{-1} x_{k} p^{k}$ for $N<0,[x]_{\mathbf{Q}_{\mathbf{p}}}=0$ for $N \geq 0$ on $\mathbf{Q}_{\mathbf{p}}$. Therefore, the function $[x]_{\mathbf{Q}_{\mathbf{p}}}$ on $\mathbf{Q}_{\mathbf{p}}$ is considered with values in the segment $[0,1] \subset \mathbf{R}$.

Consider the field $\mathbf{C}_{\mathbf{r}}$ as the vector space over the field $\mathbf{Q}_{\mathbf{r}}$. There is a multiplicative non-archimedean norm $|*|_{\mathbf{C}_{\mathbf{r}}}=|*|$ in $\mathbf{C}_{\mathbf{r}}$, which gives the uniformity in it. Take an equivalent uniformity given by a norm $|*|_{r}$ such that $|x|_{r} \in\left\{r^{l}: l \in \mathbf{Z}\right\} \cup\{0\}$ for each $x \in \mathbf{C}_{\mathbf{r}}$. If $x \neq 0$ put $|x|_{r}:=\min \left\{r^{l}:\right.$ $\left.|x|_{\mathbf{C}_{\mathbf{r}}} \leq r^{l}, l \in \mathbf{Z}\right\},|0|_{r}=0$, hence

(i) $|x|_{r} / r \leq|x|_{\mathbf{C}_{\mathbf{r}}} \leq|x|_{r}$ for each $x \in \mathbf{C}_{\mathbf{r}}$

and inevitably $\mathbf{C}_{\mathbf{r}}$ is the topological vector space over $\mathbf{Q}_{\mathbf{r}}$ relative to $|x|_{r}$. Since $\mathbf{C}_{\mathbf{r}}$ is the extension of $\mathbf{Q}_{\mathbf{r}}$, then the restriction of $|*|_{r}$ on $\mathbf{Q}_{\mathbf{r}}$ is the $r$-adic norm. On the entire $\mathbf{C}_{\mathbf{r}}$ this $|*|_{r}$ in general need not be multiplicative. Verify, that it is indeed a non-archimedean norm. At first $|x|_{r} \geq 0$ for each $x \in \mathbf{C}_{\mathbf{r}}$, $|x|_{r}=0$ if and only if $x=0$ due to $(i)$. If $x, y \in \mathbf{C}_{\mathbf{r}}, x \neq 0, y \neq 0$, then $|x|=r^{a},|y|=r^{b},|x+y|=r^{c}$ with $a, b, c \in \mathbf{R}, c \leq \max (a, b)$, where $r \geq 2$ is a prime number. Then $|x|_{r}=r^{A},|y|_{r}=r^{B},|x+y|_{r}=r^{C}$, where $a \leq A$, $b \leq B, c \leq C, A, B, C \in \mathbf{Z}$ are the least integers satisfying these inequalities. Therefore, $C \leq \max (A, B)$, consequently, $|x+y|_{r} \leq \max \left(|x|_{r},|y|_{r}\right)$ for each $x, y \in \mathbf{C}_{\mathbf{r}}$.

In view of Theorems 5.13 and $5.16[37]$ the $\mathbf{Q}_{\mathbf{r}}$-linear space $\left(\mathbf{C}_{\mathbf{r}},|*|_{r}\right)$ is isomorphic with $c_{0}\left(\alpha, \mathbf{Q}_{\mathbf{r}}\right)$, where $\alpha$ is a set, which is convenient to consider as an ordinal due to Zermelo theorem [10].

Let $(x, y):=(x, y)_{\mathbf{Q}_{\mathbf{r}}}:=\sum_{j \in \alpha} x_{j} y_{j}$ for $x, y \in \mathbf{C}_{\mathbf{r}}, x=\left(x_{j}: j \in \alpha, x_{j} \in\right.$ $\mathbf{Q}_{\mathbf{r}}$ ). This series $(x, y)$ converges in $\mathbf{Q}_{\mathbf{r}}$, since for each $\epsilon>0$ the set $\{j$ : $\left.\left|x_{j}\right|_{r} \geq \epsilon\right\}$ is finite.

If $X$ is a complete locally $\mathbf{C}_{\mathbf{r}}$-convex space, then it is the projective limit of Banach spaces $V_{u}:=X / Y_{u}$ over $\mathbf{C}_{\mathbf{r}}$, where $Y_{u}:=\{x \in X: u(x)=0\}$, $u$ is a semi-norm in $X, u \in \mathcal{S}$ [33]. Each $V_{u}$ can be supplied with the structure of a Banach space over $\mathbf{Q}_{\mathbf{r}}$. Therefore, $X$ can be supplied with the structure 
$X_{r}$ of the complete locally $\mathbf{Q}_{\mathbf{r}}$-convex space with a topology $\tau_{r}$.

Consider the case of such $X$, when $X_{r}$ has an embedding into $c_{0}\left(\beta, \mathbf{Q}_{\mathbf{r}}\right)$ for some $\beta \geq \alpha$ and the norm topology $n_{r}$ of $|*|_{r}$ in $X_{r}$ inherited from $c_{0}\left(\beta, \mathbf{Q}_{\mathbf{r}}\right)$ is such that $\tau_{r} \supset n_{r}$. Then each $\mathbf{Q}_{\mathbf{r}}$-linear continuous functional on $\left(c_{0}\left(\beta, \mathbf{C}_{\mathbf{r}}\right),|*|_{r}\right)$ is also continuous on $\left(X_{r}, \tau_{r}\right)$.

Define also a character with values in $\mathbf{C}_{\mathbf{p}}$ for $(X,+)$ as the additive group, $r \neq p$. Put

$\chi_{r, p ; s}(x)=\epsilon^{\left[(s, z)_{\mathbf{Q}_{\mathbf{r}}}\right]_{\mathbf{r}} / z}$, where $\epsilon=1^{z}$ is a root of unity in $\mathbf{C}_{\mathbf{p}}, z=$ $r^{\text {ord }} d_{r}\left[(s, z) \mathbf{Q}_{\mathbf{r}}\right] \mathbf{Q}_{\mathbf{r}}, s, z \in X_{r}$ or we can consider $s, z$ as elements in $X$ as well (see above).

For a tight measure $\mu: \mathcal{R}(X) \rightarrow L c(\mathrm{~g})$ or $\mu: \mathcal{R}(X) \rightarrow \mathbf{C}_{\mathbf{p}}$ the characteristic functional $\hat{\mu}$ is given by the formula: $\hat{\mu}(s):=\int_{X} \chi_{r, p ; s}(z) \mu(d z)$, where $s \in X_{r}, X$ is over $\mathbf{C}_{\mathbf{r}}$.

In general the characteristic functional of the measure $\mu: \mathcal{R}(G) \rightarrow L c(\mathrm{~g})$ or $\mu: \mathcal{R}(G) \rightarrow \mathbf{C}_{\mathbf{p}}$ is defined in the space $C^{0}\left(G, \mathbf{C}_{\mathbf{r}}\right)$ of continuous functions $f: G \rightarrow \mathbf{C}_{\mathbf{r}}$

$\hat{\mu}(f):=\int_{G} \chi_{r, p ; 1}(f(z)) \mu(d z)$, where $1 \in \mathbf{C}_{\mathbf{r}}, G$ is a totally disconnected topological Hausdorff space with a covering ring $\mathcal{R}(G)$.

In view of Theorems 2.21 and 2.30 [31] and Theorem 64 and (4) above there exists a $L c(\mathrm{~g})$-valued measure $\mu$ on $\mathrm{Bco}\left(\mathbf{C}_{\mathbf{r}}\right)$ such that $B(t)=\int_{\mathbf{C}_{\mathbf{r}}} \chi_{r, p ; 1}(t y) \mu(d y)$. Functions $g(t, y):=\chi_{r, p ; 1}(t y)$ are continuous and uniformly bounded. Since $|g(t, y)|_{C_{p}}=1$ for each $t, y \in \mathbf{C}_{\mathbf{r}}$, then $g(t, y) \in L^{2}\{\mu\}$.

In view of the Kaplansky Theorem A.4 [38] and Theorem 56 above the family of functions $\left\{g(t, y): t, y \in \mathbf{C}_{\mathbf{r}}\right\}$ is complete in $L^{2}\left(\mathrm{~g}, \mathrm{Bco}(\mathrm{g}), \mu, L c(\mathrm{~g}) ; \mathbf{C}_{\mathbf{p}}\right)$. Thus statements $81(1-3)$ follow from Theorem 79 . 


\section{References}

[1] M. Aigner. "Combinatorial theory". Springer-Verlag, Moscow (1979).

[2] S. Albeverio, W. Karwoski. "Diffusion on p-adic numbers". In: K. Ito, T. Hida (Editors). "Gaussian random fields". Nagoya 1990. World Scientific, River Edge, NJ (1991).

[3] I.Ya. Aref'eva, B. Dragovich, I.V. Volovich. "On the $p$-adic summability of the anharmonic oscillator"// Phys. Lett. V. B200, P. 512-514 (1988).

[4] A.N. Bikulov, I.V. Volovich. "p-adic Brounian motion"// Izv. Akad. Nauk. Ser. Mathem. V. 61: 3, P. 75-90 (1997).

[5] N. Bourbaki. "Integration. Vector integration. Haar measure. Convolution and representations". Nauka, Moscow (1970).

[6] C. Castro. "Fractals, strings as an alternative justification for El Naschie's cantorian spacetime and the fine structurte constants" // Chaos, Solitons and Fractals. V. 14, P. 1341-1351 (2002).

[7] Yu.L. Dalecky, S.V. Fomin. "Measures and differential equations in infinite-dimensional spaces". Kluwer Academic Publishers, Dordrecht (1991).

[8] B. Diarra. "Ultraproduits ultrametriques de corps values"// Ann. Sci. Univ. Clermont II. Sér. Math. V. 22, P. 1-37 (1984).

[9] G.S. Djordević, B. Dragovich. "p-adic and adelic harmonic oscillator with a time-dependent frequency"// Theor. and Math. Phys. V. 124: 2, P. 1059-1067 (2000).

[10] R. Engelking. "General topology". Mir, Moscow (1986).

[11] A. Escassut. "Analytic elements in p-adic analysis". World sceintific, Singapore (1995).

[12] S.N. Evans. "Continuity properties of Gaussian stochastic processes indexed by a local field" // Proceedings London Mathematical Society, Series 3, V. 56, P. 380-416 (1988). 
[13] S.N. Evans. "Local field Gaussian measures" // In: E. Cinlar, et.al. (Editors) "Seminar on Stochastic Processes 1988". P. 121-160. Birkhäuser, Boston (1989).

[14] S.N. Evans. "Equivalence and perpendicularity of local field Gaussian measures" // In: E. Cinlar, et.al. (Editors) "Seminar on Stochastic Processes 1990". P. 173-181. Birkhäuser, Boston (1991).

[15] S.N. Evans. "Local field Brownian motion"// J. Theoret. Probab. V. 6, P. 817-850 (1993).

[16] W. Feller. "An introduction to probability theory and its applications". V.V. 1, 2. John Wiley and Sons, Inc., New York (1966).

[17] I.I. Gihman, A.V. Skorohod. "Introduction in the theory or random processes". Nauka, Moscow (1977).

[18] P. Hennequin, A. Tortrat. "Probability theory and some its applications". Nauka, Moscow (1974).

[19] Y. Jang. "Non-archimedean quantum mechanics". Tohoku Math. Publ. V. 10 (1998).

[20] A. Khrennikov. "Interpretations of probability". VSP, Utrecht, 1999.

[21] A. Khrennikov, S.V. Kozyrev. "Ultrametric random field. Infinite Dimensional Analysis, Quantum Probability and Related Topics". V. 9: 2, P. 199-213 (2006).

[22] N. Koblitz. "p-adic numbers, $p$-adic analysis and zeta functions". Springer-Verlag, New York, 1977.

[23] A.N. Kochubei. "Pseudo-differential equations and stochastics over non-archimedean fields". Monogr. Textbooks Pure Appl. Math. V. 244. Marcel Dekker, Inc., New York (2001).

[24] A.N. Kochubei. "Limit theorems for sums of $p$-adic random variables"// Expo. Math. V. 16, P. 425-440 (1998). 
[25] S.V. Ludkovsky. "Topological groups of transformations of manifolds over non-archimedean fields, their representations and quasi-invariant measures. I, II". Sovrem. Mathem. and its Applications. V. 39, 2006; Sovrem. Mathem. Fundam. Napravl. V. 18, P. 5-100 (2006).

[26] S.V. Ludkovsky. "Stochastic processes on groups of diffeomorphisms and loops of real, complex and non-archimedean manifolds" // Fundam. i Prikl. Mathem. V. 7: 4, P. 1091-1105 (2001).

[27] S.V. Ludkovsky. "Stochastic processes on non-Archimedean Banach spaces"// Int. J. of Math. and Math. Sci. V. 2003: 21, P. 1341-1363 (2003).

[28] S.V. Ludkovsky. "Stochastic processes on totally disconnected topological groups"// Int. J. of Math. and Math. Sci. V. 2003: 48, P. 3067-3089 (2003).

[29] S.V. Ludkovsky. "Stochastic processes and antiderivational equations on non-Archimedean manifolds"// Int. J. of Math. and Math. Sci. V. 31: 1, P. 1633-1651 (2004).

[30] S.V. Ludkovsky. "Non-Archimedean valued quasi-invariant descending at infinity measures" // Int. J. of Math. and Math. Sci. V. 2005: 23, P. 3799-3817 (2005).

[31] Ludkovsky S.V. Quasi-invariant and pseudo-differentiable measures on non-Archimedean Banach spaces with values in non-Archimedean fields // J. Math. Sci. 2004. V. 122: 1. P. 2949-2983 (previous variant: Los Alamos preprint math.GM/0106170).

[32] S. Ludkovsky, A. Khrennikov. "Stochastic processes on nonArchimedean spaces with values in non-Archimedean fields" // Markov Processes and Related Fields. V. 9: 1, P. 131-162 (2003).

[33] L. Narici, E. Beckenstein. "Topological vector spaces". Marcel Dekker Inc., New York (1985).

[34] K.R. Partasarathy, R. Rao Ranga, S.R.S Varadhan. "Probability distributions on locally compact abelian groups"// Illinois J. Math. V. 7, P. 337-369 (1963). 
[35] K.R. Partasarathy. "Probability measures on metric spaces" // Probab. Math. Statist. V. 3. Academic Press, Inc., New York (1967).

[36] M. Reed, B. Simon. "Methods of Modern Mathematical Physics". V. 1. "Functional Analysis". Academic Press, New York (1977).

[37] A.C.M. van Rooij. "Non-Archimedean functional analysis". Marcel Dekker Inc., New York (1978).

[38] W.H. Schikhof. "Ultrametric calculus". Cambidge University Press, Cambridge (1984).

[39] A.N. Shirjaev. "Probability". Nauka, Moscow (1989).

[40] N.N. Vahanija, V.I. Tarieladze, S.A. Chobanjan. "Probability distributions in Banach spaces". Nauka, Moscow (1985).

[41] V.S. Vladimirov, I.V. Volovich, E.I. Zelenov. "p-adic analysis and mathematical physics". Fiz.-Mat. Lit., Moscow (1994).

[42] A. Weil. "Basic number theory". Springer, Berlin (1973).

[43] K. Yasuda. "Semi-stable processes on local fields" // Tohoku Math. J. V. 58, P. 419-431 (2006). 